Michael Tsamparlis • Andronikos Paliathanasis

\title{
The generic model of general relativity
}

Received: 10 December 2018 / Accepted: 10 January 2019 / Published online: 4 March 2019

(C) The Author(s) 2019

\begin{abstract}
We develop a generic spacetime model in general relativity which can be used to build any gravitational model within general relativity. The generic model uses two types of assumptions: (a) geometric assumptions in addition to the inherent geometric identities of the Riemannian geometry of spacetime and (b) assumptions defining a class of observers by means of their four-velocity $u^{a}$ which is a unit timelike vector field. The geometric assumptions as a rule concern symmetry assumptions (the so called collineations). The latter introduces the $1+3$ decomposition of tensor fields in spacetime. The $1+3$ decomposition results in two major results. The $1+3$ decomposition of $u_{a ; b}$ defines the kinematic variables of the model (expansion, rotation, shear and four-acceleration) and defines the kinematics of the gravitational model. The $1+3$ decomposition of the energy momentum tensor representing all gravitating matter introduces the dynamic variables of the model (energy density, the isotropic pressure, the momentum transfer or heat flux vector and the traceless tensor of the anisotropic pressure) as measured by the defined observers and defines the dynamics of the model. The symmetries assumed by the model act as constraints on both the kinematical and the dynamical variables of the model. As a second further development of the generic model we assume that in addition to the four-velocity of the observers $u_{a}$ there exists a second universal vector field $n_{a}$ in spacetime so that one has a so-called double congruence $\left(u_{a}, n_{a}\right)$ which can be used to define the $1+1+2$ decomposition of tensor fields. The $1+1+2$ decomposition leads to an extended kinematics concerning both fields building the double congruence and to a finer dynamics involving more physical variables. After presenting and discussing the results in their full generality we show how they are applied in practice by considering in a step by step approach the case of a string fluid in Bianchi I spacetime for the comoving observers.
\end{abstract}

Mathematics Subject Classification $\quad 83 \mathrm{C} 15 \cdot 83 \mathrm{C} 20 \cdot 17 \mathrm{~B} 66$

\section{Introduction}

General relativity (GR) is the first theory of Physics which uses geometry to such a great extent [6-9]. Newtonian Physics is also a geometric theory of Physics but the difference is that it uses the 3-D Euclidian geometry which

M. Tsamparlis (凶)

Faculty of Physics, Department of Astronomy-Astrophysics-Mechanics, University of Athens, Panepistemiopolis, 15783 Athens, Greece

E-mail: mtsampa@phys.uoa.gr

A. Paliathanasis

Instituto de Ciencias Físicas y Matemáticas, Universidad Austral de Chile, Valdivia, Chile

A. Paliathanasis

Institute of Systems Science, Durban University of Technology, Durban 4000, Republic of South Africa

E-mail: anpaliat@phys.uoa.gr 
has a direct sensory correspondence; therefore, 'couples' much easier and directly with the description of the physical phenomena.

In GR, the space used (spacetime) and the geometry employed (Riemannian geometry) do not allow for a direct comparison of the mathematical description with the direct sensorial reality. Most frequently, this results in a confusion as to where geometry starts and where it stops, how much Physics is used and done, what is the physical interpretation of the geometrically derived results, etc. On top of that it does not seem to exist at a reasonable level a concise exposition of the structure of GR as a theory of Physics and a clear description of the various hypotheses made both at the geometric and at the physical level when a gravitational model is presented. For example, it is widely misunderstood that Einstein's gravitational field equations [9] are equations in the usual sense, that is, they contain unknown quantities of some sort which are specified as soon as one "solves" them. However, on the contrary, these equations contain only unknowns and there is no way or point to solve them. In fact, they are generators of standard field equations which result after certain assumptions are made which specify a "model" gravitational universe and whose solutions reveal the properties of the specific model universe. This is the reason that there exist so many (and still are produced) "solutions" of the GR field equations in the literature [14,26].

The purpose of this article is to develop in a systematic way the structure of a generic model for GR so that one has a clear understanding of the impact of the geometric and the physical assumptions made in the construction of a certain gravitational model within the framework of GR.

The generic model of GR consists of two parts:

(a) The first part concerns all possible parameters which are used to build a GR model. These parameters are classified into two large sets: (i) geometric restrictions/assumptions and (ii) physical restrictions/requirements

(b) The second part consists of all constrains the parameters of the first part must satisfy to lead to a mathematically consistent relativistic model. These constrains are also classified into two large sets:

(i) constraints resulting from geometric identities and geometric relations in general and

(ii) constraints due to physical simplifications which have the general name equations of state.

The constrains in the two sets are not independent, in the sense that parameters from one set constraint the parameters in the second set and vice versa. This is due to the GR field equations which relate the geometry with Physics. It is apparent that when one makes assumptions on the Physics of a model, these assumptions have to be compatible with the geometric requirements of the model. If this is not the case, then there is the possibility that the proposed gravitational model is inconsistent, hence an invalid model.

The Physics today is still in its Aristotelian form, that is, a statement/prediction/result is either "true" (i.e., justified by the world "out there") or not. This is the logic of zero and one. As it is well known today the Aristotelian point of view has been replaced in many areas (especially the technological) by the Fuzzy Physics approach which is beyond the logic of true or false. This type of Physics has yet to come. However, because GR is definitely a theory of the Aristotelian type, we are not to worry about that type of future developments and we shall follow the classical path.

\section{The generic model of GR}

Although the theories of Physics follow the general pattern of the theories of other sciences, they have the unique characteristic that they use mathematical entities to model actual physical quantities of the real world. The association of these two very different type of entities imposes certain requirements which a theory of Physics must satisfy to be meaningful. In general terms, every theory of Physics must have the following ingredients.

\section{The background mathematical space}

Physics does not build its models in the 'real' space, i.e., the space we live in. This is the case only in Newtonian Physics where we have a direct sense of the evolution of physical systems in space due to the direct sensory conception we have for the Newtonian world. In relativistic models of Physics we have an 'indirect sensory' conception of the world by means of our measuring instruments. The first relativistic theory which was proposed was of course special relativity where people could not have a direct sensory feeling; therefore, 
they were unable to understand and anxious to disprove it by means of the many paradoxes proposed. In short, it was violating their 'common sense', the latter being possible to be put forward as the rule "I see it, I believe it"!

The trouble is the use of the word 'space' to describe both a mathematical entity (software) and a physical entity (hardware). The physical entity is what it is 'out there'. The mathematical entity is a set with certain mathematical structures. Our intention here is not to enter into details about these delicate matters, which in any case are not first priority questions to a general physicist.

The mathematical space used by the theories of Physics is a point set which has the structure of a manifold. The main characteristic of the manifold structure is that it is locally diffeomorphic to an open set in a flat space of some dimension (called the dimension of the manifold); therefore, it can be covered by a set of coordinate patches from the flat space in such a way that whenever two coordinate patches coincide, there is a differentiable map which relates them either way. In general relativity, the set of points is the events which are assumed to correspond to the various events in the real world. The coordinate patches are called coordinate systems and are diffeomorphic to open neighborhoods of $R^{4}$. The differentiable maps which relate two coordinate systems are called coordinate transformations. This manifold is called spacetime. ${ }^{1}$

The coordinate transformations form a group under the action of composition of maps. This group is an infinite dimensional Lie group called the Manifold Mapping Group (MMG) [1]. The mathematical quantities which in any coordinate system are described by means of a set of components so that under coordinate transformations they transform in a definite way we call geometric objects. We say that the geometric objects form a representation of the MMG. The nature of a geometric object is characterized by the way its components transform under coordinate transformations in the manifold. The geometric objects which transform in a linear and homogeneous manner we call tensors. In the following we shall restrict our considerations to tensors.

This is as far as one can go with the assumption of manifold structure of the background mathematical space. However, this mathematical structure is not enough to study the physical phenomena because it lacks the concept of "measure". It is safe to say that in all theories of Physics the concept of "measure" is introduced by the requirement of the existence of a specific geometric object on the manifold which is the metric. Let us see how this is done.

It is possible (but not necessary!) that besides the MMG group, a theory of Physics introduces an additional characteristic subgroup of the MMG. This is achieved by considering an additional inherent structure by means of an absolute ${ }^{2}$ metric tensor defined all over the space. This subgroup defines special classes of tensors by the requirement that they transform covariantly under the coordinate transformations derived by the special subgroup. For example, in special relativity one assumes the Lorentz metric whose canonical form ${ }^{3}$ is preserved under the coordinate transformations which we know as Lorentz transformations and are elements of the Lorentz group. The geometric objects which transform covariantly under the Lorentz transformations we call Lorentz tensors. Similarly, in Newtonian Physics one assumes the Euclidian metric whose canonical form is the tensor $\delta_{\mu \nu}$. The coordinate transformations which preserve $\delta_{\mu \nu}$ we call Galilean transformations and they form the Galilean group which defines the Newtonian tensors. In GR we do not assume an absolute metric $^{4}$; hence, there does not exist a special subgroup and the geometric objects of the theory are general tensors.

\subsection{The role of geometry}

The introduction of a metric, which is a tensor or order $(0,2)$, in a general manifold defines an additional structure which we call geometry. Using geometry one is able to define a correspondence of the geometric objects with physical entities and define the covariant derivative which is necessary in the formulation of the Laws of Physics. Let us see how this is done in the classical theories of Physics.

\footnotetext{
${ }^{1}$ In addition to the local structure spacetime, there exists a global structure described by its topology. However, these topological properties are not of our interest.

${ }^{2}$ By absolute we mean that there exist coordinate systems called inertial coordinate systems which are defined all over the space and in which the metric has its canonical form, that is, it is represented by a diagonal square matric whose elements are \pm 1 . The linear coordinate transformations preserve the canonical form of the metric forma group which is the characteristic group of the theory.

${ }^{3}$ Canonical form of a metric in a coordinate system is defined by the requirement that in that coordinate system the metric is represented with a diagonal matrix whose entries are \pm 1 .

4 Assumes only that at each point there is a coordinate system in which the metric takes its canonical form which is the canonical form of the Lorentz metric, i.e., $\operatorname{diag}(-1,1,1,1)$.
} 
In Newtonian Physics the physical requirement is that there exist Euclidian solids, that is, objects whose points are such that their Euclidian distance does not change as they change their state. This postulates the absolute character of the Euclidian metric. The geometry defined in the 3D manifold $R^{3}$ by the Euclidian metric is the so-called Euclidian geometry which makes the space to be the Euclidian space $E^{3}$. In practical terms, this means that under linear transformations which are isometries of the Euclidian metric $E^{3}$ the laws of Physics stay the same. These transformations form a group which is called the Galilean group. The tensors of the space which are transformed covariantly with respect to the Galilean group are called Newtonian tensors. All Newtonian Physics develops on the space $E^{3}$ and all Newtonian physical quantities are described by Newtonian tensors. Furthermore, all Newtonian Laws relate Newtonian tensors only.

In special relativity, the physical requirement is the invariance of the speed of light. Extending this to more general than the light phenomena leads to the postulation of the Lorentz metric and the introduction of the Minkowski space $M^{4}$ where the Physics of special relativity is done. The isometry group of the Lorentz metric is the Lorentz group (or more correctly the Poincare group) whose linear transformations are the Lorentz transformations. These transformations define the special relativistic tensors. It is important to note that the Physics which is based on $M^{4}$ will be different from the Newtonian Physics which is based on $E^{3}$. This means that the relativistic phenomena are not necessarily Newtonian and certainly the Newtonian are not relativistic. For instance, there are no Euclidian solids in Special Relativity. Therefore, there is no point to say that one theory is wrong and the other correct. Simply each theory (provided it has a "touch" with reality) applies to its own physical phenomena. Of course, people would like to have the "theory of everything" but this is another characteristic aspect of the human utopia.

Concerning GR the situation is different. The main interest of GR is to develop a theory for the gravitational field while at the same time to reduce to special relativity when the gravitational field is switched off. Therefore, in this theory one keeps the notion of metric and associates it with the gravitational field. The difference with the previous two theories is that there is no globally defined metric; therefore, there does not exist a characteristic subgroup of the MMG group for this theory. This implies that the tensors which are used in GR are general tensors which transform linearly and homogeneously with respect to the transformations of the MMG group. Furthermore, because at each point we want the theory to reduce to Special Relativity, it is postulated that the signature of the metric will be that of the Lorentz metric, that is, -2 .

The result of considering a general metric is that in the manifold of GR one defines a Riemannian geometry (i.e., a geometry with curvature) which is characterized by the introduction of the notion of the Riemannian covariant derivative. The latter is defined by the requirements that it is symmetric (the torsion tensor vanishes) and also metrical (the covariant derivative of the metric vanishes, or as we say, it has zero metricity). The manifold structure endowed with the Riemannian geometry we call spacetime. Spacetime is the background space of general relativity where all gravitational phenomena shall be considered.

The introduction of Riemannian geometry has many new effects which are hidden in the spaces $E^{3}$ and $M^{4}$ whose geometry is also Riemannian but trivial. These effects are the following.

- Using the metric and its derivatives one introduces many new geometric objects which vanish in $E^{3}$ and $M^{4}$. These are the connection coefficients, the Ricci tensor $R_{a b}$, the curvature tensor $R_{a b c d}$, etc. With a collective name we shall call them metric tensors.

- There are geometric identities which must be satisfied by the metric tensors. These main identities are the following:

(a) The Ricci identity: for a tensor of type $(r, s)$ this identity is

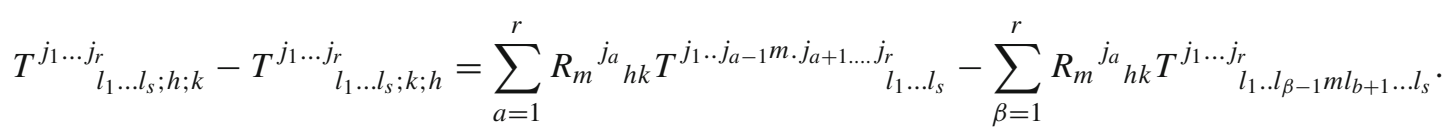

In particular, for a vector field $X^{a}$ Ricci identity reads:

$$
X_{a ; b c}-X_{a ; c b}=R_{d a b c} X^{d} .
$$

(b) The Bianchi identities:

$$
R_{a b[c d ; e]}=0
$$




$$
C_{. ; d}^{a b c d}=R^{c[a ; b]}-\frac{1}{6} g^{c[a} R^{; b]},
$$

where $C_{a b c d}$ is the Weyl tensor ${ }^{5}$ In a four-dimensional Riemannian space, these two identities are equivalent ${ }^{6}$; therefore, in spacetime they reduce to one identity. These identities imply the important contracted Bianchi identity:

$$
R_{\ldots ; b}^{a b}-\frac{1}{2} R^{; a}=0 \Leftrightarrow G_{\ldots ; b}^{a b}=0,
$$

where

$$
G_{a b}=R_{a b}-\frac{1}{2} R g_{a b}
$$

is the Einstein tensor.

- A third result of the geometrical structure is the possibility it provides to introduce new geometric requirements which will act as constraints additional to the inherent ones assumed by the Riemannian structure of spacetime. The major type of such geometric constraints are the "symmetries" or collineations of the metric objects. A symmetry or collineation is a relation of the form

$$
L_{X} A=B
$$

where $X$ is a vector field and $A, B$ are metric geometric objects with the same number of indices and the same symmetry properties of the indices. The following are examples of collineations [13]:

$$
\begin{array}{ll}
\mathcal{L}_{\xi} g_{a b}=0 & \text { Killing Vector } \\
\mathcal{L}_{\xi} g_{a b}=2 c g_{a b}, c=\text { const. } & \text { Homothetic Killing Vector } \\
\mathcal{L}_{\xi} g_{a b}=2 \psi g_{a b}, \psi \neq \text { const. } & \text { Conformal Killing Vector } \\
\mathcal{L}_{\xi} \Gamma_{j k}^{i}=0 & \text { Affine collineation Vector } \\
\mathcal{L}_{\xi} R_{a b}=0 & \text { Ricci collineation } \\
\mathcal{L}_{\xi} R_{j k l}^{i}=0 & \text { Curvature collineation } \\
g^{a b} \mathcal{L}_{\xi} R_{a b}=0 & \text { Contracted Ricci collineation } \\
\mathcal{L}_{\xi} W_{j k l}^{i}=0 & \text { Contracted Ricci collineation. }
\end{array}
$$

- Other geometrical constraints. These are mathematical requirements introduced 'by hand' after the previous two levels of simplifying assumptions have been exhausted. The purpose of their introduction is to simplify further the equations obtained by the previous simplifying assumptions. Their form depends on the form of the equations they have to simplify. For example, in the case of a Conformal Killing Vectors (CKV) such a requirement is $\psi_{; a b}=0$ because this condition removes $\psi_{; a b}$ from the field equations. A CKV which satisfies this condition is called a special CKV.

It is to be noted that up to now our discussion is limited to the background space only; therefore, the results apply to all physical fields introduced or, to be more specific, to both the kinematics and the dynamics of the whatever gravitational theory will be developed on this spacetime.

\subsection{Some useful material about collineations}

Each collineation has a different constraint 'power'. A collineation is called proper if it cannot be reduced to a 'simpler' one. For example, the CKVs contain the homothetic Killing vectors (HKV) when the conformal function $\psi=$ constant. A proper CKV is one for which $\psi$ is not constant.

The vectors which satisfy $\mathcal{L}_{\xi} g_{a b}=0$ are called Killing vectors (KVs) and the equation $\mathcal{L}_{\xi} g_{a b}=0$ Killing equation. In an $\mathrm{n}$-dimensional space with a non-degenerate metric, there exist at most $\frac{n(n+1)}{2} \mathrm{KV}$ s. If this is the case then the space is called a space of constant curvature. The KVs serve all standard geometric symmetry concepts, e.g. spherical symmetry, cylindrical symmetry, etc.

\footnotetext{
5 To be defined in the following.

6 This equivalence holds for $n=4$ only. See Kundt and Trumper Abh. Akad. Wiss. and Lit. Mains. Mat. Nat. Kl. No. 12 (1962).
} 
The KVs form a finite-dimensional Lie algebra. This algebra can be used to classify the metrics in various classes in the sense that one is able to write the metric in a form that takes into account the symmetry and it is written in terms of a small number of parameters.

A well-known example is the Friedmann-Robertson-Walker (FRW) spacetime which is used in the standard cosmology. The metric of this spacetime has the form:

$$
\mathrm{d} s_{R W}^{2}=-\mathrm{d} t^{2}+R(t)^{2}\left(\mathrm{~d} x^{2}+\mathrm{d} y^{2}+\mathrm{d} z^{2}\right),
$$

where $(t, x, y, z)$ are Cartesian coordinates and $R(t)$ is the only unknown function of (the coordinate) time $t$ and can be defined by means of the following symmetry assumptions [28]:

1. Spacetime admits a timelike gradient Conformal Killing Vector (CKV) such that there exists another reduced metric for which the $\mathrm{CKV}$ is a $\mathrm{KV}$.

2. The 3-D hypersurfaces orthogonal to the CKV are spaces of constant curvature, consequently the FRW spacetime admits six KVs.

The collineations other than Killing vectors fix the metric to a lesser degree; however, they do act as constraints and can be used in any case as such.

\subsection{The generic collineation}

One can prove that the Lie derivative of every metric geometric object in Riemannian geometry can be expressed in terms of the quantity $\mathcal{L}_{\xi} g_{a b}$ and its derivatives. For instance, the following relations are true:

$$
\begin{aligned}
L_{\xi} \Gamma_{a b}^{c} & =\frac{1}{2} g^{c d}\left[\left(L_{\xi} g_{a d}\right)_{; b}+\left(L_{\xi} g_{b d}\right)_{; a}-\left(L_{\xi} g_{a b}\right) ; d\right] \\
L_{\xi} R_{b c d}^{a} & =\left(L_{\xi} \Gamma_{b d}^{a}\right)_{; c}-\left(L_{\xi} \Gamma_{b c}^{a}\right)_{; d} \\
L_{\xi} R_{a b} & =\left(L_{X} \Gamma_{a b}^{c}\right)_{; c}-\left(L_{X} \Gamma_{c a}^{c}\right)_{; b} .
\end{aligned}
$$

This observation leads us to introduce the concept of generic collineation by the relation/identity:

$$
L_{\xi} g_{a b}=2 \psi g_{a b}+2 H_{a b},
$$

where $H_{a b}$ is a symmetric traceless tensor. Then it is possible to express every collineation in terms of the symmetry variables $\psi, H_{a b}$ and their derivatives. This approach greatly unifies and simplifies the study of the effects of each collineation and reveals its relative significance.

As an example we refer the following general result:

$$
L_{\xi} R_{a b}=-(n-2) \psi_{; a b}-g_{a b} \square \psi+2 H_{.(a ; b) d}^{d}-\square H_{a b} .
$$

In terms of trace and traceless parts $L_{\xi} R_{a b}$ is written as follows:

$$
L_{\xi} R_{a b}=-(n-2) A_{a b}+2 K_{a b}-\square H_{a b}+\frac{1}{n} g_{a b}\left[-2(n-1) \square \psi+2 H_{: ; a b}^{a b}\right],
$$

where the traceless tensors:

$$
A_{a b}=\psi_{; a b}-\frac{1}{n} g_{a b} \square \psi \text { and } K_{a b}=H_{.(a ; b) d}^{d}-\frac{1}{n} g_{a b} H_{. ; c d}^{c d} .
$$

In spacetime $(n=4)$, the above formulae read:

$$
\begin{aligned}
L_{\xi} R_{a b} & =-2 \psi_{; a b}-g_{a b} \square \psi+2 H_{.(a ; b) d}^{d}-\square H_{a b}, \\
L_{\xi} R_{a b} & =-2 A_{a b}+2 K_{a b}-\square H_{a b}+\frac{1}{4} g_{a b}\left(-6 \square \psi+2 H_{. ; a b}^{a b}\right), \\
A_{a b} & =\psi_{; a b}-\frac{1}{4} g_{a b} \square \psi \text { and } K_{a b}=H_{.(a ; b) d}^{d}-\frac{1}{4} g_{a b} H_{. ; c d}^{c d} .
\end{aligned}
$$

This is as far as one can go with the spacetime structure (i.e., the introduction of the metric tensor). It is possible to continue making geometry in spacetime but not Physics. To do the latter, one has to introduce more tensor fields which will describe/correspond to the physical quantities. 


\section{The observers: kinematics}

The main purpose of all physical theories is to "explain" the real world (whatever "real" means) to us, the observers who observe the physical quantities and describe their evolution in the cosmos. Therefore, all theories of Physics must have two types of entities: observers and observed phenomena. In non-quantum Physics, these two entities are separate in the sense that they do not interact. In quantum Physics this is not the case and one has the so-called Uncertainty Principle which, however, will not concern us here. Both types of entities must be described mathematically in the space of the theory by a certain type of geometric object.

In GR, the observers are represented in the same way as in Special Relativity, that is, by a timelike world line whose tangent unit four vector $u^{a}$ is the four velocity of the observers. In GR, there is no way to define a special class of inertial observers because it is not assumed to exist a characteristic group of transformations defined all over the spacetime manifold. Instead of them, one defines the closest type of observers which are the free-falling observers whose spacetime trajectory is a geodesic of the metric tensor considered by the specific model. All other classes of observers in the same spacetime are characterized as accelerating observers. We note that each gravitational model in GR has its own free-falling observers depending on the metric it adopts. Also when the gravitational field is switched off the geodesics are straight lines in the space $M^{4}$; therefore, the observers coincide with those of Special Relativity.

The world lines of a class of observers in GR constitute the 'fluid' of observers in spacetime and this fluid is used to express the kinematic properties of these observers in terms of this fluid flow quantities.

As we have already mentioned the assumed structure of Riemannian geometry of spacetime introduces identities among the tensor fields which act as "internal" constraints universal to all models one may consider. In addition to these "internal" identities, each model theory introduces new geometric relations which act as "external" constraints to the theory. The two types of constraints must be satisfied by the four velocity vector of the observers of the model theory. This fact defines the first level of the physical theory which is called kinematics and it involves only the considered observers and the geometric constraints (internal and external) imposed by the model. On a given kinematics, one is possible to build various models by defining a different dynamics of the theory.

The introduction of a non-null vector field in spacetime allows one to decompose a tensor equation and an individual tensor parallel and normal to the vector field. The vector field can be either timelike or spacelike. This decomposition is called $1+3$ decomposition for obvious reasons. It is of primary importance to Physics because it is covariant, in the sense that each irreducible part transforms under coordinate transformations independently of the other. Therefore, one can break the study of the Physics (kinematics and dynamics) of a tensor field or a field equation by studying the simpler Physics of its irreducible parts.

Below we develop the basic mathematics of the $1+3$ decomposition with respect to a non-null vector field $P^{a}$. The results of this analysis can be extended easily to the $1+(n-1)$ decomposition in an $n$-dimensional spacetime by making the necessary adjustments.

\section{$4.11+3$ decomposition wrt a non-null vector field}

Consider a non-null $\left(P^{i} P_{i} \neq 0\right)$ vector field $P^{i}$ with signature $\varepsilon(P)=P^{i} P_{i} /\left|P^{i} P_{i}\right|= \pm 1$, where the +1 applies to a spacelike four-vector and the -1 to a timelike four-vector in a metric space with metric $g_{i j}$. The projection tensor $h_{i j}(P)$ associated with $P^{i}$ is defined by the equation:

$$
h_{i j}(P)=g_{i j}-\frac{\varepsilon(P)}{P^{2}} P_{i} P_{j},
$$

where $P^{2}=\left|P^{i} P_{i}\right|=\varepsilon(P) P^{i} P_{i}>0$. In a four-dimensional manifold, it is easy to prove the properties:

$$
h_{i j}(P) P^{j}=0, h^{i j}(P) h_{j k}(P)=h_{k}^{i}(P), h_{i}^{i}(P)=3 .
$$

The tensor $h_{i j}(P)$ projects normally to $P^{a}$ and gives us the possibility to decompose any tensor field in irreducible parts in a direction parallel to $P^{a}$ and another normal to $P^{a}$, as given by the following propositions.

Proposition 4.1 A general vector field $R^{i}$ can be $1+3$ decomposed wrt $P^{i}$ as follows:

$$
R^{i}=\alpha P^{i}+\beta^{j} h_{j}^{i}
$$


where

$$
\begin{aligned}
\alpha & =\frac{\varepsilon(P)}{P} R^{i} P_{i} \\
\beta^{i} & =R^{i} .
\end{aligned}
$$

Proof We have $R^{i}=R^{j} g_{j}^{i}$. Using (19) this gives:

$$
\begin{aligned}
R^{i} & =R^{j} g_{j}^{i} \\
& =R^{j}\left(\frac{\varepsilon(P)}{P^{2}} P_{j} P^{i}+h_{j}^{i}\right) \\
& =\frac{\varepsilon(P)}{P^{2}}\left(R^{j} P_{j}\right) P^{i}+h_{j}^{i} R^{j} .
\end{aligned}
$$

Proposition 4.2 A general second-order tensor $Y_{i j}$ is $1+3$ decomposed wrt the vector $P^{i}$ by means of the identity:

$$
Y_{i j}=\alpha P_{i} P_{j}+\varepsilon(P) \beta_{k} h_{j}^{k} P_{i}+\varepsilon(P) \gamma_{k} h_{i}^{k} P_{j}+\epsilon_{i j},
$$

where

$$
\alpha=\frac{1}{P^{4}} Y_{i j} P^{i} P^{j}, \beta_{i}=\frac{1}{P^{2}} Y_{i j} P^{j}, \gamma_{i}=\frac{1}{P^{2}} Y_{j i} P^{j}, \epsilon_{i j}=Y_{k r} h_{i}^{k} h_{j}^{r}
$$

Proof We write $Y_{i j}=Y_{k r} g_{i}^{k} g_{j}^{r}$ and using (19) we have

$$
\begin{aligned}
Y_{i j} & =Y_{k r} g_{i}^{k} g_{j}^{r} \\
& =Y_{k r}\left(\left(\frac{1}{P^{2}} \varepsilon(P) P^{k} P_{i}+h_{i}^{k}\right)\left(\frac{1}{P^{2}} \varepsilon(P) P^{r} P_{j}+h_{j}^{r}\right)\right. \\
& =\left(\frac{1}{P^{4}} Y_{k r} P^{k} P^{r}\right) P_{i} P_{j}+\frac{1}{P^{2}} \varepsilon(P)\left(Y_{k r} P^{k}\right) P_{i} h_{j}^{r}+\frac{1}{P^{2}} \varepsilon(P)\left(Y_{k r} P^{r}\right) h_{i}^{k} P_{j}+Y_{k r} h_{i}^{k} h_{j}^{r} \\
& =\alpha P_{i} P_{j}+\varepsilon(P) \beta_{k} h_{j}^{k} P_{i}+\varepsilon(P) \gamma_{k} h_{i}^{k} P_{j}+\epsilon_{i j} .
\end{aligned}
$$

We note that a symmetric tensor of rank 2 is specified (and equivalently specifies) in terms of five different quantities: one scalar, two vector and one projected second rank tensor.

\section{$4.21+3$ decomposition wrt a timelike unit vector field}

In the following, we consider $P^{i}$ to be a normalized timelike vector field $(\varepsilon(P)=-1)$ (e.g. a four-velocity) and we denote it $u^{i}$. Then Eqs. (19), (20) and (23) give the $1+3$ decomposition wrt $u^{i}\left(u^{i} u_{i}=-1\right)$ :

$$
\begin{aligned}
h_{i j}(u) & =g_{i j}+u_{i} u_{j} \\
R^{i} & =-\left(R^{j} u_{j}\right) u^{i}+R^{j} h_{j}^{i}(u) \\
Y_{i j} & =\left(Y_{k r} u^{k} u^{r}\right) u_{i} u_{j}-\left(Y_{k r} u^{k}\right) u_{i} h_{j}^{r}-\left(Y_{k r} u^{r}\right) h_{i}^{k} u_{j}+Y_{k r} h_{i}^{k} h_{j}^{r} .
\end{aligned}
$$

For a symmetric tensor of type $(0,2)$ decomposition $(26)$ is written as follows:

$$
Y_{a b}=\left(Y^{r s} u_{r} u_{s}\right) u_{a} u_{b}-Y^{r s} u_{r} h_{s b} u_{a}-Y^{r s} u_{s} h_{r a} u_{b}+\frac{1}{3}\left(Y^{r s} h_{r s}\right) h_{a b}+\left(h_{a}^{r} h_{b}^{s}-\frac{1}{3} h_{a b} h^{r s}\right) Y_{r s},
$$

that is, we brake further the symmetric part in a trace and a traceless part.

We consider now various applications of the $1+3$ decomposition wrt the vector field $u^{a}$. 


\subsubsection{The kinematic variables of the four-velocity}

We perform the $1+3$ decomposition of the tensor $u_{i ; j}$. By definition, from (24) we have the following identity/decomposition:

$$
u_{i ; j}=\left(u_{k ; r} u^{k} u^{r}\right) u_{i} u_{j}-\left(u_{k ; r} u^{k}\right) u_{i} h_{j}^{r}-\left(u_{k ; r} u^{r}\right) h_{i}^{k} u_{j}+u_{k ; r} h_{i}^{k} h_{j}^{r} .
$$

Moreover, $u_{i ; j} u^{i}=\frac{1}{2}\left(u_{i} u^{i}\right)_{; j}=0$ and $u_{i ; j} u^{i}=\dot{u}_{j}=h_{j}^{r} \dot{u}_{r}$. Therefore, identity (28) is simplified as follows:

$$
u_{i ; j}=-\dot{u}_{i} u_{j}+u_{k ; r} h_{i}^{k} h_{j}^{r}
$$

We continue by decomposing the spacelike part $u_{k ; r} h_{i}^{k} h_{j}^{r}$ in an antisymmetric and a symmetric part as follows:

$$
\begin{aligned}
u_{k ; r} h_{i}^{k} h_{j}^{r} & =\omega_{i j}+\theta_{i j} \\
\omega_{i j} & =u_{k ; r} h_{[i}^{k} h_{j]}^{r} \\
\theta i j & =u_{k ; r} h_{(i}^{k} h_{j)}^{r} .
\end{aligned}
$$

The symmetric part can be decomposed covariantly further to a trace and a traceless part, that is, we write:

$$
\theta_{i j}=\sigma_{i j}+\frac{1}{3} \theta h_{i j}
$$

where

$$
\begin{aligned}
\theta & =\theta_{i}^{i}=h^{i j} u_{i ; j} \\
\sigma_{i j} & =\theta_{i j}-\frac{1}{3} \theta h_{i j}=\left[h_{(i}^{r} h_{r)}^{k}-\frac{1}{3} h^{r k} h_{i j}\right] u_{r ; k} .
\end{aligned}
$$

The term $\omega_{i j}$ is called the vorticity tensor of $u^{i}, \sigma_{i j}$ the shear tensor of $u^{i}, \theta$ the expansion of $u^{i}$ and $\dot{u}^{i}$ the four-acceleration of the timelike vector field $u^{i}$. These are the kinematic variables of $u^{i}$, considering $u^{i}$ to be the four-velocity of a relativistic fluid.

We infer by their definition that the kinematic variables satisfy the properties:

$$
\begin{aligned}
\omega_{i j} & =-\omega_{j i}, \omega_{i j} u^{j}=0 \\
\sigma_{i j} & =\sigma_{j i}, \sigma_{i}^{i}=\sigma_{i j} u^{j}=0 \\
\omega_{i j} & =h_{i}^{k} h_{j}^{r} \omega_{k r} \\
\sigma_{i j} & =h_{i}^{k} h_{j}^{r} \sigma_{k r} .
\end{aligned}
$$

The geometric meaning of each kinematic term is obtained from the study of the integral curves of $u^{a}$. We shall not comment further on that at this point.

We interpret the physical meaning each quantity in terms of relative motion. In this respect, we say that $\theta$ is expansion (isotropic strain), $\sigma_{a b}$ is shear (anisotropic strain), $\omega_{a b}$ relative rotation and $\dot{u}_{a}$ denotes the fouracceleration. These quantities (i.e., $\sigma_{a b}, \omega_{a b}, \dot{u}_{a}, \theta$ ) are the fundamental physical quantities of relativistic (and Newtonian with the necessary adjustments) kinematics.

Two scalars of special interests are the following:

$$
\begin{aligned}
\sigma^{2} & =\frac{1}{2} \sigma_{a b} \sigma^{a b}, \\
\omega^{2} & =\frac{1}{2} \omega_{a b} \omega^{a b} .
\end{aligned}
$$

To demonstrate the geometric importance of the kinematic quantities, we refer the following proposition.

Proposition 4.3 Necessary and sufficient conditions for the vector field $u^{i}$ to be a Killing vector is:

$$
\sigma_{i j}=0, \theta=0, \dot{u}^{i}=0 .
$$


Proof We have

$$
L_{u} g_{i j}=2 u_{(i ; j)}=2 \sigma_{i j}+\frac{2}{3} \theta h_{i j}-2 \dot{u}_{(i} u_{j)} .
$$

For $u^{i}$ to be a Killing vector it should satisfy the condition $L_{u} g_{i j}=0$. Combining these two relations, we find:

$$
\sigma_{i j}=0, \theta=0, \dot{u}^{i}=0
$$

\section{The propagation and the constraint equations}

As we remarked above, the vector field of the four-velocity decomposes both the geometric objects of the theory as well as the covariant equations among them. The Riemannian structure of spacetime has two major geometric identities: the Ricci identity and the Bianchi identities. The $1+3$ decomposition of these identities produces the necessary equations which must be satisfied by all the kinematic quantities involved.

The $1+3$ decomposition of the Ricci identity leads to two sets of equations each set containing nine equations. The first set contains the derivatives of the kinematic quantities along $u^{a}$ which we call the propagation equations. The second set of equations is called the constraint equations [11]. The two sets of equations are as follows:

Propagation equations ${ }^{7}$ :

$$
\begin{aligned}
& h_{a}^{b} \dot{\omega}^{a}=\left(\sigma_{d}^{a}-\frac{2}{3} \theta h_{d}^{a}\right) \omega^{d}+\frac{1}{2} \eta^{a b c d} u_{b} \dot{u}_{c ; d} \quad \text { (three equations) } \\
& \dot{\theta}+\frac{1}{3} \theta^{2}+2\left(\sigma^{2}-\omega^{2}\right)=-R_{a b} u^{a} u^{b}+\dot{u}_{; a}^{a} \text { (one equation) } \\
& -E_{s t}+\frac{1}{2}\left(h_{s}^{a} h_{t}^{b}-\frac{1}{3} h_{s t} h^{a b}\right) R_{a b}=h_{s}^{a} h_{t}^{b}\left[\dot{\sigma}_{a b}-\dot{u}_{(a ; b)}\right]+\sigma_{s c} \sigma_{t}^{c}+\text { (five equations) } \\
& +\frac{2}{3} \sigma_{s t} \theta+\omega_{s} \omega_{t}-\dot{u}_{s} \dot{u}_{t}-\frac{1}{3}\left(2 \sigma^{2}+\omega^{2}-\dot{u}_{; b}^{b}\right) h_{s t} .
\end{aligned}
$$

Constraint equations:

$$
\begin{aligned}
& h_{b}^{a} \omega_{; a}^{b}=\dot{u}^{a} \omega_{a} \text { (one equation), } \\
& h_{s}^{c}\left[\frac{2}{3} \theta,{ }_{c}-h^{a b} \sigma_{c a ; b}-\eta_{c a m n} u^{a}\left(\omega^{m ; n}+2 \omega^{m} \dot{u}^{n}\right)\right]=-h_{s}^{c} R_{c d} u^{d} \text { (three equations), } \\
& -h_{(s}^{a} h_{l)}^{b}\left[\sigma_{b}^{c ; d}+\omega_{b}^{c ; d}\right] \eta_{a r c d} u^{r}+2 \dot{u}_{(s} \omega_{l)}=H_{s l} \text { (five equations). }
\end{aligned}
$$

\section{The $1+3$ decomposition of the Bianchi identity}

The $1+3$ decomposition of the Bianchi identity (4) involves the Weyl tensor.

It is well known that the Riemann tensor can be decomposed into the following irreducible parts as follows:

$$
R_{a b c d}=C_{a b c d}+\frac{1}{2}\left(g_{a c} R_{b d}+g_{b d} R_{a c}-g_{a d} R_{b c}-g_{b c} R_{a d}\right)-\frac{1}{6} R g_{a b c d},
$$

\footnotetext{
${ }^{7}$ Note that the propagation equation of $\omega_{a b}$ (37) contains only kinematic terms and it is independent of $R_{a b}$, hence of the dynamical variables to be introduced later on.
} 
where

$$
g_{a b c d}=g_{a c} g_{b d}-g_{a d} g_{b c}
$$

and $C_{a b c d}$ is the Weyl tensor. The Weyl tensor has the same symmetries of indices as the curvature tensor but all its traces vanish. Equation (43) is a mathematical identity. The Weyl tensor is decomposed further in terms of the electric part $E_{a b}$ and the magnetic part $H_{a b}$ wrt the vector field $u^{a}$ as follows:

$$
C_{a b c d}=\left(g_{a b r s} g_{c d m t}-\eta_{a b r s} \eta_{c d m t}\right) u^{r} u^{m} E^{s t}-\left(\eta_{a b r s} g_{c d m t}+g_{a b r s} \eta_{c d m t}\right) u^{r} u^{m} H^{s t},
$$

where

$$
E_{a c}=C_{a b c d} u^{b} u^{d}, \quad H_{a c}=\frac{1}{2} \eta_{a m}^{k l} C_{k l b t} u^{m} u^{t} .
$$

The tensors $E_{a c}, H_{a c}$ are symmetric, traceless and satisfy the property:

$$
E_{a c} u^{c}=H_{a c} u^{c}=0 .
$$

If in (4) we substitute the Weyl tensor $C^{a b c d}$ in terms of the electric and the magnetic part $E^{a b}, H^{a b}$ we obtain four identities which have a form similar to Maxwell equations for the electric and the magnetic fields:

$\nabla E$ :

$$
\begin{aligned}
h_{a}^{t} h_{s}^{d} E_{; d}^{a s}-\eta^{t b p q} u_{b} \sigma_{p}{ }^{d} H_{q d}+3 H_{s}^{t} \omega^{s}= & \frac{1}{3} h^{t b} \mu_{; b}-\frac{1}{2} h_{c}^{t} \pi_{; b}^{c b}-\frac{3}{2} \omega_{. b}^{t} q^{b} \\
& +\frac{1}{2} \sigma_{. b}^{t} q^{b}+\frac{1}{2} \pi_{. b}^{t} \dot{u}^{b}-\frac{1}{3} \theta q^{t},
\end{aligned}
$$

$\nabla H:$

$$
\begin{aligned}
h_{a}^{t} h_{s}^{d} H_{; d}^{a s}+\eta^{t b p q} u_{b} \sigma_{. p}^{d} E_{q d}-3 E_{. s}^{t} \omega^{s}= & (\mu+p) \omega^{t}+\frac{1}{2} \eta^{t b s f} u_{b} q_{s ; f} \\
& +\frac{1}{2} \eta^{t b s f} u_{b} \pi_{s c}\left(\omega_{f}^{c}+\sigma_{f}^{. c}\right),
\end{aligned}
$$

$\dot{E}_{a b}$ :

$$
\begin{aligned}
{\left[\begin{array}{c}
h_{a}^{m} h_{c}^{t} \dot{E}^{a c}+h_{a}^{(m} \eta^{t) r s d} u_{r} H_{. s ; d}^{a}-2 H_{q}^{(t} \eta^{m) b p q} u_{b} \dot{u}_{p} \\
+h^{m t} \sigma^{a b} E_{a b}+\theta E^{m t}-3 E_{s}^{(m} \sigma^{t) s}-E_{s}^{(m} \omega^{t) s}
\end{array}\right]=} & -\frac{1}{2}(\mu+p) \sigma^{t m}-\dot{u}^{(t} q^{m)}-\frac{1}{2} h_{a}^{t} h_{c}^{m} q^{(a ; c)} \\
& -\frac{1}{2} h_{a}^{t} h_{c}^{m} \dot{\pi}^{a c}-\frac{1}{2} \pi^{b(m}\left(\omega_{b}^{. t)}+\sigma_{b}^{. t)}\right)-\frac{1}{6} \pi^{t m} \theta \\
& +\frac{1}{6} h^{m t}\left(q_{. ; a}^{a}+\dot{u}_{a} q^{a}+\pi^{a b} \sigma_{a b}\right)
\end{aligned}
$$

$\dot{H}_{a b}:$

$$
\begin{aligned}
{\left[\begin{array}{c}
h^{m a} h^{t c} \dot{H}_{a c}-h_{a}^{(m} \eta^{t) r s d} E_{. s ; d}^{a}+2 E_{q}^{(t} \eta^{m) b p q} u_{b} \dot{u}_{p} \\
+h^{m t} \sigma^{a b} H_{a b}+\theta H^{m t}-2 H_{s}^{(m} \sigma^{t) s}-H_{s}^{(m} \omega^{t) s}
\end{array}\right]=} & \frac{1}{2} \sigma_{c}^{(t} \eta^{m) b c f} u_{b} q_{f}-\frac{1}{2} h_{c}^{(t} \eta^{m) b e f} u_{b} \pi_{. e ; f}^{c} \\
& +\frac{1}{2}\left(h^{m t} \omega_{c} q^{c}-3 \omega^{(m} q^{t)}\right) .
\end{aligned}
$$

The contracted Bianchi identity $G_{. . ; b}^{a b}=0$ is contained in the above identities. 


\section{Propagation of the kinematic quantities along a collineation vector}

The propagation equations provide the derivative of the kinematic quantities along the four-velocity $u^{a}$ of the observers due to the internal structure of spacetime. However, when a collineation is assumed by a given model then this is an external geometric constraint which must also be satisfied. This makes necessary the knowledge of the change of the kinematic quantities along the vector field generating the collineation. Because the collineations are defined in terms of the Lie derivative (Lie transport) we are interested in the quantities $L_{\xi}\left\{u^{a}, \omega_{a b}, \sigma_{a b}, \theta, \dot{u}^{a}\right\}$. We give the following general result.

Proposition 7.1 Let $X^{a}$ be a non-null vector field with index $\varepsilon(X)= \pm 1$, i.e., $X^{a} X_{a}=\varepsilon(X) X^{2}(X>0)$ and let $\xi^{a}$ be an arbitrary vector field with collineation parameters (see [29]) $\psi, H_{a b}$, that is,

$$
\xi_{(a ; b)}=\psi g_{a b}+H_{a b}=\frac{1}{2} L_{\xi} g_{a b}, \quad L_{\xi} g^{a b}=-2 \psi g^{a b}-2 H^{a b} .
$$

Then $L_{\xi} X^{a}$ is $1+3$ decomposed wrt the four-velocity $u^{a}$ as follows:

$$
L_{\xi} X^{a}=\left[(\ln X)_{, b} \xi^{b}-\psi-\frac{\varepsilon(X)}{X^{2}} H_{c b} X^{c} X^{b}\right] X^{a}+V^{a}(X)
$$

where $V^{a}(X)=h_{b}^{a}(X)\left(L_{\xi} X^{b}\right)$ is a vector field normal to $X^{a}$. For the covariant quantity $L_{\xi} X_{a}$ holds:

$$
L_{\xi} X_{a}=\left[(\ln X)_{, b} \xi^{b}+\psi+\frac{\varepsilon(X)}{X^{2}} H_{c b} X^{c} X^{b}\right] X_{a}+\hat{V}_{a}(X)
$$

in which

$$
\hat{V}_{a}(X)=2 h_{a}^{b}(X) H_{b c} X^{c}+V_{a}(X)
$$

Note that in general $\hat{V}_{a}(X) \neq V_{a}(X)$.

In case where $X^{a}$ is normalized, i.e., $X^{2}=1$ formulae (53) and (54) reduce to:

$$
\begin{aligned}
& L_{\xi} X^{a}=-\left[\psi+\varepsilon(X) H_{c b} X^{c} X^{b}\right] X^{a}+V^{a}(X) \\
& L_{\xi} X_{a}=\left[\psi+\varepsilon(X) H_{c b} X^{c} X^{b}\right] X_{a}+\hat{V}_{a}(X) .
\end{aligned}
$$

In the special case $X^{a}=u^{a}$, it follows

$$
\begin{aligned}
& L_{\xi} u^{a}=-\left(\psi-H_{c d} X^{c} X^{d}\right) u^{a}+V^{a}(u) \\
& L_{\xi} u_{a}=\left(\psi-H_{c d} X^{c} X^{d}\right) u_{a}+\hat{V}_{a}(u) .
\end{aligned}
$$

Some important results are collected in the following proposition.

Proposition 7.2 (i) $\hat{V}_{a}(X)=V^{a}(X)$ iff $h^{a b}(X) H_{b c} X^{c}=0$ iff $H_{b c} X^{c}=a X_{b}$ that is, $X^{a}$ is an eigenvector of $H_{b c}$.

(ii) $\hat{V}^{a}(X)=V^{a}(X)$ for all non-null $X^{a}$ iff $\xi^{a}$ is at most a $C K V$.

(iii) For $X^{a}=u^{a}$ we have $\hat{V}^{a}(u)=V^{a}(u)$ iff either $u^{a}$ is an eigenvector of $H_{b c}$ or $\xi^{a}$ is at most a $C K V$. Furthermore in that later case holds:

$$
\begin{aligned}
& L_{\xi} u^{a}=-\psi u^{a}+V^{a}(u) \\
& L_{\xi} u_{a}=\psi u_{a}+V_{a}(u) .
\end{aligned}
$$

These results are easily established and we omit the proof. 
7.1 The case $X^{2}=1$

In the following, we assume that $X^{a}$ is normalized, that is, $X^{a} X_{a}=\varepsilon(X)$, where $\varepsilon(X)= \pm 1$, and we compute the quantity $V_{a}(u)$ in terms of the "kinematic" quantities of $X^{a}$.

We find

$$
L_{\xi} X_{a}=\left[2 \omega(X)_{a b}+\varepsilon(X)\left(\stackrel{*}{X}_{a} X_{b}-\stackrel{*}{X}_{b} X_{a}\right)\right] \xi^{b}+\left(X_{b} \xi^{b}\right)_{; a}
$$

where a star “*” over a symbol indicates derivation along $X^{a}$, i.e., $\stackrel{*}{X}_{a}=X_{a ; b} X^{b}$.

We also write $2 \omega(X)_{a b}=h_{a}^{c}(X) h_{b}^{d}(X)\left(X_{c ; d}-X_{d ; c}\right)$. We have according to the general $1+3$ decomposition formula:

$$
X_{a ; b}=h(X)_{a}^{c} h(X)_{b}^{d} X_{a ; b}+\varepsilon(X) \stackrel{*}{X_{a}} X_{b} .
$$

We continue with the $1+3$ decomposition of $L_{\xi} X_{a}$ along $X^{a}$. For the parallel component, we find ${ }^{8}$ :

$$
X^{a} L_{\xi} X_{a}=-\left(\stackrel{*}{X_{b}} \xi^{b}\right)+\left(X_{b} \xi^{b}\right)^{*}=X_{b} \stackrel{*}{\xi}^{b} .
$$

This implies the formula ${ }^{9}$ :

$$
L_{\xi} X_{a}=\varepsilon(X)\left[-\left(\stackrel{*}{X}_{b} \xi^{b}\right)+\left(X_{b} \xi^{b}\right)^{*}\right]+\hat{V}_{a}(X) .
$$

By comparing (62) and (65), we find:

$$
\hat{V}_{a}(X)=2 \omega(X)_{a b} \xi^{b}+\varepsilon(X) \stackrel{*}{X}_{a}\left(X_{b} \xi^{b}\right)+\left(X_{b} \xi^{b}\right)_{; a}-\varepsilon(X)\left(X_{b} \xi^{b}\right)^{*} X_{a} .
$$

Noting that:

$$
\left(X_{b} \xi^{b}\right)_{; a}-\varepsilon(X)\left(X_{b} \xi^{b}\right)^{*} X_{a}=h_{a}^{b}\left(X_{c} \xi^{c}\right)_{; b},
$$

we obtain the final result:

$$
\hat{V}_{a}(X)=2 \omega(X)_{a b} \xi^{b}+\varepsilon(X) \stackrel{*}{X}_{a}\left(X_{b} \xi^{b}\right)+h_{a}^{b}\left(X_{c} \xi^{c}\right)_{; b} .
$$

It is possible to express the parallel component of the $L_{\xi} X_{a}$ in terms of the collineation components. Indeed by Lie differentiation of

$$
g^{a b} X_{a} X_{b}=\varepsilon(X)
$$

we find

$$
\begin{aligned}
\left(L_{\xi} g^{a b}\right) X_{a} X_{b}+2 g^{a b} X_{a} L_{\xi} X_{b} & =0 \Rightarrow \\
\left(-2 \psi g^{a b}-2 H^{a b}\right) X_{a} X_{b}+2 g^{a b} X_{a} L_{\xi} X_{b} & =0 \Rightarrow \\
X^{b} L_{\xi} X_{b} & =\varepsilon(X) \psi+H^{a b} X_{a} X_{b} .
\end{aligned}
$$

From (64) and (67) follows:

$$
\varepsilon(X) \psi+H^{a b} X_{a} X_{b}=-\left(\stackrel{*}{X_{b}} \xi^{b}\right)+\left(X_{b} \xi^{b}\right)^{*}=X_{b} \stackrel{*}{\xi}^{b} .
$$

This relation implies the identity/decomposition:

$$
L_{\xi} X_{a}=\left[\psi+\varepsilon(X) H^{c d} X_{c} X_{d}\right] X_{a}+2 \omega(X)_{a b} \xi^{b}+\varepsilon(X) \stackrel{*}{X_{a}}\left(X_{b} \xi^{b}\right)+h_{a}^{b}\left(X_{c} \xi^{c}\right)_{; b}
$$

8 We remark that $\stackrel{* b}{\xi}$ does not in general coincides with $\stackrel{*}{\xi}_{b}$.

${ }^{9}$ Compare with (57). Also note that $L_{\xi} X_{a}=\varepsilon(X)\left(X^{b} L_{\xi} X_{b}\right) X_{a}+h_{a}^{b} L_{\xi} X_{b}$. 
Next, we compute the Lie derivative $L_{\xi} X^{a}$. We find

$$
L_{\xi} X^{a}=\left[-\psi+\varepsilon(X) H^{c d} X_{c} X_{d}\right] X^{a}-2 H^{a b} X_{b}+2 \omega(X)_{. b}^{a} \xi^{b}+\varepsilon(X) X^{*}\left(X_{b} \xi^{b}\right)+h^{a b}\left(X_{c} \xi^{c}\right)_{; b} .
$$

But $\left(X^{2}=1\right)$ :

$$
H^{a b} X_{b}=\varepsilon(X)\left(H^{c d} X_{c} X_{d}\right) X^{a}+h_{c}^{a} H^{c d} X_{d}
$$

therefore,

$$
L_{\xi} X^{a}=\left[-\psi-\varepsilon(X) H^{c d} X_{c} X_{d}\right] X^{a}-2 h_{c}^{a} H^{c d} X_{d}+2 \omega(X)_{. b}^{a} \xi^{b}+\varepsilon(X) X^{*}\left(X_{b} \xi^{b}\right)+h^{a b}\left(X_{c} \xi^{c}\right)_{; b}
$$

Finally, by comparing with (57), we find the following constraint equation:

$$
V_{a}(X)=-2 h_{c}^{a} H^{c d} X_{d}+2 \omega(X)_{. b}^{a} \xi^{b}+\varepsilon(X) X^{*}\left(X_{b} \xi^{b}\right)+h^{a b}\left(X_{c} \xi^{c}\right)_{; b} .
$$

7.2 The special case $X^{a}=u^{a}$

An important special case is $X^{a}=u^{a}$, that is, $X^{a}$ is unit and timelike (the four-velocity). We write $L_{\xi} u_{a}$ and $L_{\xi} u^{a}$ given by formulae (58) and (59) in terms of the kinematic quantities of $u^{a}$.

From (58) follows:

$$
L_{\xi} u_{a}=\left[\psi-H^{c d} u_{c} u_{d}\right] u_{a}+2 \omega_{a b} \xi^{b}-\dot{u}_{a}\left(u_{b} \xi^{b}\right)+h_{a}^{b}\left(u_{c} \xi^{c}\right)_{; b}
$$

and from (59):

$$
L_{\xi} u^{a}=-\left[\psi-H^{c d} u_{c} u_{d}\right] u^{a}-2 h_{b}^{a} H^{b c} u_{c}+2 \omega_{. b}^{a} \xi^{b}-\dot{u}^{a}\left(u_{b} \xi^{b}\right)+h^{a b}\left(u_{c} \xi^{c}\right)_{; b} .
$$

These imply that

$$
\begin{aligned}
\hat{V}_{a}(u) & =2 \omega_{a b} \xi^{b}-\dot{u}_{a}\left(u_{b} \xi^{b}\right)+h_{a}^{b}\left(u_{c} \xi^{c}\right)_{; b} \\
V^{a}(u) & =-2 h_{b}^{a} H^{b c} u_{c}+2 \omega_{. b}^{a} \xi^{b}-\dot{u}^{a}\left(u_{b} \xi^{b}\right)+h^{a b}\left(u_{c} \xi^{c}\right)_{; b} .
\end{aligned}
$$

From (73), we draw the following conclusions:

1. If $u_{a} \xi^{a}$ is an acceleration potential (that is the acceleration potential is of the form $a=-\left(u_{b} \xi^{b}\right)^{-1}$ ) then for any collineation $\xi^{a}$ :

$$
L_{\xi} u_{a}=\left[\psi-H^{c d} u_{c} u_{d}\right] u_{a}+2 \omega_{a b} \xi^{b} .
$$
[12]).

One special case of this is when $u_{a} \xi^{a}=0$, i.e., the symmetry vector is normal to the flow vector $u^{a}$ (see

2. If $\xi^{a}$ is a $\mathrm{KV}$, we have

$$
L_{\xi} u_{a}=2 \omega_{a b} \xi^{b}-\dot{u}_{a}\left(u_{b} \xi^{b}\right)+h_{a}^{b}\left(u_{c} \xi^{c}\right)_{; b}
$$

from which we conclude

$$
L_{\xi} u_{a}=0 \Longleftrightarrow 2 \omega_{a b} \xi^{b}-\dot{u}_{a}\left(u_{b} \xi^{b}\right)+h_{a}^{b}\left(u_{c} \xi^{c}\right)_{; b}=0 .
$$

A CKV is inherited ${ }^{10}$ by the four-velocity field $u^{a}$ if $u_{b} \xi^{b}=0$ and the fluid is either irrotational (i.e., $\omega_{\alpha b}=0$ ) or the vorticity vector $\omega^{a} \| \xi^{a}$ (see [12]).

3. For a CKV, the inheritance of the symmetry by $u^{a}$ (i.e., $L_{\xi} u_{a}=\psi u_{a}$ or $L_{\xi} u^{a}=-\psi u^{a}$ ) is equivalent to the identity $h_{a}^{b} L_{\xi} \mathrm{d} x^{a}=0$ (see [20]). Obviously for a KV/HKV/SCKV this result remains true.

We note that the requirement of surface forming of $\xi^{a}$ with a unit timelike Killing vector (a static spacetime) results in the kinematic constraint (79) which is by no means trivial. For example, when $\xi^{a} u_{a}=0$ then $\omega_{a} \xi^{a}=0$, i.e., $\omega_{a} / / \xi^{a}$.

\footnotetext{
10 The symmetry is inherited if $L_{\xi} u^{a}=\lambda u^{a}$.
} 


\section{The $1+3$ decomposition of the Lie derivative $L_{\xi} X_{a ; b}$ wrt $X^{a}$}

This decomposition is useful because it allows us to compute the Lie derivative of the kinematic quantities along the collineation vector $\xi^{a}$. We have the following identity for any pair of vector fields ${ }^{11} X^{a}, \xi^{a}$ :

$$
L_{\xi} X_{a ; b}=\left(L_{\xi} X_{a}\right)_{; b}-\left(L_{\xi} \Gamma_{a b}^{c}\right) X_{c}
$$

and also the identity

$$
L_{\xi} \Gamma_{a b}^{c}=\frac{1}{2} g^{c d}\left[\left(L_{\xi} g_{d a}\right) ;_{b}+\left(L_{\xi} g_{d b}\right) ;{ }_{a}-\left(L_{\xi} g_{a b}\right) ; d\right] .
$$

Assuming $\xi^{a}$ to be a collineation, the second identity gives:

$$
L_{\xi} \Gamma_{a b}^{c}=g^{c d}\left[\psi, b g_{d a}+\psi,{ }_{a} g_{d b}-\psi, d g_{a b}+H_{d a ; b}+H_{d b ; a}-H_{a b ; d}\right] ;
$$

therefore,

$$
L_{\xi} X_{a ; b}=\left(L_{\xi} X_{a}\right)_{; b}-\left[\psi,{ }_{b} g_{d a}+\psi,{ }_{a} g_{d b}-\psi, d g_{a b}+H_{d a ; b}+H_{d b ; a}-H_{a b ; d}\right] X^{d} .
$$

A different expression is found as follows:

$$
\begin{aligned}
L_{\xi} \Gamma_{a b}^{c} & =\frac{1}{2} g^{c d}\left[\left(L_{\xi} g_{d a}\right)_{; b}+\left(L_{\xi} g_{d b}\right)_{; a}-\left(L_{\xi} g_{a b}\right) ; d\right] \\
& =g^{c d}\left[\left(\xi_{(d ; a) ; b}+\left(\xi_{(d ; b)}\right)_{a}-\left(\xi_{(a ; b)}\right) ; d\right]\right. \\
& =g^{c d}\left[\xi_{d ;(a b)}+\xi_{a ;[d b]}+\xi_{b ;[d a]}\right] \\
& =g^{c d}\left[\xi_{d ;(a b)}+\frac{1}{2} R_{t a d b} \xi^{t}+\frac{1}{2} R_{t b d a} \xi^{t}\right] \\
& =g^{c d}\left[\xi_{d ;(a b)}+R_{t(a|d| b)} \xi^{t}\right] .
\end{aligned}
$$

Therefore,

$$
L_{\xi} X_{a ; b}=\left(L_{\xi} X_{a}\right)_{; b}-g^{c d}\left[\xi_{d ;(a b)}+R_{t(a|d| b)} \xi^{t}\right] X_{c},
$$

where we have defined the Riemann tensor with the Ricci identity:

$$
2 \xi_{a ;[b c]}=R_{t a b c} \xi^{t} .
$$

8.1 The case $X^{2}=1$

We consider the case $X^{2}=1$ and find from (56)

$$
\begin{aligned}
\left(L_{\xi} X_{a}\right)_{; b} & \left.=\left[\psi+\varepsilon(X) H_{c d} X^{c} X^{d}\right] X_{a}+\hat{V}_{a}(X)\right] ; b \\
& =\left[\psi+\varepsilon(X) H_{c d} X^{c} X^{d}\right]_{; b} X_{a}+\left[\psi+\varepsilon(X) H_{c d} X^{c} X^{d}\right] X_{a ; b}+\hat{V}_{a}(X)_{; b},
\end{aligned}
$$

where

$$
\hat{V}_{a}(X)=2 \omega(X)_{a b} \xi^{b}+\varepsilon(X) X_{a}^{*}\left(X_{b} \xi^{b}\right)+h_{a}^{b}\left(X_{c} \xi^{c}\right)_{; b}
$$

To save writing, we set

$$
K(X)=\psi+\varepsilon(X) H_{c d} X^{c} X^{d}
$$

and have

$$
\left(L_{\xi} X_{a}\right)_{; b}=K(X)_{; b} X_{a}+K(X) X_{a ; b}+\hat{V}_{a}(X)_{; b}
$$

Then using (86), we get

$$
\begin{aligned}
L_{\xi} X_{a ; b} & =\left[K(X)_{; b} X_{a}+K(X) X_{a ; b}+\hat{V}_{a}(X)_{; b}\right] \\
& -\left[\psi, b g_{d a}+\psi, a g_{d b}-\psi, d g_{a b}+H_{d a ; b}+H_{d b ; a}-H_{a b ; d}\right] X^{d}
\end{aligned}
$$

and also

$$
L_{\xi} X_{a ; b}=\left[K(X)_{; b} X_{a}+K(X) X_{a ; b}+\hat{V}_{a}(X)_{; b}\right]-\left[\xi_{d ;(a b)}+R_{t(a|d| b)} \xi^{t}\right] X^{d} .
$$

11 This identity gives the commutation of the Lie and the covariant derivative in a Riemannian space. It can be found in [32]. 
8.2 The case $X^{a}$ is a unit timelike vector field, i.e., $X^{a}=u^{a}$

When $X^{a}=u^{a}$, from (89), we find

$$
\begin{aligned}
L_{\xi} u_{a ; b}= & K(u)_{; b} u_{a}+K(u) u_{a ; b}+\hat{V}_{a}(u)_{; b}+ \\
& -\left[\psi,{ }_{b} g_{d a}+\psi, a g_{d b}-\psi, d g_{a b}+H_{d a ; b}+H_{d b ; a}-H_{a b ; d}\right] u^{d},
\end{aligned}
$$

where

$$
K=\psi-H_{c d} u^{c} u^{d}, \hat{V}_{a}=\hat{V}_{a}(u) .
$$

We consider the $1+3$ decomposition $u_{a ; b}=\sigma_{a b}+\omega_{a b}+\frac{1}{2} \theta h_{a b}-\dot{u}_{a} u_{b}$ and the LHS of (91) becomes

$$
L_{\xi} \sigma_{a b}+L_{\xi} \omega_{a b}+\frac{1}{2} L_{\xi} \theta h_{a b}+\frac{1}{2} \theta L_{\xi} h_{a b}-\left(L_{\xi} \dot{u}_{a}\right) u_{b}-\dot{u}_{a} L_{\xi} u_{b} ;
$$

therefore, we can compute the quantities $L_{\xi} \sigma_{a b}, L_{\xi} \omega_{a b}, L_{\xi} \theta, L_{\xi} \dot{u}_{a}$ in terms of the collineation parameters $\psi, H_{a b}$ by taking the irreducible parts of Eq. (91). Before we do that we need to calculate the Lie derivative of the projection tensor $h_{a b}$.

\subsection{Calculation of $L_{\xi} h_{a b}$}

For the Lie derivative of the projection tensor, we find the results:

$$
\begin{aligned}
L_{\xi} h_{a b}(X)= & L_{\xi}\left(g_{a b}-\frac{\varepsilon(X)}{X^{2}} X_{a} X_{b}\right)=2 \psi h_{a b}(X)+2 H_{a b}-2 \frac{1}{X^{4}}\left(H_{c d} X^{c} X^{d}\right) X_{(a} X_{b)}+ \\
& -2 \frac{\varepsilon(X)}{X^{2}} X_{(a} \hat{V}_{b)}(X)-2 \frac{\varepsilon(X)}{X^{2}}\left[(\ln X)_{, c} \xi^{c}\right] X_{(a} X_{b)}, \\
L_{\xi} h_{b}^{a}(X) & =L_{\xi}\left(g_{b}^{a}-\frac{\varepsilon(X)}{X^{2}} X^{a} X_{b}\right) \\
& =-\frac{\varepsilon(X)}{X^{2}}\left[2(\ln X)_{, c} \xi^{c} X^{a} X_{b}+V^{a}(X) X_{b}+X^{a} \hat{V}_{b}(X)\right] .
\end{aligned}
$$

In the case where the vector field is unit $\left(X^{2}=1\right)$, Eqs. (94) and (95) reduce to

$$
\begin{aligned}
L_{\xi} h_{a b}(X) & =2 \psi h_{a b}(X)+2 H_{a b}-2\left(H_{c d} X^{c} X^{d}\right) X_{(a} X_{b)}-2 \varepsilon(X) X_{(a)} \hat{V}_{b)}(X) \\
L_{\xi} h_{b}^{a}(X) & =-\varepsilon(X)\left[V^{a}(X) X_{b}+X^{a} \hat{V}_{b}(X)\right] .
\end{aligned}
$$

In the special case where $X^{a}$ is the four-velocity $u^{a}$, the above relations become

$$
\begin{aligned}
L_{\xi} h_{a b} & =2 \psi h_{a b}+2 H_{a b}-2\left(H_{c d} u^{c} u^{d}\right) u_{(a} u_{b)}+2 u_{(a} \hat{V}_{b)} \\
L_{\xi} h_{b}^{a} & =u^{a} \hat{V}_{b}+V^{a} u_{b} .
\end{aligned}
$$

Replacing $\hat{V}_{b}, V^{a}$ from (75) and (76), we find

$$
\begin{aligned}
L_{\xi} h_{a b} & =2 \psi h_{a b}+2 H_{a b}-2\left(H_{c d} u^{c} u^{d}\right) u_{(a} u_{b)}+2 u_{(a}\left[2 \omega_{b)} \xi^{c}-\dot{u}_{b)}\left(u_{c} \xi^{c}\right)+h_{b)}^{d}\left(u_{c} \xi^{c}\right)_{; d}\right] \\
L_{\xi} h_{b}^{a} & =u^{a}\left[2 \omega_{b c} \xi^{c}-\dot{u}_{b}\left(u_{c} \xi^{c}\right)+h_{b}^{d}\left(u_{c} \xi^{c}\right)_{; d}\right]+\left[-2 h_{d}^{a} H^{d c} u_{c}+2 \omega_{. c}^{a} \xi^{c}-\dot{u}^{a}\left(u_{c} \xi^{c}\right)+h^{a d}\left(u_{c} \xi^{c}\right)_{; d}\right] u_{b} .
\end{aligned}
$$

Concerning $h^{a b}$, we find

$$
\begin{aligned}
L_{\xi} h^{a b} & =-g^{a c} g^{b d} L_{\xi} h_{c d} \\
& =-2 \psi h^{a b}-2 H^{a b}-g^{a c} g^{b d}\left[-2\left(H_{m n} u^{m} u^{n}\right) u_{c} u_{d}+2 u_{(c}\left[2 \omega_{d) m} \xi^{m}-\dot{u}_{d)}\left(u_{n} \xi^{n}\right)+h_{d)}^{m}\left(u_{n} \xi^{n}\right)_{; m}\right]\right] .
\end{aligned}
$$


Proposition 8.1 If $\xi^{a}$ is a spacelike $C K V$, the following formulae are true:

$$
\begin{aligned}
L_{\xi} h_{a b} & =2 \psi h_{a b}+4 u_{(a} \omega_{b)} \xi^{c}, \\
L_{\xi} h_{b}^{a} & =2\left(u^{a} \omega_{b c}+\omega^{a}{ }_{. c} u_{b}\right) \xi^{c}, \\
L_{\xi} h^{a b} & =-2 \psi h^{a b}-2 H^{a b}-4 g^{a c} g^{b d} u_{(c} \omega_{d) m} \xi^{m} .
\end{aligned}
$$

8.4 Calculation of $L_{\xi} \omega_{a b}, L_{\xi} \sigma_{a b}, L_{\xi} \theta, L_{\xi} \dot{u}_{a}$

We compute now the quantities $L_{\xi} \sigma_{a b}, L_{\xi} \omega_{a b}, L_{\xi} \theta, L_{\xi} \dot{u}_{a}$ in terms of the collineation parameters $\psi, H_{a b}$. We $\operatorname{have}^{12}(\hat{V}=\hat{V}(u), V=V(u))$ :

$$
\begin{aligned}
L_{\xi}\left(h_{a}^{c} h_{b}^{d} u_{c ; d}\right)= & h_{a}^{c} h_{b}^{d}\left(L_{\xi} u_{c ; d}\right)+h_{a}^{c}\left(L_{\xi} h_{b}^{d}\right) u_{c ; d}+h_{b}^{d}\left(L_{\xi} h_{a}^{c}\right) u_{c ; d} \\
= & h_{a}^{c} h_{b}^{d}\left[\left[\psi-H_{m n} u^{m} u^{n}\right] ; d u_{c}+\left[\psi-H_{m n} u^{m} u^{n}\right] u_{d ; c}+\hat{V}_{c ; d}\right] \\
& -h_{a}^{c} h_{b}^{d}\left[\psi, c g_{e d}+\psi, d g_{e c}-\psi, e g_{c d}+H_{e c ; d}+H_{e d ; c}-H_{c d ; e}\right] u^{e} \\
& +h_{a}^{c}\left[u^{d} \hat{V}_{b}+V^{d} u_{b}\right] u_{c ; d}+h_{b}^{d}\left[u^{c} \hat{V}_{a}+V^{c} u_{a}\right] u_{c ; d} \\
= & h_{a}^{c} h_{b}^{d}\left(\psi-H_{m n} u^{m} u^{n}\right) u_{c ; d}+h_{a}^{c} h_{b}^{d} \hat{V}_{c ; d} \\
& +h_{a}^{c} u_{b} u_{c ; d} V^{d}+h_{b}^{c} u_{a} u_{d ; c} V^{d}+\dot{u}_{a} \hat{V}_{b} \\
& +\dot{\psi} h_{a b}-h_{a}^{c} h_{b}^{d}\left[H_{e c ; d}+H_{e d ; c}-H_{c d ; e}\right] u^{e} .
\end{aligned}
$$

We consider the symmetric and the antisymmetric parts of $u_{a ; b}$. For the antisymmetric part, we find

$$
\begin{aligned}
& L_{\xi} \omega_{a b}=h_{a}^{c} h_{b}^{d} L_{\xi} u_{[c ; d]} \Rightarrow \\
& L_{\xi} \omega_{a b}=\left(\psi-H_{m n} u^{m} u^{n}\right) \omega_{a b}+h_{a}^{c} h_{b}^{d} \hat{V}_{[c ; d]}+\dot{u}_{[a} \hat{V}_{b]}-2 u_{[a} \omega_{b] c} \hat{V}^{c}
\end{aligned}
$$

For the symmetric part $\theta_{a b}=u_{(a ; b)}$, we find

$$
\begin{aligned}
L_{\xi} \theta_{a b}= & L_{\xi}\left(h_{a}^{c} h_{b}^{d} u_{(c ; d)}\right) \Rightarrow \\
L_{\xi} \theta_{a b}= & \left(\psi-H_{m n} u^{m} u^{n}\right) \theta_{a b}+h_{a}^{c} h_{b}^{d} \hat{V}_{(c ; d)}+2 u_{(a} \theta_{b) c} V^{c}+\dot{u}_{(a} \hat{V}_{b)} \\
& +\dot{\psi} h_{a b}-h_{(a}^{c} h_{b)}^{d}\left[H_{e c ; d}+H_{e d ; c}-H_{c d ; e}\right] u^{e} .
\end{aligned}
$$

We decompose $\theta_{a b}$ in trace and traceless part as follows:

$$
\theta_{a b}=\sigma_{a b}+\frac{1}{3} h_{a b} \theta .
$$

For the trace $\theta$, we find

$$
\begin{aligned}
& L_{\xi} \theta=-\left(\psi+H_{m n} u^{m} u^{n}\right) \theta-2 H^{a b} \theta_{a b}+h^{c d} \hat{V}_{(c ; d)}+2 \omega_{a b} \dot{u}^{a} \xi^{b}-\left(\dot{u}_{a} \dot{u}^{a}\right)\left(u_{b} \xi^{b}\right) \\
& +\dot{u}^{a}\left(u_{c} \xi^{c}\right)_{; a}+3 \dot{\psi}-h^{c d}\left[H_{e c ; d}+H_{e d ; c}-H_{c d ; e}\right] u^{e} .
\end{aligned}
$$

12 Recall that

$$
L_{\xi} u_{a}=\left[\psi-H^{c d} u_{c} u_{d}\right] u_{a}+2 \omega_{a b} \xi^{b}-\dot{u}_{a}\left(u_{b} \xi^{b}\right)+h_{a}^{b}\left(u_{c} \xi^{c}\right)_{; b}
$$

and from (70):

$$
L_{\xi} u^{a}=-\left[\psi+H^{c d} u_{c} u_{d}\right] u^{a}-2 h_{b}^{a} H^{b c} u_{c}+2 \omega_{. b}^{a} \xi^{b}-\dot{u}^{a}\left(u_{b} \xi^{b}\right)+h^{a b}\left(u_{c} \xi^{c}\right)_{; b},
$$

where

$$
\begin{aligned}
\hat{V}_{a}(u) & =2 \omega_{a b} \xi^{b}-\dot{u}_{a}\left(u_{b} \xi^{b}\right)+h_{a}^{b}\left(u_{c} \xi^{c}\right)_{; b} \\
V^{a}(u) & =-2 h_{b}^{a} H^{b c} u_{c}+2 \omega^{a}{ }_{b} \xi^{b}-\dot{u}^{a}\left(u_{b} \xi^{b}\right)+h^{a b}\left(u_{c} \xi^{c}\right)_{; b} .
\end{aligned}
$$


The term $2 H^{a b} \theta_{a b}$ becomes

$$
2 H^{a b} \theta_{a b}=2 H^{a b} \sigma_{a b}+\frac{2}{3} H^{a b} h_{a b} \theta=2 H^{a b} \sigma_{a b}+\frac{2}{3} H^{m n} u_{m} u_{n} \theta .
$$

Therefore,

$$
\begin{aligned}
L_{\xi} \theta= & -\left(\psi+\frac{5}{3} H_{m n} u^{m} u^{n}\right) \theta-2 H^{a b} \sigma_{a b}+h^{c d} \hat{V}_{(c ; d)}+2 \omega_{a b} \dot{u}^{a} \xi^{b} \\
& -\left(\dot{u}_{a} \dot{u}^{a}\right)\left(u_{b} \xi^{b}\right)+\dot{u}^{a}\left(u_{c} \xi^{c}\right)_{; a}+3 \dot{\psi}-h^{c d}\left[H_{e c ; d}+H_{e d ; c}-H_{c d ; e}\right] u^{e} .
\end{aligned}
$$

Concerning the four-acceleration $\dot{u}_{a}$, it follows

$$
L_{\xi} \dot{u}_{a}=\dot{K}(u) u_{a}+\hat{V}_{a}(u)_{; b} u^{b}+V^{b}(u) u_{a ; b}+\psi,_{a}-\left[H_{d a ; b}+H_{d b ; a}-H_{a b ; d}\right] u^{d} u^{b},
$$

where $K(u)=\psi-H_{m n} u^{m} u^{n}$.

Finally, for the shear $\sigma_{a b}$, we have

$$
\begin{aligned}
& L_{\xi} \sigma_{a b}=K(u) \sigma_{a b}-\frac{1}{3}\left(\frac{2}{3} H_{m n} u^{m} u^{n}\right) \theta h_{a b}+h_{a}^{c} h_{b}^{d} \hat{V}_{(c ; d)}+2 u_{(a} \theta_{b) c} V^{c}+\dot{u}_{(a} \hat{V}_{b)} \\
& -h_{(a}^{c} h_{b)}^{d}\left[H_{e c ; d}+H_{e d ; c}-H_{c d ; e}\right] u^{e}-\frac{1}{3}\left[-2 H_{a b}-2\left(H_{c d} u^{c} u^{d}\right) u_{(a} u_{b)}+2 u_{(a} \hat{V}_{b)}\right] \theta \\
& -\frac{1}{3}\left[\begin{array}{c}
-2 H^{m n} \sigma_{m n}+h^{m n} \hat{V}_{(m ; n)}+2 \omega_{m n} \dot{u}^{m} \xi^{n}-\left(\dot{u}_{m} \dot{u}^{m}\right)\left(u_{n} \xi^{n}\right) \\
+\dot{u}^{m}\left(u_{n} \xi^{n}\right)_{; m}-h^{m n}\left[H_{e m ; n}+H_{e n ; m}-H_{m n ; e}\right] u^{e}
\end{array}\right] h_{a b} .
\end{aligned}
$$

\section{The matter: dynamics}

General relativity studies the Physics in spacetime in the same way it is done in Special Relativity, that is, each physical field is described by a symmetric tensor $T_{a b}$ which is called the energy momentum tensor of the specific field. If the field is described by a Lagrangian $L$ then its energy momentum tensor in a model theory with metric tensor $g_{a b}$ is computed from the relation:

$$
T_{a b}=\frac{\delta L}{\delta g_{a b}} .
$$

In case in a given situation there are various fields interacting with the gravitational field, then the system of the fields is described by the sum of all the energy momentum tensors of all the fields involved, while the interaction with the gravitational field is described by the "conservation equation"

$$
\text { total } T_{; b}^{a b}=0 \text {. }
$$

Of course, in this approach, each field still has its own field equations which have to be satisfied in the model spacetime considered. For example, a scalar field $\phi$ given by the action integral

$$
S=\int L\left(g_{\mu \nu}, \phi, \phi ; k\right) \mathrm{d}^{4} x
$$

which leads to the Klein-Gordon equation:

$$
D_{i}\left(\frac{\partial L}{\partial \phi_{; i}}\right)-\frac{\partial L}{\partial \phi}=0
$$

In GR, the gravitational field is assumed to be created by all types of matter in spacetime. The energy momentum tensor of matter is the total energy momentum tensor total $T^{a b}$. In GR, it is also assumed that the totality of physical fields (including the gravitational field) formulate the geometry (that is the metric $g_{a b}$ ) of spacetime via Einstein field equations:

$$
G_{a b}=k T_{a b}
$$


where $G_{a b}$ is the Einstein tensor (of the particular $g_{a b}$ which is unknown!). The conservation equation (119) leads to the geometric condition $G_{; b}^{a b}=0$ which is the second Bianchi identity; hence, no new constraints are introduced. The constant $k$ is a universal constant because it relates two entities of a different nature (i.e., geometry and the physical fields) and it is called the Einstein gravitational constant. By choosing appropriate units (called physical units) one sets without loss of generality $k=1$. Furthermore, by demanding that GR agrees with the Newtonian theory in the solar system one relates $k$ to the Newtonian gravitational constant $G$ with the formula $k=\frac{8 \pi G}{c^{2}}$.

In this picture so far there is no observational status and we have a kind of theory which is universal for all observers and all types and combinations of matter. This is the scenario of general relativity [24]. We note that Einstein field equations (121) are independent of the observer and they relate the geometry of the background space (which is not specified!) with the matter content of the universe (which is also not specified). Therefore, they cannot be solved because one knows neither the metric, therefore, one cannot compute the $G_{a b}$, nor the matter, i.e., the energy momentum tensor $T_{a b}$. In other words Eq. (121) is used only to frame the general setup (i.e., the scenario) of the theory and cannot give any information until a class of observers and the matter content of spacetime are specified. This is the reason that GR is a theory of Physics which generates gravitational models instead of a theory giving a unique model, as it is the case with the Newtonian gravitational theory.

Before we consider the observational aspects of GR, that is, definite gravitational models as seen by a specified class of observers, we must address the more fundamental question, which is ${ }^{13}$

To what degree the matter content and the geometry of spacetime are interconnected as a result of the field equations (121)?

The answer to this question has two parts. The first is due to the inherent identities of the Riemannian geometry of spacetime and the second is the result of the assumed collineations of spacetime. Concerning the first we see that due to the contracted Bianchi identity (5) Einstein field equations (121) imply that $T_{a b}$ is symmetric $^{14}$ and that the conservation equations (119) are satisfied as a constraint/identity independently of the observers which are used to build a gravitational model.

Concerning the identities from the collineations assumed by a specific model we note that $L_{\xi} G_{a b}$ can be expressed in terms of the generic collineation $L_{\xi} g_{a b}$ and subsequently in terms of the collineation parameters $\psi, H_{a b}$. The general expression in terms of the trace and the traceless part is

$$
\begin{aligned}
L_{\xi} G_{a b}= & -(n-2) A_{a b}+2 K_{a b}-\square H_{a b}-R H_{a b} \\
& +\frac{1}{n} g_{a b}\left[(n-1)(n-2) \square \psi-(n-2) H_{. ; c d}^{c d}+n R_{c d} H^{c d}\right],
\end{aligned}
$$

where

$$
A_{a b}=\psi_{; a b}-\frac{1}{n} g_{a b} \square \psi \text { and } K_{a b}=H_{.(a ; b) d}^{d}-\frac{1}{n} g_{a b} H_{. ; c d}^{c d} .
$$

Then Einstein field equations (121) relate $L_{\xi} T_{a b}$ to the collineation parameters $\psi, H_{a b}$ which implies that the 'symmetries' of $T_{a b}$ as a two index tensor are related to the symmetries of the metric $g_{a b}$. In this manner, when we make a symmetry assumption in spacetime, we simultaneously impose a constraint in the form of a symmetry to the energy momentum tensor; therefore, we restrict the possible forms of matter this model spacetime can support. In a sense by an external assumption at the level of symmetry we constraint both the geometry and the matter content of spacetime.

To bring all the above at a level which can be used in practice, that is observation, we must introduce a class of observers $u^{a}$. The choice of these observers is our choice and there is no guarantee whatsoever that they are, or they will be, the intrinsic observers of spacetime. However, this is not to discourage us because it is the most we can do. The introduction of observers immediately introduces a kinematics in spacetime and all the considerations of the previous sections apply.

\footnotetext{
13 The question then is: Which class of observers?

The ideal choice would be the intrinsic observers but only 'God' knows who are they. What it is left to us is to choose an arbitrary class of observers by means of a timelike unit vector field and adding some more simplifying assumptions written down (121) in terms of some set of differential equations whose solutions will give us an indication of spacetime as seen by these observers. The experiment and the observations will show us how close to the intrinsic observers we are and how close to 'reality' our simplifying assumptions are. In conclusion, we cannot solve the field equations but we can use them as the vehicle to make up scientific pictures of the gravitational field and get some answers which we hope they will be as close as possible to our measurements (=reality!). The ultimate truth is hidden within the intrinsic observers with whom we have no touch or communication.

14 Or at least only the symmetric part of $T_{a b}$ enters the field equations and determines the gravitational field.
} 
Table 1 Types of energy-momentum tensors

\begin{tabular}{llllll}
\hline$\mu$ & $p$ & $q^{a}$ & $\pi^{a b}$ & $T_{a b}$ & Type of fluid \\
\hline 0 & 0 & 0 & 0 & 0 & Empty space \\
$\neq 0$ & 0 & 0 & 0 & $T_{a b}=\mu u_{a} u_{b}$ & Dust \\
$\neq 0$ & $\neq 0$ & 0 & 0 & $T_{a b}=\mu u_{a} u_{b}+p h_{a b}$ & Perfect fluid \\
$\neq 0$ & $\neq 0$ & $\neq 0$ & 0 & $T_{a b}=\mu u_{a} u_{b}+p h_{a b}+2 q_{(a} u_{b)}$ & Isotropic non-perfect fluid \\
$\neq 0$ & $\neq 0$ & 0 & $\neq 0$ & $T_{a b}=\mu u_{a} u_{b}+p h_{a b}+\pi_{a b}$ & Anisotropic fluid without heat flux \\
$\neq 0$ & $\neq 0$ & $\neq 0$ & $\neq 0$ & $T_{a b}=\mu u_{a} u_{b}+p h_{a b}+2 q_{(a} u_{b)}+\pi_{a b}$ & General anisotropic fluid \\
\hline
\end{tabular}

\section{The role of observers in GR}

As it has been remarked Einstein field equations provide the canvas on which the various gravitational models of GR are created. The construction of a gravitational model requires two necessary elements/actions:

a. Additional geometric assumptions which are made beyond the internal geometric identities of the Riemannian structure of spacetime.

b. The choice of a class of observers, that is, a timelike unit four vector field $u^{a}$ which defines the fourvelocity of the observers.

Note that in this scenario the energy momentum tensor $T_{a b}$ of matter and the other fields are not effected. What it changes is the $1+3$ decomposition of $T_{a b}$ in irreducible parts which encounter the observation by the specific observers of the physical variables corresponding to each irreducible part.

10.1 The $1+3$ decomposition of the energy momentum tensor: the dynamic variables

We apply the general formula (23) in the case $Y_{a b}=T_{a b}$, where $T_{a b}=T_{b a}$ is the energy momentum tensor. This defines the irreducible parts (tensors):

$$
\begin{aligned}
\mu & =T_{a b} u^{a} u^{b} \\
p & =\frac{1}{3} h^{a b} T_{a b} \\
q^{a} & =h^{a b} T_{b c} u^{c} \\
\pi_{a b} & =\left(h_{a}^{r} h_{b}^{s}-\frac{1}{3} h_{a b} h^{r s}\right) T_{r s}
\end{aligned}
$$

and we have the covariant decomposition/identity:

$$
T_{a b}=\mu u_{a} u_{b}+p h_{a b}+2 q_{(a} u_{b)}+\pi_{a b} .
$$

We note that in this decomposition $T_{a b}$ is described by two scalar fields $(\mu, p)$, one spacelike vector $\left(q^{a}, q_{a} u^{a}=0\right)$ and a traceless symmetric 2-tensor $\left(\pi_{a b}, g_{a b} \pi^{a b}=0\right)$.

These quantities we call the physical variables and assume that they represent the mass density, the isotropic pressure, the heat flux and the traceless stress tensor, respectively, as measured by the observers $u^{a}$. Due to this decomposition and the assumed physical interpretation, we consider the types of 'gravitating fluids' given in Table 1 .

10.2 The $1+3$ decomposition of the conservation equations

The conservation equations follow from the contracted Bianchi identity and Einstein field equations. By $1+3$ decomposing $T_{; b}^{a b}=0$ wrt $u^{a}$ we find the conservation equations as two sets of equations, one set resulting from the projection along the direction $u^{a}$ and one normal to $u^{a}$. We replace $T^{a b}$ from (128) in terms of the physical variables and find the two conservation equations ${ }^{15}$ :

$$
\dot{\mu}+(\mu+p) \theta+q_{; b}^{b}+q^{a} \dot{u}_{a}+\pi^{a b} \sigma_{a b}=0 .
$$

\footnotetext{
15 We write as usually the derivative along $u^{a}$ by a dot, e.g. $\dot{A}^{a}=A_{; b}^{a} u^{b}$.
} 


$$
(\mu+p) \dot{u}_{a}+h_{a}^{c}\left(p_{, c}+\dot{q}_{c}+\pi_{c ; d}^{d}\right)+q^{c}\left(\omega_{a c}+\sigma_{a c}+\frac{4}{3} \theta h_{a c}\right)=0
$$

\section{The physical role of the propagation and the constraint equations}

The propagation and the constraint equations have been written in terms of the kinematic variables and the Ricci tensor. However, with the introduction of the physical variables by the $1+3$ decomposition of the energy momentum tensor we can use Einstein equations and replace $R_{a b}$ in terms of the physical variables. Then we have the complete physical role of the propagation and the constraint equations.

Einstein field equations provide

$$
R_{a b}=T_{a b}-\frac{1}{2} g_{a b} T+\Lambda g_{a b}
$$

where $T_{a b}=\mu u_{a} u_{b}+p h_{a b}+2 q_{(a} u_{b)}+\pi_{a b}$ by (128) and the trace:

$$
T=T_{a}^{a}=-\mu+3 p .
$$

Replacing in (131) we find $R_{a b}$ in terms of the physical variables:

$$
R_{a b}=\mu u_{a} u_{b}+p h_{a b}+2 q_{(a} u_{b)}+\pi_{a b}+\frac{1}{2} g_{a b}(\mu-3 p+2 \Lambda)
$$

or

$$
R_{a b}=(\mu+p) u_{a} u_{b}+\frac{1}{2}(\mu-p+2 \Lambda) g_{a b}+2 q_{(a} u_{b)}+\pi_{a b}
$$

From (133) follows:

$$
\begin{aligned}
R_{a b} u^{a} u^{b} & =\mu-\frac{1}{2}(\mu-3 p)-\Lambda=\frac{1}{2} \mu+\frac{3}{2} p-\Lambda \\
R_{a b} u^{a} & =-\mu u_{b}-\frac{1}{2} q_{b}+\frac{1}{2} u_{b}(\mu-3 p+2 \Lambda) \\
R & =R_{a}^{a}=\mu-3 p+4 \Lambda,
\end{aligned}
$$

where $R=g^{a b} R_{a b}$ is the curvature scalar.

We consider now the constraint and propagation equations and we divide them in two sets. One set contains the ones which do not contain the curvature tensor and the Ricci tensor; therefore, they are independent of the field equations (and consequently the matter content of the universe) and they are purely kinematic equations. The second set contains the equations which depend on the Ricci tensor and the field equations and are the dynamical propagation and constraint equations.

11.1 The dynamical propagation and constraint equations

The dynamical propagation equations ${ }^{16}$ are Eqs. (38) and (39). Replacing the Ricci tensor from (133) we find for the first:

$$
\dot{\theta}+\frac{1}{3} \theta^{2}+2\left(\sigma^{2}-\omega^{2}\right)-\dot{u}_{; a}^{a}=-\frac{1}{2}(\mu+3 p)+\Lambda .
$$

This is known as theRaychaudhuri equation.

Concerning the propagation equation (39), we have

$$
h_{s}^{a} h_{t}^{b}\left[\dot{\sigma}_{a b}-\dot{u}_{(a ; b)}\right]+\sigma_{(s|c|} \sigma_{t)}^{c}+\frac{2}{3} \sigma_{s t} \theta+\omega_{s} \omega_{t}-\dot{u}_{s} \dot{u}_{t}-\frac{1}{3}\left(2 \sigma^{2}+\omega^{2}-\dot{u}_{; b}^{b}\right) h_{s t}
$$

\footnotetext{
16 The remaining propagation equation (37) giving the propagation of the vorticity does not involve the Ricci tensor; hence, it is a kinematical equation.
} 


$$
\begin{aligned}
& =-E_{s t}+\frac{1}{2}\left(h_{s}^{a} h_{t}^{b}-\frac{1}{3} h_{s t} h^{a b}\right) R_{a b} \\
& =-E_{s t}+\frac{1}{2}\left(h_{s}^{a} h_{t}^{b}-\frac{1}{3} h_{s t} h^{a b}\right)\left(\mu u_{a} u_{b}+p h_{a b}+2 q_{(a} u_{b)}+\pi_{a b}+\frac{1}{2} g_{a b}(\mu-3 p+2 \Lambda)\right) \\
& =-E_{s t}+\frac{1}{2}\left(h_{s}^{a} h_{t}^{b}-\frac{1}{3} h_{s t} h^{a b}\right) \pi_{a b}=-E_{s t}+\frac{1}{2} \pi_{s t} .
\end{aligned}
$$

Therefore in terms of the physical variables, the propagation equation (39) reads:

$$
h_{s}^{a} h_{t}^{b}\left[\dot{\sigma}_{a b}-\dot{u}_{(a ; b)}\right]+\sigma_{(s|c|} \sigma_{t)}^{c}+\frac{2}{3} \sigma_{s t} \theta+\omega_{s} \omega_{t}-\dot{u}_{s} \dot{u}_{t}-\frac{1}{3}\left(2 \sigma^{2}+\omega^{2}-\dot{u}_{; b}^{b}\right) h_{s t}=-E_{s t}+\frac{1}{2} \pi_{s t} .
$$

This equation gives the propagation of shear.

Moreover, the only dynamic constraint equation is equation (41). We find

$$
h_{s}^{c}\left[\frac{2}{3} \theta,{ }_{c}-h^{a b} \sigma_{a c ; b}-\eta_{c a m n} u^{a}\left(\omega^{m ; n}+2 \omega^{m} \dot{u}^{n}\right)\right]=q_{b} .
$$

\section{The dynamic role of collineations}

As we remarked in Sect. 9 the collineation parameters restrict the possible forms of $T_{a b}$. Indeed Einstein field equations and (122) give

$$
\begin{aligned}
L_{\xi} T_{a b}= & -(n-2) A_{a b}+2 K_{a b}-\square H_{a b}-R H_{a b} \\
& +\frac{1}{n} g_{a b}\left[(n-1)(n-2) \square \psi-(n-2) H_{. ; c d}^{c d}+n R_{c d} H^{c d}\right],
\end{aligned}
$$

where the tensors $A_{a b}, K_{a b}$ are given in (123). From this equation, it is possible to give the effect of a collineation directly to the physical parameters of the observers $u^{a}$. Indeed if we replace in (141) $T_{a b}$ in $L_{\xi} T_{a b}$ using (128) and take the irreducible parts, we express the Lie derivative along the collineation vector of the dynamical variables in terms of the collineation parameters. This form of the field equations will relate directly the physical and the geometric variables thus enabling one to draw direct conclusions of the symmetry assumptions at the level of dynamics. Furthermore, the approach is completely general and the results hold for all types of matter, all observers and all collineations.

From (134) we decompose the $L_{\xi} R_{a b}$ in irreducible parts with respect to the $1+3$ decomposition defined by $u^{a}$ :

$$
\begin{aligned}
L_{\xi} R_{a b}= & {\left[\frac{1}{2}(\mu+3 p)^{\circ}-2 \stackrel{\circ}{q}_{c} u^{c}+\stackrel{\circ}{\pi}_{c d} u^{c} u^{d}-(\mu+3 p-2 \Lambda)\left(\dot{\xi}_{c} u^{c}\right)-2 q_{c} \dot{\xi}^{c}\right] u_{a} u_{b} } \\
& -2\left[\begin{array}{c}
\left.-(\mu+p) \stackrel{\circ}{u}_{d} h_{c}^{d}-\stackrel{\circ}{q}_{d} h_{c}^{d}+\stackrel{\circ}{\pi}_{d e} u^{d} h_{c}^{e}-\frac{1}{2}(\mu+3 p-2 \Lambda) u_{c} \xi_{; d}^{c} h_{e}^{d}\right] u_{(a} h_{e)}^{c}+ \\
-q_{c} \xi_{; d}^{c} h_{e}^{d}+\frac{1}{2}(\mu-p+2 \Lambda) \dot{\xi}_{d} h_{e}^{d}+\left(q_{d} h_{e}^{d}\right)\left(\dot{\xi}^{c} u_{c}\right)-h_{e}^{d} \pi_{c d} \dot{\xi}^{c}
\end{array}\right] \\
& +2\left[\frac{3}{2}(\mu-p)^{\circ}+2 q_{c} \grave{u}^{c}+\stackrel{\circ}{\pi}_{c d} h^{c d}+(\mu-p+2 \Lambda)\left(4 \psi+\dot{\xi}^{c} u_{c}\right)+2 u_{c} \xi_{; d}^{c} q^{d}+2 \pi_{c d} H^{c d}\right] h_{a b} \\
& +2\left[\begin{array}{c}
\frac{1}{2}(\mu-p)^{\circ} h_{e f}+2 q_{(c} \stackrel{\circ}{u}_{d)} h_{e}^{c} h_{f}^{d}+\stackrel{\circ}{\pi}_{c d} h_{e}^{c} h_{f}^{d} \\
+(\mu-p+2 \Lambda) \xi_{d ; r} h_{e}^{d} h_{f}^{r}+2 q_{r} u_{c} \xi_{; d}^{c} h_{e}^{d} h_{f}^{r}+2 \pi_{c r} \xi_{; d}^{c} h_{e}^{d} h_{f}^{r}
\end{array}\right]\left[h_{(a}^{e} h_{b)}^{f}-\frac{1}{3} h_{a b} h^{e f}\right]
\end{aligned}
$$

where $\circ$ means covariant derivation along $\xi^{a}$ e.g. $\stackrel{\AA}{\mu}=\mu_{; a} \xi^{a}$.Also from (16) we have (for $n=4$ !):

$$
L_{\xi} R_{a b}=-2 \psi_{; a b}-g_{a b} \square \psi+2 H_{.(a ; b) d}^{d}-\square H_{a b} .
$$


We $1+3$ decompose this expression and equate with (142) from which we find the field equations in the form of Lie derivative of physical variables in terms of kinematic variables and the collineation parameters.

To do that, we $1+3$ decompose first $\psi_{; a b}$. We write

$$
\psi_{; a b}=\lambda_{\psi} u_{a} u_{b}+p_{\psi} h_{a b}+2 q_{\psi(a} u_{b)}+\pi_{\psi a b},
$$

where

$$
\mu_{\psi}=\psi_{; a b} u^{a} u^{b}, p_{\psi}=\frac{1}{3} \psi_{; a b} h^{a b}, q_{\psi a}=-\psi_{; b c} h_{a}^{b} u^{c}, \pi_{\psi a b}=\left(h_{a}^{r} h_{b}^{s}-\frac{1}{3} h_{a b} h^{r s}\right) \psi_{; r s} .
$$

We also have

$$
\square \psi=\psi_{; a b} g^{a b}=-\mu_{\psi}+3 p_{\psi} .
$$

It is also possible to $1+3$ decompose the collineation tensor $H_{a b}$ as follows:

$$
H_{a b}=\mu_{H} u_{a} u_{b}+p_{H} h_{a b}-2 q_{H(a} u_{b)}+\pi_{H a b},
$$

where

$$
\mu_{H}=H_{a b} u^{a} u^{b}, p_{H}=\frac{1}{3} H_{a b} h^{a b}, q_{H a}=-H_{b c} h_{a}^{b} u^{c}, \pi_{H a b}=\left(h_{a}^{r} h_{b}^{s}-\frac{1}{3} h_{a b} h^{r s}\right) H_{r s} .
$$

We compute

$$
\begin{aligned}
-2 \psi_{; a b}-g_{a b} \square \psi & =-2\left(\mu_{\psi} u_{a} u_{b}+p_{\psi} h_{a b}+2 q_{\psi(a} u_{b)}+\pi_{\psi a b}\right)+\left(\mu_{\psi}-3 p_{\psi}\right)\left(-u_{a} u_{b}+h_{a b}\right) \\
& =-3\left(\mu_{\psi}-p_{\psi}\right) u_{a} u_{b}+\left(\mu_{\psi}-5 p_{\psi}\right) h_{a b}-4 q_{\psi(a} u_{b)}-2 \pi_{\psi a b} .
\end{aligned}
$$

Concerning the second term in the RHS of (143), we define

$$
K_{a b}=2 H_{.(a ; b) d}^{d}-\square H_{a b}
$$

and we write

$$
K_{a b}=\mu_{K} u_{a} u_{b}+p_{K} h_{a b}-2 q_{K(a} u_{b)}+\pi_{K a b},
$$

where

$$
\mu_{K}=K_{a b} u^{a} u^{b}, p_{K}=\frac{1}{3} K_{a b} h^{a b}, q_{K a}=-K_{b c} h_{a}^{b} u^{c}, \pi_{K a b}=\left(h_{a}^{r} h_{b}^{s}-\frac{1}{3} h_{a b} h^{r s}\right) K_{r s} .
$$

Then we have

$$
\begin{aligned}
L_{\xi} R_{a b}= & \left(-3 \mu_{\psi}+3 p_{\psi}+\mu_{K}\right) u_{a} u_{b}+\left(\mu_{\psi}-5 p_{\psi}+p_{K}\right) h_{a b} \\
& +2\left(-2 q_{\psi(a}+q_{K(a}\right) u_{b)}-2 \pi_{\psi a b}+\pi_{K a b} .
\end{aligned}
$$

Comparing with (142), we write the field equations in the following form:

$$
\begin{aligned}
& \frac{1}{2}(\mu+3 p)^{\circ}-2 \stackrel{\circ}{q}_{c} u^{c}+\stackrel{\circ}{\pi}_{c d} u^{c} u^{d}-(\mu+3 p-2 \Lambda)\left(\dot{\xi}_{c} u^{c}\right)-2 q_{c} \dot{\xi}^{c}=-3 \mu_{\psi}+3 p_{\psi}+\mu_{K}, \\
& {\left[\begin{array}{c}
-(\mu+p) \stackrel{\circ}{u}_{d} h_{e}^{d}-\stackrel{\circ}{q}_{d} h_{e}^{d}+\stackrel{\circ}{\pi}_{d c} u^{d} h_{e}^{c}-\frac{1}{2}(\mu+3 p-2 \Lambda) u_{c} \xi_{; d}^{c} h_{e}^{d} \\
-q_{c} \xi_{; d}^{c} h_{e}^{d}+\frac{1}{2}(\mu-p+2 \Lambda) \dot{\xi}_{d} h_{e}^{d}+\left(q_{d} h_{e}^{d}\right)\left(\dot{\xi}^{c} u_{c}\right)-h_{e}^{d} \pi_{c d} \dot{\xi}^{c}
\end{array}\right]=-\left(-2 q_{\psi e}+q_{K e}\right),}
\end{aligned}
$$




$$
\begin{aligned}
& \quad \frac{3}{2}(\mu-p)^{\circ}+2 q_{c} \stackrel{\circ}{ }^{c}+\stackrel{\circ}{\pi}_{c d} h^{c d}+(\mu-p+2 \Lambda)\left(4 \psi+\dot{\xi}^{c} u_{c}\right)+2 u_{c} \xi_{; d}^{c} q^{d}+2 \pi_{c d} H^{c d} \\
& =\frac{3}{2}\left(\mu_{\psi}-5 p_{\psi}+p_{K}\right), \\
& 2\left[\begin{array}{c}
\frac{1}{2}(\mu-p)^{\circ} h_{e f}+2 q_{(c} \stackrel{\circ}{u}_{d} h_{e}^{c} h_{f}^{d}+\stackrel{\circ}{\pi}_{c d} h_{e}^{c} h_{f}^{d} \\
+(\mu-p+2 \Lambda) \xi_{d ; r} h_{e}^{d} h_{f}^{r}+2 q_{r} u_{c} \xi_{; d}^{c} h_{e}^{d} h_{f}^{r}+2 \pi_{c r} \xi_{; d}^{c} h_{e}^{d} h_{f}^{r}
\end{array}\right] \quad\left[h_{(a)}^{e} h_{b)}^{f}-\frac{1}{3} h_{a b} h^{e f}\right] \\
& =-2 \pi_{\psi a b}+\pi_{K a b} .
\end{aligned}
$$

It is possible to simplify these equations.

We note the relations:

$$
\begin{aligned}
\dot{\xi}^{c} u_{c} & =\xi_{c ; d} u^{c} u^{d}=-\psi+H_{c d} u^{c} u^{d} \\
q^{d}\left(u_{c} \xi_{; d}^{c}+\dot{\xi}_{d}\right) & =q^{d}\left(u^{c} \xi_{c ; d}+\xi_{d ; c} u^{c}\right)=2 q^{d} u^{c} H_{c d}
\end{aligned}
$$

Then Eq. (153) is written as follows:

$$
\begin{aligned}
\text { LHS } & =\frac{1}{2}\left\{(\mu+3 p)^{\circ}-4 \stackrel{\circ}{q}_{c} u^{c}+2 \stackrel{\circ}{\pi}_{c d} u^{c} u^{d}-2(\mu+3 p-2 \Lambda)\left(\dot{\xi}_{c} u^{c}\right)-4 q_{c} \dot{\xi}^{c}\right\} \\
& =\frac{1}{2}\left\{(\mu+3 p)^{\circ}+4 q_{c} \stackrel{\circ}{ }^{c}+2 \stackrel{\circ}{\pi}_{c d} u^{c} u^{d}-2(\mu+3 p-2 \Lambda)\left(-\psi+H_{c d} u^{c} u^{d}\right)-4 q_{c} \dot{\xi}^{c}\right\} \\
& =\frac{1}{2}\left\{(\mu+3 p)^{\circ}+4 q_{c}\left(\stackrel{\circ}{ }^{c}-\dot{\xi}^{c}\right)+2 \stackrel{\circ}{\pi}_{c d} u^{c} u^{d}-2(\mu+3 p-2 \Lambda)\left(-\psi+H_{c d} u^{c} u^{d}\right)\right\} \\
& =\frac{1}{2}\left\{(\mu+3 p)^{\circ}+4 q_{c} L_{\xi} u^{c}+2 \stackrel{\circ}{\pi}_{c d} u^{c} u^{d}-2(\mu+3 p-2 \Lambda)\left(-\psi+H_{c d} u^{c} u^{d}\right)\right\} ;
\end{aligned}
$$

hence, (153) becomes

$$
\begin{aligned}
& (\mu+3 p)^{\circ}+4 q_{c} L_{\xi} u^{c}+2 \stackrel{\circ}{\pi}_{c d} u^{c} u^{d}-2(\mu+3 p-2 \Lambda) \\
& \left(-\psi+H_{c d} u^{c} u^{d}\right)=2\left(-3 \mu_{\psi}+3 p_{\psi}+\mu_{K}\right) .
\end{aligned}
$$

Similarly, for expression (155), we have

\section{LHS}

$$
\begin{aligned}
= & 2\left[\frac{3}{2}(\mu-p)^{\circ}+2 q_{c} \stackrel{\circ}{ }^{c}+\stackrel{\circ}{\pi}_{c d} h^{c d}+(\mu-p+2 \Lambda)\left(4 \psi+\dot{\xi}^{c} u_{c}\right)+2 u_{c} \xi_{; d}^{c} q^{d}+2 \pi_{c d} H^{c d}\right] \\
= & 2\left[\frac{3}{2}(\mu-p)^{\circ}+2 q_{c} \stackrel{\circ}{ }^{c}+\stackrel{\circ}{\pi}_{c d} u^{c} u^{d}+(\mu-p+2 \Lambda)\left(3 \psi+H_{c d} u^{c} u^{d}\right)\right. \\
& \left.+2 u^{c} q^{d}\left(\psi g_{c d}+H_{c d}-\xi_{d ; c}\right)+2 \pi_{c d} H^{c d}\right] \\
= & 2\left[\frac{3}{2}(\mu-p)^{\circ}+2 q_{c}\left(\stackrel{\circ}{u}^{c}-\dot{\xi}^{c}\right)+\stackrel{\circ}{\pi}_{c d} u^{c} u^{d}+(\mu-p+2 \Lambda)\left(3 \psi+H_{c d} u^{c} u^{d}\right)+2 u^{c} q^{d} H_{c d}+2 \pi_{c d} H^{c d}\right] \\
= & 3(\mu-p)^{\circ}+4 q_{c} L_{\xi} u^{c}+2 \stackrel{\circ}{\pi}_{c d} u^{c} u^{d}+2(\mu-p+2 \Lambda)\left(3 \psi+H_{c d} u^{c} u^{d}\right)+42 u^{c} q^{d} H_{c d}+4 \pi_{c d} H^{c d},
\end{aligned}
$$

that is,

$$
\begin{aligned}
& 3(\mu-p)^{\circ}+4 q_{c} L_{\xi} u^{c}+2 \stackrel{\circ}{\pi}_{c d} u^{c} u^{d}+2(\mu-p+2 \Lambda)\left(3 \psi+H_{c d} u^{c} u^{d}\right)+4 H_{c d} u^{c} q^{d}+4 \pi_{c d} H^{c d} \\
& =3\left(\mu_{\psi}-5 p_{\psi}+p_{K}\right)
\end{aligned}
$$

Adding the two new equations we get:

$$
\begin{aligned}
\text { LHS }= & (\mu+3 p)^{\circ}+3(\mu-p)^{\circ}+8 q_{c} L_{\xi} u^{c}+4 \stackrel{\circ}{\pi}_{c d} u^{c} u^{d} \\
& +2(3 \mu-3 p+6 \Lambda+\mu+3 p-2 \Lambda) \psi
\end{aligned}
$$




$$
\begin{aligned}
& +2(\mu-p+2 \Lambda-\mu-3 p+2 \Lambda) H_{c d} u^{c} u^{d} \\
& +4 H_{c d} u^{c} q^{d}+4 \pi_{c d} H^{c d} \\
= & 4 \dot{\mu}+8 q_{c} L_{\xi} u^{c}+4 \dot{\pi}_{c d} u^{c} u^{d}+8(\mu+\Lambda) \psi \\
& +8(-p+\Lambda) H_{c d} u^{c} u^{d}+4 H_{c d} u^{c} q^{d}+4 \pi_{c d} H^{c d}
\end{aligned}
$$

$$
\mathrm{RHS}=3\left(\mu_{\psi}-5 p_{\psi}+p_{K}\right)+2\left(-3 \mu_{\psi}+3 p_{\psi}+\mu_{K}\right)=-3 \mu_{\psi}-9 p_{\psi}+3 p_{K}+2 \mu_{K},
$$

that is,

$$
\begin{aligned}
4 \dot{\mu} & +8 q_{c} L_{\xi} u^{c}+4 \stackrel{\circ}{\pi}_{c d} u^{c} u^{d}+8(\mu+\Lambda) \psi \\
& +8(-p+\Lambda) H_{c d} u^{c} u^{d}+4 H_{c d} u^{c} q^{d}+4 \pi_{c d} H^{c d} \\
& =-3 \mu_{\psi}-9 p_{\psi}+3 p_{K}+2 \mu_{K}
\end{aligned}
$$

or

$$
\begin{aligned}
\stackrel{\circ}{\mu}= & \frac{1}{4}\left(-3 \mu_{\psi}-9 p_{\psi}+3 p_{K}+2 \mu_{K}\right)-2 q_{c} L_{\xi} u^{c}-\stackrel{\circ}{\pi}_{c d} u^{c} u^{d}-2(\mu+\Lambda) \psi \\
& +2(p-\Lambda) H_{c d} u^{c} u^{d}-H_{c d} u^{c} q^{d}-\pi_{c d} H^{c d} .
\end{aligned}
$$

The last equation expresses the derivative of $\mu$ along the collineation vector $\xi^{a}$. To find $\stackrel{\circ}{p}$ we use (157) and replace $\dot{\mu}$. We have

$$
\begin{aligned}
& 3 \stackrel{\circ}{p}+\frac{1}{4}\left(-3 \mu_{\psi}-9 p_{\psi}+3 p_{K}+2 \mu_{K}\right)-2(\mu+\Lambda) \psi \\
& +2(p-\Lambda) H_{c d} u^{c} u^{d}-H_{c d} u^{c} q^{d}-\pi_{c d} H^{c d} \\
& +2 q_{c} L_{\xi} u^{c}+\stackrel{\circ}{\pi}_{c d} u^{c} u^{d}-2(\mu+3 p-2 \Lambda)\left(-\psi+H_{c d} u^{c} u^{d}\right) \\
& =2\left(-3 \mu_{\psi}+3 p_{\psi}+\mu_{K}\right) \\
& 3 \stackrel{p}{p}-H_{c d} u^{c} q^{d}-\pi_{c d} H^{c d} \\
& +2 q_{c} L_{\xi} u^{c}+\AA_{\pi} u^{c} u^{d}+\frac{1}{4}\left(-3 \mu_{\psi}-9 p_{\psi}+3 p_{K}+2 \mu_{K}\right) \\
& +2(\mu+3 p-2 \Lambda-\mu-\Lambda) \psi+2(p-\Lambda-\mu-3 p+2 \Lambda) H_{c d} u^{c} u^{d} \\
& =2\left(-3 \mu_{\psi}+3 p_{\psi}+\mu_{K}\right) \\
& 3 \stackrel{\circ}{p}-H_{c d} u^{c} q^{d}-\pi_{c d} H^{c d} \\
& +2 q_{c} L \xi u^{c}+\stackrel{\circ}{\pi}_{c d} u^{c} u^{d} \\
& +6(p-\Lambda) \psi-2(2 p+\mu-\Lambda) H_{c d} u^{c} u^{d} \\
& =2\left(-3 \mu_{\psi}+3 p_{\psi}+\mu_{K}\right)-\frac{1}{4}\left(-3 \mu_{\psi}-9 p_{\psi}+3 p_{K}+2 \mu_{K}\right) \\
& 3 \stackrel{\circ}{p}-H_{c d} u^{c} q^{d}-\pi_{c d} H^{c d}+2 q_{c} L_{\xi} u^{c}+\stackrel{\circ}{\pi}_{c d} u^{c} u^{d} \\
& +6(p-\Lambda) \psi-2(2 p+\mu-\Lambda) H_{c d} u^{c} u^{d} \\
& =\frac{3}{4}\left(7 \mu_{\psi}+11 p_{\psi}+2 \mu_{K}-p_{K}\right) \\
& 3 \stackrel{\circ}{p}=H_{c d} u^{c} q^{d}+\pi_{c d} H^{c d}-2 q_{c} L_{\xi} u^{c}-\stackrel{\circ}{\pi}_{c d} u^{c} u^{d} \\
& -6(p-\Lambda) \psi+2(2 p+\mu-\Lambda) H_{c d} u^{c} u^{d} \\
& +\frac{1}{4}\left(-3 \mu_{\psi}-9 p_{\psi}+3 p_{K}+2 \mu_{K}\right) \text {. }
\end{aligned}
$$


We concentrate now on (154). We have

$$
\begin{aligned}
\text { LHS }= & -(\mu+p) \stackrel{\circ}{d}_{d} h_{e}^{d}-\stackrel{\circ}{q}_{d} h_{e}^{d}+\stackrel{\circ}{\pi}_{d c} u^{d} h_{e}^{c}-\frac{1}{2}(\mu+3 p-2 \Lambda) u_{c} \xi_{; d}^{c} h_{e}^{d} \\
& -q_{c} \xi_{; d}^{c} h_{e}^{d}+\frac{1}{2}(\mu-p+2 \Lambda) \dot{\xi}_{d} h_{e}^{d}+\left(q_{d} h_{e}^{d}\right)\left(\dot{\xi}^{c} u_{c}\right)-h_{e}^{d} \pi_{c d} \dot{\xi}^{c}
\end{aligned}
$$

The term

$$
\begin{aligned}
- & \frac{1}{2}(\mu+3 p-2 \Lambda) u_{c} \xi_{; d}^{c} h_{e}^{d}+\frac{1}{2}(\mu-p+2 \Lambda) \dot{\xi}_{d} h_{e}^{d} \\
& =-\frac{1}{2}(\mu+3 p-2 \Lambda) H_{c d} u^{c} h_{e}^{d}+\frac{1}{2}(\mu+3 p-2 \Lambda) \dot{\xi}_{d} h_{e}^{d}+\frac{1}{2}(\mu-p+2 \Lambda) \dot{\xi}_{d} h_{e}^{d} \\
& =-\frac{1}{2}(\mu+3 p-2 \Lambda) H_{c d} u^{c} h_{e}^{d}+(\mu+p) \dot{\xi}_{d} h_{e}^{d}
\end{aligned}
$$

hence,

$$
\begin{aligned}
\text { LHS }= & -(\mu+p) h_{e d} L_{\xi} u^{d}-\left[\stackrel{\circ}{q}_{d} h_{e}^{d}+q_{c} \xi_{; d}^{c} h_{e}^{d}\right] \\
& +\stackrel{\circ}{\pi}_{d c} u^{d} h_{e}^{c}-h_{e}^{d} \pi_{c d} \dot{\xi}^{c} .
\end{aligned}
$$

The term

$$
\begin{aligned}
\stackrel{\circ}{q}_{d} h_{e}^{d}+q_{c} \xi_{; d}^{c} h_{e}^{d} & =\stackrel{\circ}{q}_{d} h_{e}^{d}+q_{c}\left(\psi \delta_{d}^{c}+H_{d}^{c}-\xi_{d}^{. ; c}\right) h_{e}^{d} \\
& =\left(\stackrel{\circ}{q}_{d}-\xi_{d} ; c q_{c}\right) h_{e}^{d}+\psi q_{e}+H_{c d} q^{c} h_{e}^{d} \\
& =h_{e}^{d} L_{\xi} q_{d}+\psi q_{e}+H_{c d} q^{c} h_{e}^{d} .
\end{aligned}
$$

Also the Lie derivative

$$
L \xi \pi_{c d}=\pi_{c d ; f} \xi^{f}+\pi_{f d} \xi_{; c}^{f}+\pi_{c f} \xi_{; d}^{f}=\stackrel{\AA}{\pi}_{c d}+\pi_{f d} \xi_{; c}^{f}+\pi_{c f} \xi_{; d}^{f} ;
$$

hence,

$$
u^{d} h_{e}^{c} L_{\xi} \pi_{c d}=\stackrel{\circ}{\pi}_{c d} u^{d} h_{e}^{c}+\pi_{c f} \dot{\xi}^{f} h_{e}^{c}
$$

Therefore,

$$
\text { LHS }=-(\mu+p) h_{e d} L_{\xi} u^{d}-h_{e}^{d} L_{\xi} q_{d}+u^{d} h_{e}^{c} L_{\xi} \pi_{c d}-\psi q_{e}-H_{c d} q^{c} h_{e}^{d}
$$

and Eq. (154) becomes

$$
-(\mu+p) h_{e d} L_{\xi} u^{d}-h_{d e} L_{\xi} q^{d}+u^{d} h_{e}^{c} L_{\xi} \pi_{c d}-\psi q_{e}-H_{c d} q^{c} h_{e}^{d}=-\left(-2 q_{\psi e}+q_{K e}\right)
$$

or

$$
-h_{d e} L_{\xi} q^{d}+u^{d} h_{e}^{c} L_{\xi} \pi_{c d}=(\mu+p) h_{e d} L_{\xi} u^{d}+\psi q_{e}+H_{c d} q^{c} h_{e}^{d}-\left(-2 q_{\psi e}+q_{K e}\right) .
$$

We continue with Eq. (156).

We have

$$
\begin{aligned}
& \text { LHS }=2\left[h_{(a}^{e} h_{b)}^{f}-\frac{1}{3} h_{a b} h^{e f}\right] \\
& {\left[\frac{1}{2}(\mu-p)^{\circ} h_{e f}+2 q_{(c} \stackrel{\circ}{d}\right)_{d)} h_{e}^{c} h_{f}^{d}+\stackrel{\circ}{\pi}_{c d} h_{e}^{c} h_{f}^{d}+(\mu-p+2 \Lambda)\left(\psi g_{d r}+H_{d r}\right) h_{e}^{d} h_{f}^{r}+2 q_{r} u_{c} \xi_{; d}^{c} h_{e}^{d} h_{f}^{r}} \\
& \left.\quad+2 \pi_{c r} \xi_{; d}^{c} h_{e}^{d} h_{f}^{r}\right] .
\end{aligned}
$$

The terms

$$
2\left[h_{(a}^{e} h_{b)}^{f}-\frac{1}{3} h_{a b} h^{e f}\right] \frac{1}{2}(\mu-p)^{\circ} h_{e f}=0
$$




$$
\begin{aligned}
& {\left[h_{(a}^{e} h_{b)}^{f}-\frac{1}{3} h_{a b} h^{e f}\right](\mu-p+2 \Lambda) \xi_{d ; r} h_{e}^{d} h_{f}^{r}} \\
& =\left[h_{(a}^{e} h_{b)}^{f}-\frac{1}{3} h_{a b} h^{e f}\right](\mu-p+2 \Lambda)\left(\psi g_{d r}+H_{d r}\right) h_{e}^{d} h_{f}^{r} \\
& =(\mu-p+2 \Lambda)\left[h_{(a}^{e} h_{b)}^{f}-\frac{1}{3} h_{a b} h^{e f}\right] H_{d r} h_{e}^{d} h_{f}^{r} \\
& =(\mu-p+2 \Lambda)\left[h_{(a}^{e} h_{b)}^{f}-\frac{1}{3} h_{a b} h^{e f}\right] H_{e f},
\end{aligned}
$$

i.e., the traceless part of the projection of the traceless tensor $H_{a b}$. The term

$$
\begin{aligned}
& {\left[h_{(a}^{e} h_{b)}^{f}-\frac{1}{3} h_{a b} h^{e f}\right] 2 q_{r} u^{c} \xi_{c ; d} h_{e}^{d} h_{f}^{r}} \\
& =\left[h_{(a}^{e} h_{b)}^{f}-\frac{1}{3} h_{a b} h^{e f}\right] 2 q_{r} u^{c}\left(\psi g_{c d}+H_{c d}\right) h_{e}^{d} h_{f}^{r} \\
& =\left[h_{(a}^{e} h_{b)}^{f}-\frac{1}{3} h_{a b} h^{e f}\right] 2 q_{r} u^{c} H_{c d} h_{e}^{d} h_{f}^{r} \\
& =\left[h_{(a}^{e} h_{b)}^{f}-\frac{1}{3} h_{a b} h^{e f}\right] 2 q_{f} u^{c} H_{c d} h_{e}^{d} \\
& =2 q_{(a} h_{b)}^{d} H_{c d} u^{c}-\frac{2}{3}\left(H_{c d} u^{c} q^{d}\right) h_{a b} .
\end{aligned}
$$

The term

$$
\begin{aligned}
& {\left[h_{(a}^{e} h_{b)}^{f}-\frac{1}{3} h_{a b} h^{e f}\right] 2 \pi_{c r} \xi_{; d}^{c} h_{e}^{d} h_{f}^{r}} \\
& =2\left[h_{(a}^{e} h_{b)}^{f}-\frac{1}{3} h_{a b} h^{e f}\right] \pi_{. r}^{c}\left(\psi g_{c d}+H_{c d}\right) h_{e}^{d} h_{f}^{r} \\
& =2\left[h_{(a}^{e} h_{b)}^{f}-\frac{1}{3} h_{a b} h^{e f}\right]\left(\psi \pi_{e f}+\pi_{. f}^{c} H_{c d} h_{e}^{d}\right) \\
& =2\left[h_{(a}^{e} h_{b)}^{f}-\frac{1}{3} h_{a b} h^{e f}\right] \psi \pi_{e f}+2\left[h_{(a}^{e} h_{b)}^{f}-\frac{1}{3} h_{a b} h^{e f}\right] \pi_{. f}^{c} H_{c d} h_{e}^{d} \\
& =2\left[h_{(a}^{e} h_{b)}^{f}-\frac{1}{3} h_{a b} h^{e f}\right] \pi_{. f}^{c} H_{c d} h_{e}^{d} \\
& =2 h_{(a}^{e} h_{b)}^{f} \pi_{. f}^{c} H_{c d} h_{e}^{d}-\frac{2}{3} h_{a b}\left(\pi^{c d} H_{c d}\right) .
\end{aligned}
$$

Therefore, we have

$$
\begin{aligned}
& 2 q_{(c} \stackrel{\circ}{u}_{d)} h_{e}^{c} h_{f}^{d}+\stackrel{\circ}{\pi}_{c d} h_{e}^{c} h_{f}^{d}+(\mu-p+2 \Lambda)\left[h_{(a}^{e} h_{b)}^{f}-\frac{1}{3} h_{a b} h^{e f}\right] H_{e f} \\
& \quad+2 q_{(a} h_{b)}^{d} H_{c d} u^{c}-\frac{2}{3}\left(H_{c d} u^{c} q^{d}\right) h_{a b} \\
& \quad+2 h_{(a}^{e} h_{b)}^{f} \pi_{. f}^{c} H_{c d} h_{e}^{d}-\frac{2}{3} h_{a b}\left(\pi^{c d} H_{c d}\right) \\
& =\frac{1}{2}\left[\pi_{\psi a b}+\pi_{K a b}\right] .
\end{aligned}
$$

The gravitational field equations are (157), (161), (158) and (162). With these field equations we have completed the scenario for the generic general relativistic model and we pass to the well-known gravitational model Bianchi I model. 


\section{Example: the Bianchi I model}

In the following, we assume the following conventions:

Greek indices take the space values 1,2, 3 and Latin indices the spacetime values $0,1,2,3$. We define the sign of the curvature tensor from the identity $A_{; b c}^{a}-A_{; c b}^{a}=R_{b c d}^{a} A^{d}$ or $A_{a ; b c}-A_{a ; c b}=R_{d a b c} A^{d}$. In terms of the connection coefficients $R_{b c d}^{a}=\Gamma_{d b}^{a}, c-\Gamma_{c b}^{a}, d+\Gamma_{c f}^{a} \Gamma_{d b}^{f}-\Gamma_{d f}^{a} \Gamma_{c b}^{f}$.

\subsection{Geometric assumptions defining the model}

A diagonal Bianchi I spacetime is a spatially homogeneous spacetime which admits an Abelian group of isometries $G_{3}$, acting on spacelike hypersurfaces, generated by the spacelike $\mathrm{KVs} \xi_{1}=\partial_{x}, \xi_{2}=\partial_{y}, \xi_{3}=\partial_{z}$ and a timelike gradient $\mathrm{KV}$ the $u^{a}=\frac{\partial}{\partial t}$ which is normal to the homogeneous 3-D hypersurfaces.

In synchronous coordinates $\{t, x, y, z\}$, the above assumptions imply that the metric of this spacetime is

$$
\mathrm{d} s^{2}=-\mathrm{d} t^{2}+A_{\mu}^{2}(t)\left(\mathrm{d} x^{\mu}\right)^{2},
$$

where the metric functions $A_{1}(t), A_{2}(t), A_{3}(t)$ are functions of the time coordinate only. When two of the functions $A_{\mu}(t)$ are equal (e.g. $A_{2}=A_{3}$ ) the Bianchi I spacetime reduces to the important class of plane symmetric spacetimes (a special class of the Locally Rotational Symmetric spacetimes $[10,27]$ which admit a $G_{4}$ group of isometries acting multiply transitively on the spacelike hypersurfaces of homogeneity generated by the vectors $\xi_{1}, \xi_{2}, \xi_{3}$ and $\xi_{4}=x^{2} \partial_{3}-x^{3} \partial_{2}$ ).

For economy of writing in the following, we write $A_{\mu}$ instead of $A_{\mu}^{2}(t)$. Furthermore, we shall be interested only in proper diagonal Bianchi I spacetimes (which in the following shall be referred simply as Bianchi I spacetimes); hence, all metric functions are assumed to be different and the dimension of the group of isometries acting on the spacelike hypersurfaces is three.

The implications of the additional geometric assumptions (i.e., the symmetries we assumed) are as follows.

1. The computation of the Ricci tensor

$$
\begin{gathered}
R_{t t}=-\frac{\ddot{A}_{1} A_{2} A_{3}+\ddot{A}_{2} A_{1} A_{3}+\ddot{A}_{3} A_{1} A_{2}}{A_{1} A_{2} A_{3}}, \\
R_{x x}=\frac{A_{1}\left(\ddot{A}_{1} A_{2} A_{3}+\dot{A}_{1} \dot{A}_{2} A_{3}+\dot{A}_{1} \dot{A}_{3} A_{2}\right)}{A_{2} A_{3}}, \\
R_{y y}=\frac{A_{2}\left(\ddot{A}_{2} A_{1} A_{3}+\dot{A}_{1} \dot{A}_{2} A_{3}+\dot{A}_{2} \dot{A}_{3} A_{1}\right)}{A_{1} A_{3}}, \\
R_{z z}=\frac{A_{3}\left(\ddot{A}_{3} A_{1} A_{2}+\dot{A}_{1} \dot{A}_{3} A_{2}+\dot{A}_{2} \dot{A}_{3} A_{1}\right)}{A_{1} A_{2}} .
\end{gathered}
$$

From the Ricci tensor, we compute the Einstein tensor and then use Einstein field equations to write the energy-momentum tensor in terms of the metric functions $A_{1}(t), A_{2}(t), A_{3}(t)$ and their derivatives. However, that does not mean that we are able to discuss anything about the physical variables (energy density, isotropic pressure, etc.) because to do that we need to have observers. The expression of $T_{a b}$ we find for $T_{a b}$ is the matter content of this spacetime, the same for all observers in the Bianchi I background.

2. The computation of the Weyl tensor

The Weyl tensor defined in (45) is important because it is involved in the second Bianchi identity. More specifically, from this tensor one computes the electric and the magnetic parts $E^{a b}, H^{a b}$ which enter into the $1+3$ decomposition of the second Bianchi identity given by Eqs. (48)-(51). We compute 


$$
\begin{aligned}
& E_{x x}=\frac{A_{1}\left(-2 \ddot{A}_{1} A_{2} A_{3}+\dot{A}_{1} \dot{A}_{2} A_{3}+\dot{A}_{1} \dot{A}_{3} A_{2}+\ddot{A}_{2} A_{1} A_{3}+\ddot{A}_{3} A_{1} A_{2}-2 \dot{A}_{2} \dot{A}_{3} A_{1}\right)}{6 A_{2} A_{3}} . \\
& E_{y y}=\frac{A_{2}\left(-2 \ddot{A}_{2} A_{1} A_{3}+\dot{A}_{1} \dot{A}_{2} A_{3}+\dot{A}_{2} \dot{A}_{3} A_{1}+\ddot{A}_{1} A_{2} A_{3}+\ddot{A}_{3} A_{1} A_{2}-2 \dot{A}_{1} \dot{A}_{3} A_{2}\right)}{6 A_{1} A_{3}} . \\
& E_{z z}=-\frac{A_{3}\left(2 \ddot{A}_{3} A_{1} A_{2}-\dot{A}_{1} \dot{A}_{3} A_{2}-\dot{A}_{2} \dot{A}_{3} A_{1}-\ddot{A}_{1} A_{2} A_{3}-\ddot{A}_{2} A_{1} A_{3}+2 \dot{A}_{1} \dot{A}_{2} A_{3}\right)}{6 A_{1} A_{2}}
\end{aligned}
$$

and the magnetic part $H_{a b}=0$.

Observers

The choice of observers is open and independent of the choice of the assumed symmetries, (i.e., the model) spacetime. However, as it has been noted, the kinematic quantities they define must satisfy the propagation, the constraint equations and the Bianchi second identity.

We choose the observers (this is one choice, any other would do provided it satisfies the aforementioned identities) to be the ones defined by the time coordinate $t$, i.e., we take $u^{a}=\delta_{0}^{a}$ in the synchronous coordinate system.

The implications of this choice of observers are

1. Kinematics

We $1+3$ decompose $u_{a ; b}$ and find the kinematic variables:

$$
\begin{aligned}
\theta & =\left[\ln \left(A_{1} A_{2} A_{3}\right)\right]^{\circ} \\
\omega_{a b} & =0, \dot{u}^{a}=0 \\
\sigma_{a b} & =\frac{1}{3} \operatorname{diag}\left(0, A_{1}^{2}\left[\ln \left(\frac{A_{1}^{2}}{A_{2} A_{3}}\right)\right], A_{2}^{2}\left[\ln \left(\frac{A_{2}^{2}}{A_{3} A_{1}}\right)\right], A_{3}^{2}\left[\ln \left(\frac{A_{3}^{2}}{A_{1} A_{2}}\right)\right]\right) .
\end{aligned}
$$

a. The propagation equations give ${ }^{17}$ :

$$
\begin{aligned}
& \dot{\theta}+\frac{1}{3} \theta^{2}+2 \sigma^{2}=\frac{\ddot{A}_{1} A_{2} A_{3}+\ddot{A}_{2} A_{1} A_{3}+\ddot{A}_{3} A_{1} A_{2}}{A_{1} A_{2} A_{3}}, \\
& -E_{s t}=h_{s}^{a} h_{t}^{b} \dot{\sigma}_{a b}+\sigma_{s c} \sigma_{t}^{c}+\frac{2}{3} \sigma_{s t} \theta-\frac{2}{3} 2 \sigma^{2} h_{s t} .
\end{aligned}
$$

b. The constraint equations give:

$$
\begin{aligned}
& \frac{2}{3} h_{s}^{c} \theta,,_{c}=h_{s}^{c} h^{a b} \sigma_{a c ; b} \text { (three equations), } \\
& 0=-h_{(s}^{a} h_{t)}^{b} \sigma_{b}^{c ; d} \eta_{a r c d} u^{r} \text { (five equations). }
\end{aligned}
$$

Equations (174)-(177) must be satisfied identically by the kinematic quantities. It is easy to show that this is true for Eqs. (174) and (175). Equation (176) is trivially satisfied because $\theta, \sigma_{a b}$ are functions of $t$ only. Equation (177) is also trivially satisfied because it contains derivatives of the components of $\sigma_{a b}$ along the space coordinates only (due to the term $\eta_{\operatorname{arcd}} u^{r}$ ). We conclude that the propagation and the constraint equations do not give any new conditions on the metric functions $A_{1}(t), A_{2}(t), A_{3}(t)$.

c. The Bianchi identities give the derivatives of $E_{s t}, H_{s t}$; therefore, they do not add new constraints on the metric functions. They are only compatibility conditions.

${ }^{17}$ Note that $\sigma^{2}=\frac{1}{2} \sigma_{a b} \sigma^{a b}$. For the case, we are considering we calculate:

$$
\sigma^{2}=\frac{1}{3}\left[\sum_{I=1}^{3}\left(\frac{\dot{A}_{I}}{A_{I}}\right)^{2}-\sum_{I \neq J=1}^{3} \frac{\dot{A}_{I}}{A_{I}} \frac{\dot{A}_{J}}{A_{J}}\right]
$$


d. The propagation of the kinematic quantities along the symmetry vectors.

The fact that $\xi_{\mu}^{a}(\mu=1,2,3)$ are Killing vectors (hence $\left.\psi=0, H_{a b}=0\right)$ provides:

$$
V^{a}(u)=\hat{V}_{a}(u)=0 .
$$

From (115) and (117), we have taking into account the above results:

$$
\begin{gathered}
L_{\xi} \theta=0 \Rightarrow \theta=\theta(t), \\
L_{\xi} \sigma_{a b}=0 \Rightarrow \sigma_{a b}=\sigma_{a b}(t) .
\end{gathered}
$$

These equations give nothing new because we have already computed $\theta, \sigma_{a b}$ and have found that they are functions of $t$ only.

Dynamics

a. We compute the physical parameters for the chosen observers. We find:

$$
\begin{aligned}
\mu & =\frac{\dot{A}_{1} \dot{A}_{2} A_{3}+\dot{A}_{1} \dot{A}_{3} A_{2}+\dot{A}_{2} \dot{A}_{3} A_{1}}{A_{1} A_{2} A_{3}}=\frac{\dot{A}_{1} \dot{A}_{2}}{A_{1} A_{2}}+\frac{\dot{A}_{1} \dot{A}_{3}}{A_{1} A_{3}}+\frac{\dot{A}_{2} \dot{A}_{3}}{A_{2} A_{3}}, \\
p & =-\frac{2 \ddot{A}_{2} A_{1} A_{3}+2 \ddot{A}_{3} A_{1} A_{2}+\dot{A}_{2} \dot{A}_{3} A_{1}+2 \ddot{A}_{1} A_{2} A_{3}+\dot{A}_{1} \dot{A}_{3} A_{2}+\dot{A}_{1} \dot{A}_{2} A_{3}}{3 A_{1} A_{2} A_{3}}, \\
\pi_{x x} & =-\frac{A_{1}\left(-2 \ddot{A}_{1} A_{2} A_{3}+\ddot{A}_{2} A_{1} A_{3}+\ddot{A}_{3} A_{1} A_{2}+2 \dot{A}_{2} \dot{A}_{3} A_{1}-\dot{A}_{3} \dot{A}_{1} A_{2}-\dot{A}_{1} \dot{A}_{2} A_{3}\right)}{3 A_{2} A_{3}}, \\
\pi_{y y} & =-\frac{A_{2}\left(-2 \ddot{A}_{2} A_{1} A_{3}+\ddot{A}_{3} A_{1} A_{2}+\ddot{A}_{1} A_{2} A_{3}+2 \dot{A}_{1} \dot{A}_{3} A_{2}-\dot{A}_{2} \dot{A}_{3} A_{1}-\dot{A}_{1} \dot{A}_{2} A_{3}\right)}{3 A_{1} A_{3}}, \\
\pi_{z z} & =-\frac{A_{3}\left(-2 \ddot{A}_{3} A_{1} A_{2}+\ddot{A}_{2} A_{1} A_{3}+\ddot{A}_{1} A_{2} A_{3}+2 \dot{A}_{1} \dot{A}_{2} A_{3}-\dot{A}_{2} \dot{A}_{3} A_{1}-\dot{A}_{1} \dot{A}_{3} A_{2}\right)}{3 A_{1} A_{2}} .
\end{aligned}
$$

The momentum transfer vector $q^{a}=0 .{ }^{18}$

We note that the second equation can be written:

$$
\frac{\ddot{A}_{1}}{A_{1}}+\frac{\ddot{A}_{2}}{A_{2}}+\frac{\ddot{A}_{3}}{A_{3}}=-\frac{1}{2}(\mu+3 p) .
$$

We introduce the notation:

$$
\begin{aligned}
& I_{2}=\frac{\ddot{A}_{1}}{A_{1}}+\frac{\ddot{A}_{2}}{A_{2}}+\frac{\ddot{A}_{3}}{A_{3}}=-\frac{1}{2}(\mu+3 p) \\
& I_{1}=\frac{\dot{A}_{1} \dot{A}_{2}}{A_{1} A_{2}}+\frac{\dot{A}_{1} \dot{A}_{3}}{A_{1} A_{3}}+\frac{\dot{A}_{2} \dot{A}_{3}}{A_{2} A_{3}}=\mu
\end{aligned}
$$

and we have

$$
\begin{aligned}
\mu & =I_{1}, \\
p & =-\frac{1}{3}\left(2 I_{2}+I_{1}\right) \\
\pi_{x x} & =\frac{A_{1}^{2}}{3}\left(3 \frac{\ddot{A}_{1}}{A_{1}}-3 \frac{\dot{A}_{2} \dot{A}_{3}}{A_{2} A_{3}}-I_{2}+I_{1}\right), \\
\pi_{y y} & =\frac{A_{2}^{2}}{3}\left(3 \frac{\ddot{A}_{2}}{A_{2}}-3 \frac{\dot{A}_{1} \dot{A}_{3}}{A_{1} A_{3}}-I_{2}+I_{1}\right),
\end{aligned}
$$

\footnotetext{
18 This is expected from the symmetries of the metric and the non-degeneracy of the $T_{a b}$. Note that both $R_{a b}$ and $T_{a b}$ are of the same form as the metric. This is to be expected because they can be considered as metrics (a metric is a symmetric tensor of type $(0,2)$ and nothing more or less) and they admit the same KVs with the metric.
} 


$$
\pi_{z z}=\frac{A_{3}^{2}}{3}\left(3 \frac{\ddot{A}_{3}}{A_{3}}-3 \frac{\dot{A}_{1} \dot{A}_{2}}{A_{1} A_{2}}-I_{2}+I_{1}\right)
$$

b. Conservation equations.

From (129) and (130) we have, for Bianchi I spacetime and the observers we selected the conservation equations:

$$
\begin{aligned}
& \dot{\mu}+(\mu+p) \theta+\pi^{a b} \sigma_{a b}=0 \\
& h_{a}^{c}\left(p_{, c}+\pi_{c ; d}^{d}\right)=0 .
\end{aligned}
$$

Equation (191) is trivially satisfied because all quantities are functions of $t$. Equation (190) is also trivially satisfied if we replace the expressions of $\mu, p, \theta, \pi_{a b}$ from the corresponding expressions.

We see that there are no field equations to solve! Indeed we have solved them in terms of three arbitrary functions which are the metric functions $A_{1}(t), A_{2}(t), A_{3}(t)$ ! Therefore, for the observers $u^{a}=\delta_{0}^{a}$ we have solved the problem completely. ${ }^{19}$

We are free to select special solutions from the three parameter family of solutions we have found by imposing extra additional requirements. In the following section, we make one such requirement and consider those Bianchi I spacetime which for the observers we have chosen give rise to a special type of mater which we call string fluid. Needless to say that one could consider other requirements and select other types of matter for the same spacetime and the same observers. Every specification/condition on the metric functions $A_{1}(t), A_{2}(t), A_{3}(t)$ will produce a model Bianchi I spacetime (physical or not).

\section{The string fluid}

The connection between strings and vortices is well known [15,16,19,23]. In particular, a geometric or Nambu string is a two-dimensional timelike surface in spacetime. Letelier [17] has considered a fluid represented by a combination of geometric strings with particles attached to them so that both have the same four velocity. He called such a fluid a string fluid and he studied the gravitational field it produces in given spacetime backgrounds. In a series of papers, various authors [2-4,25,30,33,34] [31] have considered various types of collineations for a string fluid and derived the conditions which must be satisfied for the string fluid to admit a given collineation.

In this work, we consider the string fluid with particles attached to the strings which for some observers $u^{a}$ is described by the energy-momentum tensor ${ }^{20}[15,16]$ :

$$
T_{a b}=\rho\left(u_{a} u_{b}-n_{a} n_{b}\right)+q p_{a b},
$$

where $\rho=\rho_{p}+\rho_{s}$ is the sum of the mass density of the strings $\left(\rho_{s}\right)$ and the mass density of the particles $\left(\rho_{p}\right)$, $u^{a}$ is the common four velocity $\left(u^{a} u_{a}=-1\right)$ of the string and the attached particle, $n^{a}$ is a unit spacelike vector $\left(n^{a} n_{a}=1\right)$ normal to $u^{a}\left(u^{a} n_{a}=0\right)$, which specifies the direction of the string (and the direction of anisotropy of the string fluid), $q$ is a parameter contributing to the dynamic and kinematic properties of the string, $p_{a b}=h_{a b}-n_{a} n_{b}$ is the screen projection operator defined by the vectors $u^{a}, n^{a}$.

By rewriting the energy momentum tensor as

$$
T_{a b}=\rho u_{a} u_{b}+\frac{1}{3}(2 q-\rho) h_{a b}+(q+\rho)\left(\frac{1}{3} h_{a b}-n_{a} n_{b}\right)
$$

(or otherwise) we compute its $1+3$ decomposition. It follows that for a string fluid:

$$
\mu=\rho, \quad p=\frac{1}{3}(2 q-\rho), \quad q^{a}=0, \quad \pi_{a b}=(q+\rho)\left(\frac{1}{3} h_{a b}-n_{a} n_{b}\right) .
$$

We conclude that a string fluid is an anisotropic fluid with vanishing heat flux. Furthermore, we note that $n^{a}$ is an eigenvector of the anisotropic stress tensor $\pi_{a b}$ with eigenvalue $-\frac{2}{3}(q+\rho)$. We assume $q+\rho \neq 0$

19 For another class of observers we could have more constraint equations which would have to be solved. Obviously in this case all dynamic and kinematic variables will (in general) be different.

${ }^{20}$ This expression is found from Eq. (2.28a) of [15] if we set $\rho=-\sigma$ and $\pi \rightarrow-\pi$. 
otherwise the string fluid reduces to a perfect fluid with energy momentum tensor $T_{a b}=q g_{a b}$. This fluid has the unphysical equation of state $\mu+p=0$.

From the above, we note that the structure of the energy momentum tensor is compatible with the general expressions (179)-(183); therefore, the model of a string fluid we considered is possible to be described in a Bianchi I spacetime background with the comoving observers $u^{a}=\delta_{0}^{a}$ we considered in the previous section. This is equivalent to say that the additional symmetry assumptions we did is compatible in the Bianchi I model spacetime with the form of the energy-momentum tensor (192). This does not mean that there are not different sets of additional assumptions which are compatible with matter of the form (192). Bianchi I is just one.

To find the specific metric functions $A_{1}(t), A_{2}(t), A_{3}(t)$ which correspond (or select) the string fluid model we equate the dynamical parameters of (179)-(183) with those of (194). We assume the string direction to be along the $x$-axis, i.e., we take $\left(A_{1} \neq 0\right)$ :

$$
n^{a}=\frac{1}{\sqrt{A_{1}}} \delta_{1}^{a}
$$

From the mass density and the pressure, we find:

$$
\begin{gathered}
\mu+3 p=2 q \Rightarrow I_{2}=-q \\
\pi_{a b}=(q+\rho)\left(\frac{1}{3} h_{a b}-A_{1}^{2} \delta_{a}^{1} \delta_{b}^{1}\right)=\left(\rho-I_{2}\right)\left(\frac{1}{3} h_{a b}-A_{1}^{2} \delta_{a}^{1} \delta_{b}^{1}\right) .
\end{gathered}
$$

In the coordinates we use:

$$
h_{a b}=\operatorname{diag}\left(0, A_{1}^{2}, A_{2}^{2} \cdot A_{3}^{2}\right),
$$

replacing in the expression of $\pi_{a b}$, we find:

$$
\pi_{a b}=\frac{1}{3}\left(\rho-I_{2}\right) \operatorname{diag}\left(0,-2 A_{1}^{2}, A_{2}^{2}, A_{3}^{2}\right) .
$$

Equating the two expressions of $\pi_{a b}$ (187) - (189) and (198), we find the field equations:

$$
\begin{aligned}
& \frac{\ddot{A}_{1}}{A_{1}}-\frac{\dot{A}_{2} \dot{A}_{3}}{A_{2} A_{3}}=-\rho+I_{2}=-(\rho+q) \\
& \frac{\ddot{A_{2}}}{A_{2}}-\frac{\dot{A}_{1} \dot{A}_{3}}{A_{1} A_{3}}=0 \\
& \frac{\ddot{A}_{3}}{A_{3}}-\frac{\dot{A}_{1} \dot{A}_{2}}{A_{1} A_{2}}=0 .
\end{aligned}
$$

The last three equations are dependent (one follows from the other two). Eventually, we have the following system of four simultaneous equations for the five unknowns: $A_{1}(t), A_{2}(t), A_{3}(t), q, \rho$ :

$$
\begin{aligned}
& \frac{\dot{A}_{1} \dot{A}_{2}}{A_{1} A_{2}}+\frac{\dot{A}_{1} \dot{A}_{3}}{A_{1} A_{3}}+\frac{\dot{A}_{2} \dot{A}_{3}}{A_{2} A_{3}}=\rho, \\
& \frac{\ddot{A}_{1}}{A_{1}}+\frac{\ddot{A}_{2}}{A_{2}}+\frac{\ddot{A}_{3}}{A_{3}}=-q, \\
& \frac{\ddot{A_{2}}}{A_{2}}-\frac{\dot{A}_{1} \dot{A}_{3}}{A_{1} A_{3}}=0, \\
& \frac{\ddot{A_{3}}}{A_{3}}-\frac{\dot{A}_{1} \dot{A}_{2}}{A_{1} A_{2}}=0 .
\end{aligned}
$$

We have still the freedom to specify one more condition. This condition could be an equation of state (in the broad sense). 
Note that the kinematic variables are not effected. Therefore, when we take the equation of state and we determine the functions $A_{1}(t), A_{2}(t), A_{3}(t)$, we can compute the kinematic variables $\theta, \sigma_{a b}$ and draw conclusions on the kinematics of the string fluid.

\subsection{An alternative energy-momentum tensor}

Instead of the energy-momentum tensor (192), Lattelier [17] considered the energy-momentum tensor

$$
T_{a b}=\rho u_{a} u_{b}-\lambda n_{a} n_{b} .
$$

This tensor reduces to the one we have considered if we set $q=0$ and $\rho=\lambda$. In general, it is different to the one we have discussed above. However, our analysis applies the same. Considering the same observers and the same direction $n^{a}$ we have the following $1+3$ decomposition of $T_{a b}$ :

$$
T_{a b}=\rho u_{a} u_{b}-\frac{1}{3} \lambda h_{a b}+\lambda\left(\frac{1}{3} h_{a b}-n_{a} n_{b}\right)
$$

from which follows:

$$
\begin{aligned}
\mu & =\rho, p=-\frac{1}{3} \lambda, q_{a}=0 \\
\pi_{a b} & =\lambda\left(\frac{1}{3} h_{a b}-n_{a} n_{b}\right)=\frac{\lambda}{3}\left(0,-2 A_{1}^{2}, A_{2}^{2}, A_{3}^{2}\right) .
\end{aligned}
$$

Then

$$
I_{2}=-\frac{1}{2}(\mu+3 p)=-\frac{1}{2}(\rho-\lambda) .
$$

Equating the two expressions of $\pi_{a b}$ (187)-(189) and (206), we find the field equations:

$$
\begin{aligned}
& \frac{\ddot{A}_{1}}{A_{1}}-\frac{\dot{A}_{2} \dot{A}_{3}}{A_{2} A_{3}}=-\lambda+I_{2}=-\frac{1}{6}(5 \lambda+3 \rho) \\
& \frac{\ddot{A}}{A_{2}}-\frac{\dot{A}_{1} \dot{A}_{3}}{A_{1} A_{3}}=I_{2}=\frac{1}{2}\left(\frac{\lambda}{3}-\rho\right) \\
& \frac{\ddot{A}_{3}}{A_{3}}-\frac{\dot{A}_{1} \dot{A}_{2}}{A_{1} A_{2}}=\frac{\ddot{A}_{2}}{A_{2}}-\frac{\dot{A}_{1} \dot{A}_{3}}{A_{1} A_{3}} .
\end{aligned}
$$

The last three equations are dependent (one follows from the other two). Eventually, we have again the following system of four simultaneous equations for the five unknowns: $A_{1}(t), A_{2}(t), A_{3}(t), \lambda(t), \rho(t)$ :

$$
\begin{aligned}
& \frac{\dot{A}_{1} \dot{A}_{2}}{A_{1} A_{2}}+\frac{\dot{A}_{1} \dot{A}_{3}}{A_{1} A_{3}}+\frac{\dot{A}_{2} \dot{A}_{3}}{A_{2} A_{3}}=\rho, \\
& \frac{\ddot{A_{1}}}{A_{1}}+\frac{\ddot{A}_{2}}{A_{2}}+\frac{\ddot{A}_{3}}{A_{3}}=\frac{1}{2}(\lambda-\rho), \\
& \frac{\ddot{A_{2}}}{A_{2}}-\frac{\dot{A}_{1} \dot{A}_{3}}{A_{1} A_{3}}=\frac{1}{2}(\lambda-\rho), \\
& \frac{\ddot{A_{3}}}{A_{3}}-\frac{\dot{A}_{1} \dot{A}_{2}}{A_{1} A_{2}}=\frac{1}{2}(\lambda-\rho) .
\end{aligned}
$$

We note that in this choice one has again the freedom to consider an extra condition/equation of state. The solution of the field equations will be the starting point of making Physics with this model. 


\section{The $1+1+2$ decomposition wrt a double congruence}

It is possible in a gravitational model one has in addition to the four-velocity $u^{a}$ an additional non-null vector field $n^{a}$ which is not parallel to $u^{a}$, as it is the case, for example, with the string fluid. The existence of two characteristic vector fields in spacetime introduces the concept of the double congruence which leads to the finer $1+1+2$ decomposition of tensor fields in spacetime. This decomposition introduces new "kinematic" variables for the vector field $n^{a}$ and new dynamical fields from the $1+1+2$ decomposition of the energy momentum tensor $T_{a b}$.

Consider two unit vector fields $u^{i}$ and $n^{i}$, with signatures $u^{i} u_{i}=\varepsilon(u), n^{i} n_{i}=\varepsilon(n)$ so that $u^{i} n_{i}=\phi$, where $\phi \neq \pm 1$ (i.e., $u^{i}$ and $n^{i}$ are not parallel) and define the screen projection tensor $P_{i j}(u, n)$ by the formula:

$$
P_{i j}(u, n)=g_{i j}+\frac{1}{\Delta}\left[\varepsilon(n) u_{i} u_{j}+\varepsilon(u) n_{i} n_{j}-\phi\left(u_{i} n_{j}+n_{i} u_{j}\right)\right],
$$

where $\Delta=\phi^{2}-\varepsilon(u) \varepsilon(n)$.

It is easy to show the properties:

$$
\begin{gathered}
P_{i j}=P_{j i}, P_{i j} P_{k}^{j}=P_{i k}, P_{i}^{i}=2 \\
\quad\left(P_{i j} \text { is a symmetric metric in the 2-space spanned by }\left(u^{i}, n^{i}\right)\right) \\
P_{i j} u^{i}=P_{i j} n^{i}=0 \\
\quad\left(P_{i j} \text { projects normal to both }\left(u^{i}, n^{i}\right) \text { that is the "screen space" spanned }{ }^{21} \text { by }\left(u^{i}, n^{i}\right) .\right.
\end{gathered}
$$

When the vector fields $u^{i}, n^{i}$ are timelike $(\varepsilon(u)=-1)$ and spacelike $(\varepsilon(n)=+1)$, respectively, the above formula becomes

$$
P_{i j}(u, n)=g_{i j}-\frac{1}{1+\phi^{2}}\left[-u_{i} u_{j}+n_{i} n_{j}+\phi\left(u_{i} n_{j}+n_{i} u_{j}\right)\right] .
$$

Furthermore, when $u^{i}, n^{i}$ are perpendicular, then $\phi=0$, and the formula for $P_{i j}(u, n)$ reduces further to

$$
P_{i j}(u, n)=g_{i j}+u_{i} u_{j}-n_{i} n_{j}=h_{i j}-n_{i} n_{j},
$$

where $h_{i j}=g_{i j}+u_{i} u_{j}$ is the projection tensor for $u^{i}$. The two unit vector fields $\left\{u^{i}, n^{i}\right\}$ define a double congruence which with the use of the projection tensor $P_{i j}(u, n)$ define the $1+1+2$ decomposition of geometric objects in spacetime. We start with the following.

Proposition 15.1 A vector field (null or not) $R^{i}$ is $1+1+2$ decomposed wrt the double congruence $\left\{u^{i}, n^{i}\right\}$ by means of the following identity:

$$
R^{i}=-\frac{1}{\Delta}\left[\left(\varepsilon(n) R_{u}-\phi R_{n}\right) u^{i}+\left(\varepsilon(u) R_{n}-\phi R_{u}\right) n^{i}\right]+P_{j}^{i} R^{j},
$$

where $R_{u}=R^{i} u_{i}$ and $R_{n}=R^{i} n_{i}$.

Proof Assume that

$$
R^{i}=A u^{i}+B n^{i}+P_{j}^{i} R^{j}
$$

Contract with $u^{i}, n^{i}$ to get the system of equations:

$$
\begin{aligned}
\varepsilon(u) A+\phi B & =R_{u}, \\
\phi A+\varepsilon(n) B & =R_{n},
\end{aligned}
$$

where $R_{u}=R^{i} u_{i}$ and $R_{n}=R^{i} n_{i}$. The determinant of the system is $\varepsilon(u) \varepsilon(n)-\phi^{2}=-\Delta$. The solution is

$$
\begin{aligned}
A & =-\frac{1}{\Delta}\left(\varepsilon(n) R_{u}-\phi R_{n}\right) \\
B & =-\frac{1}{\Delta}\left(\varepsilon(u) R_{n}-\phi R_{u}\right) .
\end{aligned}
$$


Proposition 15.2 The second rank tensor field $Y_{i j}$ is $1+1+2$ decomposed wrt the double congruence $\left\{u^{i}, n^{i}\right\}$ by means of the identity:

$$
Y_{i j}=\frac{1}{\Delta}\left[\alpha u_{i} u_{j}+\beta n_{i} n_{j}+\gamma u_{i} n_{j}+\delta n_{i} u_{j}-\theta_{k} P_{i}^{k} u_{j}+\kappa_{k} P_{i}^{k} n_{j}-\rho_{s} P_{j}^{s} u_{i}+v_{k} n_{i} P_{j}^{k}\right]+P_{i}^{k} P_{j}^{r} Y_{k r},
$$

where

$$
\begin{aligned}
\alpha & =\frac{1}{\Delta}\left[Y_{u u}-\phi \varepsilon(n) Y_{u n}-\phi \varepsilon(n) Y_{n u}+\phi^{2} Y_{n n}\right] \\
\beta & =\frac{1}{\Delta}\left[Y_{n n}-\phi \varepsilon(u) Y_{n u}-\phi \varepsilon(u) Y_{u n}+\phi^{2} Y_{u u}\right] \\
\gamma & =\frac{1}{\Delta}\left[\left(\varepsilon(n) \varepsilon(u) Y_{u n}-\phi \varepsilon(n)\right) Y_{u u}-\phi \varepsilon(u) Y_{n n}+\phi^{2} Y_{n u}\right] \\
\delta & =\frac{1}{\Delta}\left[\varepsilon(n) \varepsilon(u) Y_{n u}-\phi \varepsilon(u) Y_{n n}-\phi \varepsilon(n) Y_{u u}+\phi^{2} Y_{u n}\right] \\
\theta_{k} & =\left[\varepsilon(n) Y_{k r} u^{r}-\phi n^{s} Y_{r s}\right] \\
\kappa_{r} & =\left[-\varepsilon(u) Y_{r s} n^{s}+\phi u^{s} Y_{r s}\right] \\
\rho_{s} & =\left[\varepsilon(n) Y_{r s} u^{r}-\phi Y_{r s} n^{r}\right] \\
v_{r} & =\left[-\varepsilon(u) Y_{r s} n^{r}+\phi Y_{r s} u^{r}\right] \\
\Delta & =\phi^{2}-\varepsilon(s) \varepsilon(n) .
\end{aligned}
$$

Corollary 15.3 Let $u^{i}$ be a unit timelike vector $(\varepsilon(u)=-1)$ and $n^{i}$ a unit spacelike vector $(\varepsilon(n)=1)$ normal to $u^{i}$, that is, $u^{i} n_{i}=\phi=0$. Then Eqs. (211), (214) and (215) become

$$
\begin{aligned}
P_{i j}(u, n)= & g_{i j}+u_{i} u_{j}-n_{i} n_{j}, \\
R^{i}= & -\left(R^{j} u_{j}\right) u^{i}+\left(R^{j} n_{j}\right) n^{i}+R^{j} P_{j}^{i}, \\
Y_{i j}= & \left(Y_{k r} u^{k} u^{r}\right) u_{i} u_{j}+\left(Y_{k r} n^{k} n^{r}\right) n_{i} n_{j}, \\
& -\left(Y_{k r} u^{k} n^{r}\right) u_{i} n_{j}-\left(Y_{k r} n^{k} u^{r}\right) n_{i} u_{j} \\
& -\left(Y_{s r} u^{s}\right) P_{i}^{r} u_{j}-\left(Y_{s r} u^{r}\right) P_{j}^{s} u_{i}+\left(Y_{s r} n^{s}\right) P_{i}^{r} n_{j}+\left(Y_{s r} n^{r}\right) P_{j}^{s} n_{i}+Y_{k r} P_{i}^{k} P_{j}^{r} .
\end{aligned}
$$

In matrix form, the decomposition of $Y_{i j}$ is written as follows ${ }^{22}$ :

$$
Y_{i j} \rightarrow\left(\begin{array}{lll}
\left(Y_{k r} u^{k} u^{r}\right) & -\left(Y_{k r} u^{k} n^{r}\right) & -\left(Y_{s r} u^{r} P_{i}^{r}\right) \\
-\left(Y_{k r} n^{k} u^{r}\right) & \left(Y_{k r} n^{k} n^{r}\right) & \left(Y_{s r} n^{r}\right) P_{j}^{s} n_{i} \\
-\left(Y_{s r} u^{r}\right) P_{j}^{s} u_{i} & \left(Y_{s r} n^{s}\right) P_{i}^{r} n_{j} & Y_{k r} P_{i}^{k} P_{j}^{r}
\end{array}\right) .
$$

By means of this decomposition we brake $Y_{i j}$ in irreducible parts which is easier to study (because they are simpler).

\subsection{Applications}

We apply the previous general decomposition of an arbitrary second rank tensor to various cases which interest the physical applications. In the following, we assume that the pair of vectors defining the $1+1+2$ decomposition are the vectors $u^{a}, n^{a}$ which are timelike $(\varepsilon(u)=-1)$ and spacelike $\left(n^{a}=1\right)$, respectively, and in addition they are normal to each other so that $\phi=0$. Under these assumptions, the decomposition of the symmetric tensor becomes (218) or (219) and the coefficients of Eq. (215) read:

$$
A_{00}=Y_{11}=Y_{k r} u^{k} u^{r}
$$

\footnotetext{
${ }^{22}$ The matrix form of the decomposition is useful in the computation of the various irreducible parts by means of algebraic computing programs.
} 


$$
\begin{aligned}
A_{01} & =-Y_{12}=-Y_{k r} u^{k} n^{r} \\
A_{10} & =-Y_{21}=-Y_{k r} n^{k} u^{r} \\
A_{11} & =Y_{22}=Y_{k r} n^{k} n^{r} \\
\widetilde{A_{i}} & =-Y_{1 t} P_{i}^{t}=-Y_{s r} u^{s} P_{i}^{r} \\
\widetilde{B_{i}} & =Y_{2 t} P_{i}^{t}=Y_{s r} n^{s} P_{i}^{r} \\
\widetilde{C}_{i} & =-Y_{t 1} P_{i}^{t}=-Y_{s r} u^{r} P_{i}^{r} \\
\widetilde{D_{i}} & =Y_{t 2} P_{i}^{t}=Y_{s r} n^{r} P_{j}^{s} \\
\widetilde{C_{i j}} & =P_{[i}^{k} P_{j]}^{r} Y_{k ; r} \\
\widetilde{D_{i j}} & =\left[P_{(i}^{k} P_{j)}^{r}-\frac{1}{2} P_{i j} P^{k r}\right] Y_{k ; r} \\
\widetilde{E} & =P^{k r} Y_{k ; r}
\end{aligned}
$$

\subsubsection{The $1+1+2$ decomposition of the tensor $u_{a ; b}$}

We find the $1+1+2$ decomposition of the derivative $u_{i ; j}$, where $u_{i}$ is the timelike unit vector defining the decomposition. We compute:

$$
\begin{aligned}
& A_{00}: u_{i ; j} u^{i} u^{j}=0 \\
& A_{01}: u_{i ; j} u^{i} n^{b}=0 \\
& A_{10}: u_{i ; j} n^{i} u^{j}=\dot{u}^{i} n_{i} \\
& A_{11}: u_{i ; j} n^{i} n^{j}=*^{*} n_{i} \\
& A_{i}: P_{i}^{j} u_{j ; k} u^{k}=P_{i}^{j} \dot{u}_{j} \\
& \widetilde{A}_{i}:-u_{j ; k} u^{j} P_{i}^{k}=0 \\
& \widetilde{B}_{i}: u_{i ; k} n^{i} P_{j}^{k} n^{i}=P_{i}^{k}\left(\omega_{j k} n^{j}+\sigma_{j k} n^{j}\right)=P_{i}^{k}\left(-\omega_{k r} n^{r}+\sigma_{k r} n^{r}\right) \\
& \widetilde{C}_{i}:-u_{j ; k} u^{k} P_{i}^{j}=-P_{i}^{j} \dot{u}_{j} \\
& \widetilde{D}_{i}: u_{j ; k} n^{k} P_{i}^{j}=P_{i}^{j} u_{j} \\
& \mathcal{R}_{i j}: P_{[i}^{k} P_{j]}^{r} u_{k ; r}=P_{[i}^{k} P_{j]}^{r} \omega_{k r} \\
& \mathcal{S}_{i j}:\left[P_{(i}^{k} P_{j)}^{r}-\frac{1}{2} P_{i j} P^{k r}\right] u_{k ; r}=\left[P_{(i}^{k} P_{j)}^{r}-\frac{1}{2} P_{i j} P^{k r}\right] \sigma_{k r} \\
& \mathcal{E}: P^{k r} u_{k ; r}=-\sigma_{k r} n^{k} n^{r}+\frac{2}{3} \theta .
\end{aligned}
$$

Therefore, the $1+1+2$ decomposition of $u_{i ; j}$ is expressed as

$$
\begin{aligned}
u_{i ; j}= & -\left(\dot{u}^{k} n_{k}\right) n_{i} u_{j}+\left(\sigma_{r k} n^{r} n^{k}+\frac{1}{3} \theta\right) n_{i} n_{j}-P_{i}^{k} \dot{u}_{k} u_{j}+P_{i}^{k} \stackrel{u}{u}_{k} n_{j}+P_{j}^{k}\left(-\omega_{k r} n^{r}+\sigma_{k r} n^{r}\right) n_{i} \\
& +P_{[i}^{k} P_{j]}^{r} \omega_{k r}+\left(P_{(i}^{k} P_{j)}^{r}-\frac{1}{2} P_{i j} P^{k r}\right) \sigma_{k r}+\frac{1}{2} P_{i j}\left(-\sigma_{k r} n^{k} n^{r}+\frac{2}{3} \theta\right)
\end{aligned}
$$

or in a matrix form

$$
\begin{aligned}
& u_{i ; j} \rightarrow \\
& \left(\begin{array}{ccc}
0 & 0 & 0 \\
-\dot{u}^{k} n_{k} & \left(\sigma_{r k} n^{r} n^{k}+\frac{1}{3} \theta\right) & P_{j}^{k}\left(-\omega_{k r} n^{r}+\sigma_{k r} n^{r}\right) \\
-P_{i}^{k} \dot{u}_{k} & P_{i}^{j}\left(\omega_{j k} n^{k}+\sigma_{j k} n^{k}\right) P_{[i}^{k} P_{j]}^{r} \omega_{k r}+\left(P_{(i}^{k} P_{j)}^{r}-\frac{1}{2} P_{i j} P^{k r}\right) \sigma_{k r}+\frac{1}{2} P_{i j}\left(-\sigma_{k r} n^{k} n^{r}+\frac{2}{3} \theta\right) .
\end{array}\right)
\end{aligned}
$$


15.1.2 The $1+1+2$ decomposition of the shear tensor $\sigma_{a b}$ and the vorticity tensor $\omega_{a b}$

For the stress and the vorticity tensors for the $1+1+2$ decomposition we find the result:

$$
\begin{aligned}
& \sigma_{i j}=\left(\sigma_{k r} n^{k} n^{r}\right)\left(n_{i} n_{j}-\frac{1}{2} P_{i j}\right)+2 P_{(i}^{k} n_{j)} \sigma_{k r} n^{r}+\left(P_{(i}^{k} P_{j)}^{r}-\frac{1}{2} P_{i j} P^{k r}\right) \sigma_{k r}, \\
& \omega_{i j}=2 P_{[i}^{k} n_{j]} \omega_{k r} n^{r}+\omega_{k r} P_{[i}^{k} P_{j]}^{r} .
\end{aligned}
$$

In matrix form, the above expressions are presented as follows:

$$
\begin{gathered}
\sigma_{i j} \rightarrow\left(\begin{array}{ccc}
0 & 0 & 0 \\
0 & \sigma_{k r} n^{k} n^{r} & P_{i}^{k} \sigma_{k r} n^{r} \\
0 & P_{j}^{k} \sigma_{k r} n^{r} & \left(P_{(i}^{k} P_{j)}^{r}-\frac{1}{2} P_{i j} P^{k r}\right)^{2} \sigma_{k r}-\frac{1}{2} P_{i j}\left(\sigma_{k r} n^{k} n^{r}\right)
\end{array}\right), \\
\omega_{i j} \rightarrow\left(\begin{array}{ccc}
0 & 0 & 0 \\
0 & 0 & -P_{i}^{k} \omega_{k r} n^{r} \\
-P_{j}^{k} \omega_{k r} n^{r} & \omega_{k r} P_{[i}^{k} P_{j]}^{r}
\end{array}\right)
\end{gathered}
$$

\subsubsection{The $1+1+2$ decomposition of the tensor $n_{a ; b}$}

We consider now the $1+1+2$ decomposition of the derivative $n_{a ; b}$ of the unit spacelike vector $n_{a}$ defining the double congruence. We find

$$
\begin{aligned}
& A_{00}: n_{i ; j} u^{i} u^{j}=\dot{n}^{i} u_{i}=-\dot{u}^{i} n_{i} \\
& A_{01}: n_{i ; j} u^{i} n^{j}=*^{i} u_{i}=-*^{*} n_{i}=-\left(\sigma_{r k} n^{r} n^{k}+\frac{1}{3} \theta\right) \\
& A_{10}: n_{i ; j} n^{i} u^{j}=0 \\
& A_{11}: n_{i ; j} n^{i} n^{j}=0 \\
& A_{i}: P_{i}^{j} n_{j ; k} u^{k}=P_{i}^{j} \dot{n}_{j} \\
& \widetilde{A_{i}}: P_{i}^{k} n_{j ; k} u^{j}=-P_{i}^{k} u_{j ; k} n^{j}=-P_{i}^{k}\left(\omega_{j k} n^{j}+\sigma_{j k} n^{j}\right) \\
& B_{i}: P_{i}^{j} n_{j ; k} n^{k}=P_{i}^{j} n_{j} \\
& \widetilde{B_{i}}: P_{j}^{k} n_{i ; k} n^{i}=0 \\
& \widetilde{C_{i j}}: P_{[i}^{k} P_{j]}^{r} n_{r ; k}=R_{i j} \\
& \widetilde{D_{i j}}:\left[P_{(i}^{k} P_{j}^{r}-\frac{1}{2} P_{i j} P^{k r}\right] n_{k ; r}=J_{i j} \\
& \widetilde{E}: \frac{1}{2} P_{i j} P^{k r} n_{k ; r}=\frac{1}{2} P_{i j} E,
\end{aligned}
$$

where we have set:

$$
P_{i}^{k} P_{j}^{r} n_{j ; r}=S_{i j}+R_{i j}+\frac{1}{2} E P_{i j},
$$

where

$$
\begin{aligned}
S_{i j} & =P_{i}^{k} P_{j}^{r} n_{(k ; r)}-\frac{1}{2} E P_{i j} \\
E & =P^{i j} n_{i ; j} \\
R_{i j} & =P_{i}^{k} P_{j}^{r} n_{[j ; r]} .
\end{aligned}
$$


We call $S_{i j}$ is the screen shear tensor, $R_{i j}$ the screen rotation tensor and $E$ the screen expansion of the vector field $n^{i}$.

From their definition, it is easy to show that the kinematic quantities of $n^{i}$ satisfy the properties:

$$
\begin{aligned}
R_{i j} & =-R_{j i}, R_{i j} n^{j}=0 \\
S_{i j} & =S_{j i}, S_{i}^{i}=0, S_{i j} n^{j}=0 \\
R_{i j} & =h_{i}^{k} h_{j}^{r} R_{k r} \\
S_{i j} & =h_{i}^{k} h_{j}^{r} S_{k r} .
\end{aligned}
$$

Coming back to the decomposition formula (218), we find

$$
\begin{aligned}
n_{i ; j}= & -\left(\dot{u}^{k} n_{k}\right) u_{i} u_{j}+\left(\sigma_{r k} n^{r} n^{k}+\frac{1}{3} \theta\right) u_{i} n_{j}-P_{i}^{k} \dot{n}_{k} u_{j}+P_{j}^{k}\left(\omega_{r k} n^{r}+\sigma_{j k} n^{j}\right) u_{i}+P_{i}^{k} n_{k}^{*} n_{j} \\
& +R_{i j}+J_{i j}+\frac{1}{2} P_{i j} E \\
= & -\left(\dot{u}^{k} n_{k}\right) u_{i} u_{j}+\left(\sigma_{r k} n^{r} n^{k}+\frac{1}{3} \theta\right) u_{i} n_{j}-P_{i}^{k} \dot{n}_{k} u_{j}+P_{j}^{k}\left(\omega_{r k} n^{r}\right) u_{i}+P_{j}^{k} \omega_{k r} n^{r} u_{i}+P_{i}^{k} n_{k} n_{j} \\
& +P_{j}^{k} \dot{n}_{k} u_{i}-N_{k} u_{i}+R_{i j}+J_{i j}+\frac{1}{2} P_{i j} E \\
= & -\left(\dot{u}^{k} n_{k}\right) u_{i} u_{j}+\left(\sigma_{r k} n^{r} n^{k}+\frac{1}{3} \theta\right) u_{i} n_{j}-P_{i}^{k} \dot{n}_{k} u_{j}+P_{j}^{k}\left(2 \omega_{r k} n^{r}-N_{k}\right) u_{i}+P_{i}^{k} n_{k}^{*} n_{j}+P_{j}^{k} \dot{n}_{k} u_{i} \\
& +R_{i j}+J_{i j}+\frac{1}{2} P_{i j} E
\end{aligned}
$$

where the Screen or Greenberg vector $N^{a}$ is defined as follows:

$$
N^{i}=P_{j}^{i}\left(\dot{n}^{j}-\stackrel{*}{u}^{j}\right)=P_{j}^{i} L_{n} u^{j}
$$

Finally, we have

$$
\begin{aligned}
n_{i ; j}= & -\left(\dot{u}^{k} n_{k}\right) u_{i} u_{j}+\left(\sigma_{r k} n^{r} n^{k}+\frac{1}{3} \theta\right) u_{i} n_{j}-P_{i}^{k} \dot{n}_{k} u_{j}+ \\
& +P_{j}^{k}\left(2 \omega_{j k} n^{j}-N_{j}\right) u_{i}+P_{i}^{k} n_{k}^{*} n_{j}+P_{j}^{k} \dot{n}_{k} u_{i}+R_{i j}+J_{i j}+\frac{1}{2} P_{i j} E
\end{aligned}
$$

and in matrix form:

$$
n_{i ; j} \rightarrow\left(\begin{array}{ccc}
-\dot{u}^{i} n_{i} & \left(\sigma_{r k} n^{r} n^{k}+\frac{1}{3} \theta\right) & P_{j}^{k} \dot{n}_{k}+P_{j}^{k}\left(2 \omega_{j k} n^{j}-N_{j}\right) \\
0 & 0 & 0 \\
-P_{i}^{k} \dot{n}_{k} & P_{i}^{k^{*}} n_{k} & R_{i j}+J_{i j}+\frac{1}{2} P_{i j} E .
\end{array}\right)
$$

The Greenberg vector is important because when $N^{a}=0$ the $L_{n} u^{j}$ is a linear combination of the vectors $u^{a}, n^{a}$ which is the condition that the integral curves of the vector fields $u^{a}, n^{a}$ form a surface. This condition is used in the RMHD approximation as the condition that the magnetic field is frozen in wrt the observers $u^{a}$, that is, a charge moves always on the same magnetic field line. 


\subsubsection{The $1+1+2$ decomposition of the energy-momentum tensor}

The energy-momentum tensor is a symmetric second rank tensor. We take $Y_{i j}=T_{i j}$ and find that the $1+1+2$ decomposition of this tensor ( for $u^{i} u_{i}=-1, n^{i} n_{i}=1$ and $\phi=0$ ) is as follows:

$$
\begin{aligned}
T_{i j}= & \mu u_{i} u_{j}+v n_{i} s_{j}+v s_{i} n_{j}+(p+\gamma) n_{i} n_{j}+Q_{j} u_{i} \\
& +P_{j} n_{i}+Q_{i} u_{j}+P_{i} n_{j}+D_{i j}+\left(p-\frac{1}{2} \gamma\right) P_{i j} .
\end{aligned}
$$

We know that the $1+3$ decomposition of the energy stress tensor wrt the vector $u^{i}$ is given by the expression:

$$
T_{i j}=\mu u_{i} u_{j}+p h_{i j}+2 u_{(i} q_{j)}+\pi_{i j},
$$

where

$$
q_{i} u^{i}=0, \pi_{i j} u^{j}=0, \pi_{i j}=\pi_{j i}, \pi_{i}^{i}=0 .
$$

The quantities of the $1+3$ decomposition are related to the quantities of the $1+1+2$ decomposition as follows:

$$
\begin{aligned}
q_{i} & =v n_{i}+Q_{i}, \\
\pi_{i j} & =\gamma\left(n_{i} n_{j}-\frac{1}{2} P_{i j}\right)+2 P_{(i} n_{j)}+D_{i j} .
\end{aligned}
$$

More on the $1+1+2$ decomposition of the energy stress tensor we shall mention below.

\subsubsection{The $1+1+2$ decomposition of the tensors $\left(\lambda u_{a}\right)_{; b}$ and $\left(\phi n_{a}\right)_{; b}$}

We shall need in our calculations the $1+1+2$ decomposition of the quantities $\left(\lambda u_{i}\right) ; j$ and $\left(\phi n_{i}\right) ; j$, where $\lambda, \phi$ are scalars (invariants). One derives easily the following results:

$$
\begin{aligned}
\left(\lambda u_{a}\right) ; b & =\lambda, b u_{a}+\lambda u_{a ; b} \\
& =\lambda_{, b} u^{b} u_{a} u_{b}+\lambda_{, b} n^{b} u_{a} n_{b}+P_{b}^{k} \lambda_{, k} u_{a}+\lambda u_{a ; b} \\
& =-\dot{\lambda} u_{a} u_{b}+\stackrel{*}{\lambda} u_{a} n_{b}+P_{b}^{k} \lambda,_{k} u_{a}+\lambda u_{a ; b}
\end{aligned}
$$

and

$$
\begin{aligned}
\left(\phi n_{a}\right)_{; b} & =\phi_{, b} n_{a}+\phi n_{a ; b} \\
& =-\phi, b u^{b} n_{a} u_{b}+\phi \phi_{, b} n^{b} n_{a} n_{b}+P_{b}^{k} \phi_{, k} n_{a}+\phi n_{a ; b} \\
& =-\dot{\phi} n_{a} u_{b}+\stackrel{*}{\phi} n_{a} n_{b}+P_{b}^{k} \phi_{, k} n_{a}+\phi n_{a ; b} .
\end{aligned}
$$

\subsubsection{The case of a general vector $\xi^{a}$}

We consider the vector field $\xi_{a}=-\lambda u_{a}+\phi n_{a}$, where $u_{a}, n_{a}$ are the vectors defining the double congruence. We calculate the $1+1+2$ decomposition of the covariant derivative $\xi_{a ; b}$ of the vector $\xi_{a}$ in the double congruence defined by the pair $u_{a}, n_{a}$. We have

$$
\xi_{a ; b}=-\left(\lambda u_{a}\right)_{; b}+\left(\phi n_{a}\right)_{; b},
$$

where we have computed $\left(\lambda u_{a}\right)_{; b},\left(\phi n_{a}\right)_{; b}$ in (239) and (240).

After some algebra, we find that

$$
\begin{aligned}
\xi_{a ; b}= & {\left[\dot{\lambda}-\phi \dot{u}^{k} n_{k}\right] u_{a} u_{b}+\left[-{ }^{*}+\phi\left(\sigma_{r k} n^{r} n^{k}+\frac{1}{3} \theta\right)\right] u_{a} n_{b}+\left[\lambda \dot{u}^{k} n_{k}-\dot{\phi}\right] n_{a} u_{b} } \\
& +\left[{ }^{*}-\lambda\left(\sigma_{a b} n^{a} n^{b}+\frac{1}{3} \theta\right)\right] n_{a} n_{b}
\end{aligned}
$$




$$
\begin{aligned}
& +\left[-\lambda, k+\phi \dot{n}_{k}+\phi\left(2 \omega_{b k} n^{b}-N_{b}\right)\right] u_{a} P_{b}^{k}+\left[\lambda \dot{u}_{k}-\phi \dot{n}_{k}\right] P_{a}^{k} u_{b} \\
& +\left[\phi_{, k}+\lambda\left(\omega_{k r} n^{r}-\sigma_{k r} n^{r}\right)\right] n_{a} P_{b}^{k}+\left[\phi \dot{n}_{k}^{*}-\lambda\left(\sigma_{k r}+\omega_{k r}\right) n^{r}\right] n_{b} P_{a}^{k} \\
& +\left[-\lambda \sigma_{k r}+\phi S_{a b}\right]\left(P_{(a}^{k} P_{b)}^{r}-\frac{1}{2} P_{a b} P^{k r}\right)+\left[\frac{1}{2} \lambda\left(\sigma_{k r} n^{k} n^{r}-\frac{2}{3} \theta\right)+\frac{1}{2} \phi E\right] P_{a b} \\
& +\left[-\lambda \omega_{k r}+\phi R_{k r}\right] P_{[a}^{k} P_{b]}^{r} .
\end{aligned}
$$

Having computed $\xi_{a ; b}$ we compute the symmetric and the antisymmetric part (for reasons to be seen later). For the symmetric part, we have

$$
\begin{aligned}
\xi_{(a ; b)}= & {\left.\left[\dot{\lambda}-\phi \dot{u}^{k} n_{k}\right] u_{a} u_{b}+\left[-{ }^{*}+\phi\left(\sigma_{r k} n^{r} n^{k}+\frac{1}{3} \theta\right)+\lambda \dot{u}^{k} n_{k}-\dot{\phi}\right] u_{(a} n_{b}\right) } \\
& +\left[\stackrel{*}{\phi}-\lambda\left(\sigma_{a b} n^{a} n^{b}+\frac{1}{3} \theta\right)\right] n_{a} n_{b} \\
& +\left[-\lambda, k+\phi\left(2 \omega_{b k} n^{b}-N_{b}\right)+\lambda \dot{u}_{k}\right] u_{(a} P_{b)}^{k} \\
& +\left[\phi_{, k}-2 \lambda \sigma_{k r} n^{r}+\phi n_{k}\right] n_{(a} P_{b)}^{k} \\
& +\left[-\lambda \sigma_{k r}+\phi S_{a b}\right]\left(P_{(a}^{k} P_{b)}^{r}-\frac{1}{2} P_{a b} P^{k r}\right) \\
& +\frac{1}{2}\left[\lambda\left(\sigma_{k r} n^{k} n^{r}-\frac{2}{3} \theta\right)+\phi E\right] P_{a b} .
\end{aligned}
$$

For the antisymmetric part $\xi_{[a ; b]}$, we find

$$
\begin{aligned}
\xi_{[a ; b]}= & {\left[-\lambda^{*}+\phi\left(\sigma_{r k} n^{r} n^{k}+\frac{1}{3} \theta\right)-\lambda \dot{u}^{k} n_{k}+\dot{\phi}\right] u_{[a} n_{b]} } \\
& +\left[-\lambda, k+2 \phi \dot{n}_{k}+\phi\left(2 \omega_{b k} n^{b}-N_{b}\right)-\lambda \dot{u}_{k}\right] u_{[a} P_{b]}^{k} \\
& +\left[\phi_{, k}+2 \lambda \omega_{k r} n^{r}-\phi \stackrel{n}{k}_{k}^{*}\right] n_{[a} P_{b]}^{k}+\left[-\lambda \omega_{k r}+\phi R_{k r}\right] P_{[a}^{k} P_{b]}^{r}
\end{aligned}
$$

Finally, the trace $\xi_{; a}^{a}$ is found to be

$$
\xi_{; a}^{a}=-\dot{\lambda}-\lambda \theta+\phi \dot{u}^{b} n_{b}+\phi E+\stackrel{*}{\phi}
$$

\section{Lie derivative and the $1+1+2$ decomposition}

As in the case of $1+3$ decomposition, we consider the $1+1+2$ decomposition of the Lie derivative of a vector and a second rank tensor to study the effects of a collineation in the kinematics and the dynamics of a model spacetime.

\section{$16.11+1+2$ decomposition wrt a double congruence}

Consider the double congruence defined by the timelike vector field $u^{i},\left(u^{i} u_{i}=-1\right)$ and a spacelike vector field $n^{i},\left(n^{i} n_{i}=1\right)$ and assume that $\phi=u^{i} n_{i}=0$.

Let $\xi^{i}$ be a vector field that is given by the expression:

$$
\xi^{i}=-\lambda u^{i}+\varphi n^{i}
$$

where $\lambda=\xi^{i} u_{i}$ and $\varphi=\xi^{i} n_{i}$. From the $1+1+2$ decomposition formula for vectors [see (217)], we have

$$
\begin{aligned}
\lambda_{; i} & =-\dot{\lambda} u_{i}+\lambda^{*} n_{i}+P_{i}^{j} \lambda_{; j} \\
\varphi_{; i} & =-\dot{\varphi} u_{i}+\varphi^{*} n_{i}+P_{i}^{j} \varphi_{; j} .
\end{aligned}
$$


Proposition 16.1 The following identities are true:

$$
\begin{aligned}
L_{\xi} u_{a}= & -\left[\dot{\lambda}-\phi \dot{u}^{k} n_{k}\right] u_{a}+\left[\dot{\lambda}^{*}-\lambda\left(\dot{u}^{k} n_{k}\right)\right] n_{a}+P_{a}^{k}\left[\lambda, k-\lambda \dot{u}_{k}-2 \phi \omega_{b k} n^{b}\right], \\
L_{\xi} u^{a}= & {\left[\dot{\lambda}-\phi \dot{u}^{k} n_{k}\right] u^{a}-\left[\dot{\phi}-\phi\left(\sigma_{c d} n^{c} n^{d}+\frac{1}{3} \theta\right)\right] n^{a}-\phi N^{a}, } \\
L_{\xi} n_{a}= & {\left[-\dot{\phi}+\phi\left(\sigma_{r k} n^{r} n^{k}+\frac{1}{3} \theta\right)\right] u_{a}+\left[\stackrel{*}{\phi}-\lambda\left(\sigma_{a b} n^{a} n^{b}+\frac{1}{3} \theta\right)\right] n_{a} } \\
& +P_{a}^{k}\left[\lambda\left(2 \omega_{t k} n^{t}-N_{k}\right)+2 \lambda \dot{n}_{k}-\phi \dot{n}_{k}^{*}-\phi_{; c}\right] \\
L_{\xi} n^{a}= & {\left[\dot{\lambda}-\lambda \dot{u}^{k} n_{k}\right] u^{a}-\left[\stackrel{*}{\phi}-\lambda\left(\sigma_{a b} n^{a} n^{b}+\frac{1}{3} \theta\right)\right] n^{a}-\lambda N^{a}, }
\end{aligned}
$$

where $N^{i}$ is the Greenberg or the screen vector which is given by the expression

$$
N^{i}=P_{j}^{i} L_{u} n^{j}=P_{j}^{i}\left(\dot{n}^{j}-\stackrel{*}{j}^{j}\right) .
$$

Proof We have for (248):

$$
\begin{gathered}
L_{\xi} u_{a}=u_{a ; b} \xi^{b}+\xi_{b ; a} u^{b} \\
\xi_{a ; b}=\left[\dot{\lambda}-\phi \dot{u}^{k} n_{k}\right] u_{a} u_{b}+\left[-\stackrel{*}{\lambda}+\phi\left(\sigma_{r k} n^{r} n^{k}+\frac{1}{3} \theta\right)\right] u_{a} n_{b}+\left[\lambda \dot{u}^{k} n_{k}-\dot{\phi}\right] n_{a} u_{b} \\
+\left[\dot{\phi}-\lambda\left(\sigma_{a b} n^{a} n^{b}+\frac{1}{3} \theta\right)\right] n_{a} n_{b} \\
+\left[-\lambda, k+\phi \dot{n}_{k}+\phi\left(2 \omega_{b k} n^{b}-N_{b}\right)\right] u_{a} P_{b}^{k}+\left[\lambda \dot{u}_{k}-\phi \dot{n}_{k}\right] P_{a}^{k} u_{b} \\
+\left[\phi, k+\lambda\left(\omega_{k r} n^{r}-\sigma_{k r} n^{r}\right)\right] n_{a} P_{b}^{k}+\left[\phi{ }^{*} n_{k}-\lambda\left(\sigma_{k r}+\omega_{k r}\right) n^{r}\right] n_{b} P_{a}^{k} \\
+\left[-\lambda \sigma_{k r}+\phi S_{a b}\right]\left(P_{(a}^{k} P_{b)}^{r}-\frac{1}{2} P_{a b} P^{k r}\right)+\left[\frac{1}{2} \lambda\left(\sigma_{k r} n^{k} n^{r}-\frac{2}{3} \theta\right)+\frac{1}{2} \phi E\right] P_{a b} \\
+\left[-\lambda \omega_{k r}+\phi R_{k r}\right] P_{[a}^{k} P_{b]}^{r} .
\end{gathered}
$$

The term

$$
\xi_{b ; a} u^{b}=-\left[\dot{\lambda}-\phi \dot{u}^{k} n_{k}\right] u_{a}+\left[\stackrel{\lambda}{*}^{*} \phi\left(\sigma_{r k} n^{r} n^{k}+\frac{1}{3} \theta\right)\right] n_{a}+\left[\lambda_{, k}-\phi \dot{n}_{k}-\phi\left(2 \omega_{b k} n^{b}-N_{k}\right)\right] P_{a}^{k},
$$

so that

$$
L_{\xi} u_{a}=u_{a ; b} \xi^{b}-\left[\dot{\lambda}-\phi \dot{u}^{k} n_{k}\right] u_{a}-\left[-\dot{\lambda}^{*}+\phi\left(\sigma_{r k} n^{r} n^{k}+\frac{1}{3} \theta\right)\right] n_{a}+\left[\lambda, k-\phi \dot{n}_{k}-\phi\left(2 \omega_{b k} n^{b}-N_{k}\right)\right] P_{a}^{k} .
$$

Similarly, from (220), we have

$$
u_{a ; b} \xi^{b}=-\lambda\left(\dot{u}^{k} n_{k}\right) n_{i}-\lambda P_{i}^{k} \dot{u}_{k}+\phi\left(\sigma_{r k} n^{r} n^{k}+\frac{1}{3} \theta\right) n_{i}+\phi P_{i}^{k} u_{k}^{*} .
$$

Collecting terms, we find

$$
L_{\xi} u_{a}=-\left[\dot{\lambda}-\phi \dot{u}^{k} n_{k}\right] u_{a}+\left[{ }^{*}-\lambda\left(\dot{u}^{k} n_{k}\right)\right] n_{a}+P_{a}^{k}\left[\lambda, k-\lambda \dot{u}_{k}-\phi 2 \omega_{b k} n^{b}\right] .
$$

We have for (249)

$$
L_{\xi} u^{a}=\left(L_{\xi} g^{a b}\right) u_{b}+g^{a b} L_{\xi} u_{b}=-2 \xi^{(a ; b)} u_{b}+g^{a b} L_{\xi} u_{b} .
$$


The first term gives

$$
\begin{aligned}
-2 \xi^{(a ; b)} u_{b}= & 2\left[\dot{\lambda}-\phi \dot{u}^{k} n_{k}\right] u^{a}+\left[-{ }^{*}+\phi\left(\sigma_{r k} n^{r} n^{k}+\frac{1}{3} \theta\right)+\lambda \dot{u}^{k} n_{k}-\dot{\phi}\right] n^{a} \\
& +\left[-\lambda_{, k}+\phi\left(2 \omega_{b k} n^{b}-N_{b}\right)+\lambda \dot{u}_{k}\right] P^{k a} .
\end{aligned}
$$

Replacing $L_{\xi} u_{b}$ from (248) and collecting terms, we find

$$
L_{\xi} u^{a}=\left[\dot{\lambda}-\phi \dot{u}^{k} n_{k}\right] u^{a}+\left[\phi\left(\sigma_{r k} n^{r} n^{k}+\frac{1}{3} \theta\right)-\dot{\phi}\right] n^{a}-\phi N^{a} .
$$

Concerning $L_{\xi} n_{a}$, we have

$$
L_{\xi} n_{a}=n_{a ; b} \xi^{b}+\xi_{b ; a} n^{b} .
$$

From (250), we find

$$
\xi_{b ; a} n^{b}=\left[\lambda \dot{u}^{k} n_{k}-\dot{\phi}\right] u_{a}+\left[\stackrel{*}{\phi}-\lambda\left(\sigma_{a b} n^{a} n^{b}+\frac{1}{3} \theta\right)\right] n_{a}+\left[\phi_{, k}+\lambda\left(\omega_{k r} n^{r}-\sigma_{k r} n^{r}\right)\right] P_{a}^{k} .
$$

Similarly, from (232), we have

$$
n_{a ; b} \xi^{b}=-\lambda\left(\dot{u}^{k} n_{k}\right) u_{j}-\lambda P_{j}^{k} \dot{n}_{k}+\phi\left(\sigma_{r k} n^{r} n^{k}+\frac{1}{3} \theta\right) u_{i}+\phi P_{i}^{k} \stackrel{n}{*}_{k}
$$

Replacing, we find

$$
\begin{aligned}
L_{\xi} n_{a}= & {\left[-\dot{\phi}+\phi\left(\sigma_{r k} n^{r} n^{k}+\frac{1}{3} \theta\right)\right] u_{a}+\left[\stackrel{*}{\phi}-\lambda\left(\sigma_{a b} n^{a} n^{b}+\frac{1}{3} \theta\right)\right] n_{a}+} \\
& -P_{a}^{k}\left[\lambda\left(2 \omega_{t k} n^{t}-N_{k}\right)+2 \lambda \dot{n}_{k}-\phi \stackrel{*}{n}_{k}-\phi_{; c}\right],
\end{aligned}
$$

where $N^{a}=P_{b}^{a} L_{n} u^{a}$ is the Greenberg vector.

Concerning $L_{\xi} n^{a}$, we have

$$
L_{\xi} n^{a}=\left(L_{\xi} g^{a b}\right) n_{b}+g^{a b} L_{\xi} n_{a}=-2 \xi^{(a ; b)} n_{b}+g^{a b} L_{\xi} n_{b} .
$$

Again from (250) we have for the first term:

$$
\begin{aligned}
-2 \xi^{(a ; b)} n_{b} & =-\left[-\lambda^{*}+\phi\left(\sigma_{r k} n^{r} n^{k}+\frac{1}{3} \theta\right) \lambda \dot{u}^{k} n_{k}-\dot{\phi}\right] u^{a}+ \\
& -2\left[{ }^{*} \phi-\lambda\left(\sigma_{a b} n^{a} n^{b}+\frac{1}{3} \theta\right)\right] n^{a}-\left[\phi_{, k}-2 \lambda \sigma_{k r} n^{r}+\phi n_{k}^{*}\right] P^{k a} .
\end{aligned}
$$

Using (250) and collecting terms, we find

$$
L \xi n^{a}=\left[\lambda^{*}-\lambda \dot{u}^{k} n_{k}\right] u^{a}-\left[\stackrel{*}{\phi}-\lambda\left(\sigma_{a b} n^{a} n^{b}+\frac{1}{3} \theta\right)\right] n^{a}-\lambda N^{a} .
$$

Proposition 16.2 Let $X^{i}$ be an arbitrary vector field and $u^{i}$ a unit timelike vector field. Then we have the identity

$$
L_{u} X^{i}=-\left(u^{r} u^{j} L_{u} g_{r j}\right) u^{i}+\left[\left(X^{j} n_{j}\right)^{\cdot}+2 R_{r j} X^{r} u^{j}-\phi_{; r} X^{r}\right] n^{i}+P_{j}^{i} L_{u} X^{i},
$$

where $\phi=u^{i} n_{i}$. 
Proof From Eq. (217), we have

$$
L_{u} X^{i}=-\left(\left(L_{u} X^{j}\right) u_{j}\right) u^{i}+\left(n_{j} L_{u} X^{j}\right) n^{i}+P_{j}^{i} L_{u} X^{j} .
$$

Contracting with $u^{i}, n^{i}$, we find

$$
\begin{aligned}
\left(L_{u} X^{j}\right) u_{j} & =X_{(j ; r)} u^{r} u^{i}=u^{r} u^{i} L_{u} g_{i j} \\
n_{j} L_{u} X^{j} & =\left(X^{j} n_{j}\right)^{\cdot}+2 n_{[j ; r]} X^{r} u^{j}-\phi_{; r} X^{r} .
\end{aligned}
$$

Hence,

$$
L_{u} X^{i}=-\left(u^{r} u^{j} L_{u} g_{r j}\right) u^{i}+\left[\left(X^{j} n_{j}\right)^{\cdot}+2 n_{[j ; r]} X^{r} u^{j}-\phi_{; r} X^{r}\right] n^{i}+P_{j}^{i} L_{u} X^{i} .
$$

Working in a similar manner, it is easy to prove the relations:

$$
\begin{aligned}
L_{n} X^{i} & =-\left[\left(X^{j} u_{j}\right)^{\cdot}+2 u_{[j ; r]} X^{r} u^{j}-\phi_{; r} X^{r}\right] u^{i}+\left(n^{r} n^{j} L_{u} g_{r j}\right) n^{i}+P_{j}^{i} L_{u} X^{i} \\
L_{u} n^{i} & =-\left(u^{r} u^{j} L_{u} g_{r j}\right) u^{i}+P_{j}^{i} n^{j} \\
L_{n} u^{i} & =\left(n^{r} n^{j} L_{u} g_{r j}\right) n^{i}+P_{j}^{i} u^{j} .
\end{aligned}
$$

16.2 The Lie derivative of the projection tensors

In the case $\xi^{a}=-\lambda u^{a}+\phi n^{a}$, we compute the Lie derivative of the projection tensors $h_{a b}=g_{a b}+u_{a} u_{a}$ and $P_{a b}=h_{a b}-n_{a} n_{a}$.

We have

$$
\begin{aligned}
L_{\xi} h_{a b}= & L_{\xi} g_{a b}+\left(L_{\xi} u_{a}\right) u_{b}+u_{a}\left(L_{\xi} u_{b}\right) \\
= & 2\left[-\dot{\phi}+\phi\left(\sigma_{r k} n^{r} n^{k}+\frac{1}{3} \theta\right)\right] u_{(a} n_{b)}-2 \phi N_{(b} u_{a)}+2\left[\stackrel{*}{\phi}-\lambda\left(\sigma_{a b} n^{a} n^{b}+\frac{1}{3} \theta\right)\right] n_{(a} n_{b)} \\
& +2\left[\phi,,_{c}-2 \lambda \sigma_{c d} n^{d}+\phi n_{c}^{*}\right] n_{(a} P_{b)}^{c} \\
& +2\left[-\lambda \sigma_{k r}+\phi S_{k r}\right]\left(P_{(a}^{k} P_{b)}^{r}-\frac{1}{2} P_{a b} P^{k r}\right)+\left[\lambda\left(\sigma_{k r} n^{k} n^{r}-\frac{2}{3} \theta\right)+\phi E\right] P_{a b} .
\end{aligned}
$$

We also have

$$
\begin{aligned}
L_{\xi} h_{b}^{a}= & \left(L_{\xi} u^{a}\right) u_{b}+u^{a}\left(L_{\xi} u_{b}\right) \\
= & {\left[-\dot{\phi}+\phi\left(\sigma_{r k} n^{r} n^{k}+\frac{1}{3} \theta\right)\right] n^{a} u_{b}+\left[{ }^{*}-\lambda \dot{u}^{k} n_{k}\right] u^{a} n_{b} } \\
& -\phi N^{a} u_{b}+P_{b}^{c}\left[\lambda_{; c}-\lambda \dot{u}_{c}-2 \phi \omega_{k c} n^{k}\right] u^{a}
\end{aligned}
$$

and

$$
\begin{aligned}
L_{\xi} h^{a b}= & L_{\xi} g^{a b}+\left(L_{\xi} u^{a}\right) u^{b}+u^{a}\left(L_{\xi} u^{b}\right) \\
= & 2\left[\stackrel{*}{\lambda}-\lambda \dot{u}^{k} n_{k}\right] n^{(a} u^{b)}+2 u^{(a} P^{b) c}\left[\lambda ; c-\lambda \dot{u}_{c}-2 \phi \omega_{d c} n^{d}\right] \\
& -2\left[\stackrel{*}{\phi}-\lambda\left(\sigma_{a b} n^{a} n^{b}+\frac{1}{3} \theta\right)\right] n^{a} n^{b}-2\left[\phi, c-2 \lambda \sigma_{c d} n^{d}+\phi n_{c}^{*}\right] n^{(a} P^{b) c} \\
& -\left[\lambda\left(\sigma_{k r} n^{k} n^{r}-\frac{2}{3} \theta\right)+\phi E\right] P^{a b}-2\left[-\lambda \sigma^{c d}+\phi S^{c d}\right]\left(P_{c}^{a} P_{d}^{b}-\frac{1}{2} P_{a b} P^{k r}\right) .
\end{aligned}
$$

Working in a similar manner, we compute the Lie derivative of the screen projection tensor $P_{a b}=h_{a b}-n_{a} n_{b}$. 
We find

$$
\begin{gathered}
L_{\xi} P_{a b}=L_{\xi} h_{a b}-2 L_{\xi} n_{(a} n_{b)} \\
=2 \lambda N_{(a} n_{b)}-2 \phi N_{(a} u_{b)}+2\left[-\lambda \sigma_{k r}+\phi S_{k r}\right]\left(P_{(a}^{k} P_{b)}^{r}-\frac{1}{2} P_{a b} P^{k r}\right) \\
+\left[\lambda\left(\sigma_{k r} n^{k} n^{r}-\frac{2}{3} \theta\right)+\phi E\right] P_{a b} \\
L_{\xi} P_{b}^{a}=L_{\xi} h_{b}^{a}-\left(L_{\xi} n^{a}\right) n_{b}-n^{a}\left(L_{\xi} n_{b}\right) \\
=\left[\lambda_{, k}-\lambda \dot{u}_{k}-2 \phi \omega_{r k} n^{r}\right] P_{b}^{k} u^{a}-\phi N^{a} u_{b}+\left[2 \lambda \sigma_{k d} n^{d}-\phi_{, k}-\phi n_{k}^{*}\right] P_{b}^{k} n^{a}+\lambda\left(N^{a} n_{b}+n^{a} N_{b}\right), \\
L_{\xi} P^{a b}=2\left[\phi N_{c}-2 \phi \omega_{t c} n^{t}-\lambda \dot{u}_{c}+\lambda ; c\right] u^{(a} P^{b) c}-2 \phi u^{(a} N^{b)} \\
-2\left[\phi_{; c}-2 \lambda \sigma_{c d} n^{d}+\phi n_{c}^{*}\right] n^{(a} P^{b) c}+2 \lambda n^{(a} N^{b)} \\
-2\left[\phi S^{c d}-\lambda \sigma^{c d}\right]\left(P_{(c}^{a} P_{d)}^{b}-\frac{1}{2} P_{c d} P^{a b}\right)-\left[\lambda\left(\sigma_{k r} n^{k} n^{r}-\frac{2}{3} \theta\right)+\phi E\right] P^{a b}
\end{gathered}
$$

16.3 The components of the generic symmetry in terms of the kinematic parameters

We express $L_{\xi} g_{a b}$ (the generic symmetry) in terms of the kinematic quantities of the double congruence. We introduce the trace and the trace-free part of the $L_{\xi} g_{a b}$ be means of the identity:

$$
L_{\xi} g_{a b}=2 \psi g_{a b}+2 H_{a b}, \quad H_{a}^{a}=0 \text { and } H_{[a b]}=0 .
$$

The quantities $\psi, H_{a b}$ we call the components of the generic symmetry.

Now for a general vector $\xi^{a}$, we have the decomposition:

$$
\xi_{a ; b}=\xi_{(a ; b)}+\xi_{[a ; b]} .
$$

But we know that

$$
2 \xi_{(a ; b)}=L_{\xi} g_{a b}
$$

therefore, we obtain

$$
\xi_{a ; b}=\psi g_{a b}+H_{a b}+\xi_{[a ; b]} .
$$

From (272), we compute the quantities $\psi$ and $H_{a b}$ in terms of the kinematic quantities of the double congruence. Indeed writing (272) in the form

$$
2 \xi_{(a ; b)}=L_{\xi} g_{a b}=2 \psi g_{a b}+2 H_{a b}
$$

and taking the trace, we find

$$
2 \xi_{; a}^{a}=8 \psi \Rightarrow \psi=\frac{1}{4}\left[-\dot{\lambda}-\lambda \theta+\phi \dot{u}^{b} n_{b}+\phi E+\stackrel{*}{\phi}\right],
$$

where we have used (247).

Concerning the tensor $H_{a b}$ we have from (273)

$$
H_{a b}=\xi_{(a ; b)}-\psi g_{a b} .
$$


Replacing $\xi_{(a ; b)}$ from (271) and $\psi$ from (274), we find after standard calculations:

$$
\begin{aligned}
H_{a b}= & \frac{1}{2}\left[3 \dot{\lambda}-3 \phi\left(\dot{u}^{k} n_{k}\right)-\lambda \theta+\phi E+\dot{\phi}^{*}\right] u_{a} u_{b} \\
& \left.+\left[-\dot{\lambda}+\phi\left(\sigma_{k r} n^{k} n^{r}+\frac{1}{3} \theta\right)+\lambda\left(\dot{u}^{k} n_{k}\right)-\dot{\phi}\right] u_{(a} n_{b}\right) \\
& +\frac{1}{2}\left[3 \stackrel{*}{\phi}-4 \lambda \sigma_{k r} n^{k} n^{r}-\frac{1}{3} \lambda \theta-\phi \dot{u}^{k} n_{k}-\phi E+\dot{\lambda}\right] n_{a} n_{b} \\
& +\left[-\lambda, k+\phi\left(2 \omega_{k r} n^{k}-N_{k}\right)+\lambda \dot{u}_{k}\right] u_{(a} P_{b)}^{r} \\
& +\left[\phi_{, k}-2 \lambda \sigma_{k r} n^{r}+\phi{ }^{*}{ }_{k}\right] n_{(a} P_{b)}^{k} \\
& +\left[-\lambda \sigma_{k r}+\phi S_{a b}\right]\left(P_{(a}^{k} P_{b)}^{r}-\frac{1}{2} P_{a b} P^{k r}\right) \\
& +\frac{1}{2}\left[\dot{\lambda}-\phi \dot{u}^{k} u_{k}-\frac{1}{3} \lambda \theta+2 \lambda \sigma_{k r} n^{k} n^{r}+\phi E-\stackrel{*}{\phi}\right] P_{a b} .
\end{aligned}
$$

In matrix form, this result is written as follows:

$$
H_{a b} \rightarrow\left(\begin{array}{rrr}
\frac{1}{2}\left[3 \dot{\lambda}-3 \phi\left(\dot{u}^{k} n_{k}\right)-\lambda \theta+\phi E+\stackrel{*}{\phi}\right] & \frac{1}{2}\left[-{ }^{*}+\phi\left(\sigma_{k r} n^{k} n^{r}+\frac{1}{3} \theta\right)+\lambda\left(\dot{u}^{k} n_{k}\right)-\dot{\phi}\right] & H_{13} \\
& \frac{1}{2}\left[3 \stackrel{*}{\phi}-4 \lambda \sigma_{k r} n^{k} n^{r}-\frac{1}{3} \lambda \theta-\phi \dot{u}^{k} n_{k}-\phi E+\dot{\lambda}\right] & H_{23} \\
H_{33}
\end{array}\right),
$$

where

$$
\begin{aligned}
& H_{13}=\frac{1}{2}\left[-\lambda, k+\phi\left(2 \omega_{k r} n^{k}-N_{k}\right)+\lambda \dot{u}_{k}\right] P_{b}^{r} \\
& H_{23}=\frac{1}{2}\left[\phi_{, k}-2 \lambda \sigma_{k r} n^{r}+\phi n_{k}^{*}\right] P_{b}^{k} \\
& H_{33}=\left[-\lambda \sigma_{k r}+\phi S_{a b}\right]\left(P_{(a}^{k} P_{b)}^{r}-\frac{1}{2} P_{a b} P^{k r}\right)+\frac{1}{2}\left[\dot{\lambda}-\phi \dot{u}^{k} u_{k}-\frac{1}{3} \lambda \theta+2 \lambda \sigma_{k r} n^{k} n^{r}+\phi E-\stackrel{*}{\phi}\right] P_{a b} .
\end{aligned}
$$

These expressions can be used to answer the question: given $\xi^{a}, n^{a}$, what type of collineation $\xi^{a}$ can be and under what conditions? Below we express the kinematic quantities of the double congruence in terms of the symmetry parameters $\psi, H_{a b}$.

\subsection{The kinematic implications of a collineation}

The following theorem gives the kinematic implications of a collineation, that is, expresses the kinematic quantities in terms of the collineation parameters $\psi, H_{a b}$ in the $1+1+2$ decomposition.

Theorem 16.3 Suppose $\xi^{i}=\xi n^{i}, n^{i} n_{i}=\varepsilon(n)$ and $s^{i} s_{i}=\varepsilon(s)$ are two non-null normalized vector fields such that $s^{i} n_{i}=\phi$, where $\varepsilon(s), \varepsilon(n)= \pm 1$ are the signatures of the vectors, the sign + applying to a spacelike vector and the - sign to a timelike vector. Let $P_{i j}=P_{i j}(s, n)$ be the projective tensor associated with the double congruence consisting of the vector fields $s^{i}$ and $n^{i}$. Then Eq. (230) is equivalent to the following conditions:

$$
\begin{aligned}
S_{i j} & =\frac{1}{\xi}\left[P_{i}^{k} P_{j}^{r}-\frac{1}{2} P^{k r} P_{i j}\right] H_{k r} \\
\dot{n}^{i} s_{i} & =\frac{1}{\xi} \varepsilon(s) \psi-\phi(\ln \xi)^{\bullet}+\frac{1}{\xi} H_{11}
\end{aligned}
$$




$$
\begin{aligned}
n_{i}^{*}= & \frac{\phi n_{i}-\varepsilon(n) s_{i}}{\phi^{2}-\varepsilon(s) \varepsilon(n)}\left[\frac{1}{\xi} \phi \psi-\varepsilon(n)(\ln \xi)^{\bullet}+\frac{2}{\xi} H_{21}-\frac{\varepsilon(n)}{\xi} \phi H_{22}\right]+ \\
& +P_{i}^{j}\left[-\varepsilon(n)(\ln \xi)_{; j}+\frac{2}{\xi} H_{j 2}\right] \\
\xi^{*}= & \psi+\varepsilon(n) H_{22} \\
E= & \frac{2 \psi}{\xi}+\frac{1}{\xi} P^{i j} H_{i j} \\
N_{i}= & -2 \omega_{i j} n^{j}-P_{i}^{j} \phi[\ln (\xi|\phi|)]_{; b}+\frac{2}{\xi} P_{i}^{j} H_{j 1}-\varepsilon(s) \phi P_{i}^{j} \dot{s}_{j},
\end{aligned}
$$

where $\dot{v}=v_{; k} s^{k}, v^{*}=v_{; k} n^{k}$ and $N_{i}=\left(n_{i ; j} u^{j}-u_{i ; j} n^{j}\right)=\left(\dot{n}_{i}-u_{i}^{*}\right)$ is the Greenberg vector.

Corollary 16.4 Let $\xi^{i}=\xi n^{i}, n^{i} n_{i}=\varepsilon(n)$ be a CKV and $s^{i}, s^{i} s_{i}=\varepsilon(s)$ be a unit vector such that $n^{i} s_{i}=\phi$. Then the conformal symmetry condition $L_{\xi} g_{i j}=2 \psi g_{i j}$ is equivalent to the conditions:

$$
\begin{aligned}
S_{i j} & =0 \\
\dot{n}^{i} s_{i} & =\frac{1}{\xi}\left[\varepsilon(s) \psi-\phi(\ln \xi)^{\bullet}\right] \\
n_{i}^{*} & =\frac{1}{\phi^{2}-\varepsilon(s) \varepsilon(n)}\left(\phi n_{i}-\varepsilon(n) s_{i}\right)\left[\frac{1}{\xi} \phi \psi-\varepsilon(n)(\ln \xi)^{\bullet}\right]-\varepsilon(n) P_{i}^{j}(\ln \xi)_{; j} \\
\xi^{*} & =\psi \\
E & =\frac{2 \psi}{\xi} \\
N_{i} & =-2 \omega_{i j} n^{j}-P_{i}^{j} \phi[\ln (\xi|\phi|)]_{; b}-\varepsilon(s) \phi P_{i}^{j} \dot{s}_{j} .
\end{aligned}
$$

Corollary 16.5 In the special case $n^{i} n_{i}=1, s^{i} s_{i}=-1, n^{i} s_{i}=0$, we have that $\xi^{i}=\xi n^{i}$ is a spacelike CKV iff:

$$
\begin{aligned}
S_{i j} & =0 \\
\dot{n}^{i} s_{i} & =-\frac{\psi}{\xi} \\
n_{i}^{*} & =s_{i}(\ln \xi)^{\bullet}-P_{i}^{j}(\ln \xi)_{; j} \\
\xi^{*} & =\psi \\
E & =\frac{2 \psi}{\xi} \\
N_{i} & =-2 \omega_{i j} n^{j} .
\end{aligned}
$$

\section{The Lie derivative of $R_{a b}$ wrt a collineation $\xi_{a}$}

In this section, we compute the Lie derivative of the Einstein equations in the $1+1+2$ decomposition. To do that, we write these equations in the form:

$$
R_{a b}=T_{a b}-\frac{1}{2} g_{a b} T+\Lambda g_{a b}
$$

The Lie derivative of Einstein field equations wrt a general vector field ${ }^{23} \xi^{a}$ is

$$
L_{\xi} R_{a b}=L_{\xi}\left[T_{a b}-\frac{1}{2} g_{a b} T+\Lambda g_{a b}\right]
$$

\footnotetext{
${ }^{23}$ A vector $\xi^{a}$ can always be written as $\xi^{a}=-\lambda u^{a}+\phi n^{a}$, where $n^{a} / / h_{b}^{a} \xi^{b}$.
} 
The LHS $L_{\xi} R_{a b}$ is of a pure geometric nature and can be computed in terms of the generic symmetry $L_{\xi} g_{a b}$ or, in terms of the geometric parameters $\psi, H_{a b}$. If we do that then we shall have expressed the Lie derivative of energy-momentum tensor in terms of geometric variables. Because the generic symmetry describes any symmetry, this means that by doing this we have solved Einstein field equations for the symmetry defined by the symmetry parameters $\psi, H_{a b}$.

We consider next the dynamic variables $\mu, p, q^{a}, \pi_{a b}$ defined by the $1+3$ decomposition of the energy momentum tensor wrt any observers $u^{a}$. As we have shown (see Eq. (128) ) the $1+3$ decomposition of the energy momentum tensor wrt the four-velocity $u^{a}$ is given by the relation:

$$
\begin{aligned}
T_{a b} & =\mu u_{a} u_{b}+p h_{a b}+q_{a} u_{b}+q_{b} u_{a}+\pi_{a b} \\
T & =g_{a b} T^{a b}=3 p-\mu .
\end{aligned}
$$

Replacing in Eq. (296), we find

$$
R_{a b}=(\mu+p) u_{a} u_{b}+\frac{1}{2}(\mu-p+2 \Lambda) g_{a b}+q_{a} u_{b}+q_{b} u_{a}+\pi_{a b}
$$

or

$$
R_{a b}=\left(\frac{1}{2} \mu+\frac{3}{2} p-\Lambda\right) u_{a} u_{b}+\left(\frac{1}{2} \mu-\frac{1}{2} p+\Lambda\right) h_{a b}+q_{a} u_{b}+q_{b} u_{a}+\pi_{a b} .
$$

In the $1+1+2$ decomposition wrt a double congruence $u^{a}, n^{a}$, we have shown (see Eq. (235)) that

$$
\begin{aligned}
T_{a b}= & \mu u_{a} u_{b}+v n_{a} u_{b}+v u_{a} n_{b}+(p+\gamma) n_{a} n_{b}+Q_{b} u_{a} \\
& +P_{b} n_{a}+Q_{a} u_{b}+P_{a} n_{b}+D_{a b}+\left(p-\frac{1}{2} \gamma\right) P_{a b},
\end{aligned}
$$

where

$$
\begin{aligned}
q_{a} u^{a} & =0, \pi_{a b} u^{b}=0, \pi_{a b}=\pi_{b a}, \pi_{a}^{a}=0 \\
q_{a} & =v n_{a}+Q_{a} \\
\pi_{a b} & =\gamma\left(n_{a} n_{b}-\frac{1}{2} P_{a b}\right)+2 P_{(a} n_{b)}+D_{a b} .
\end{aligned}
$$

Therefore, for this decomposition the Ricci tensor reads:

$$
\begin{aligned}
R_{a b}= & \left(\frac{1}{2} \mu+\frac{3}{2} p-\Lambda\right) u_{a} u_{b}+v n_{a} u_{b}+v u_{a} n_{b}+\left(\frac{1}{2} \mu-\frac{1}{2} p+\gamma+\Lambda\right) n_{a} n_{b} \\
& +Q_{b} u_{a}+P_{b} n_{a}+Q_{a} u_{b}+P_{a} n_{b}+D_{a b}+\left(\frac{1}{2} \mu-\frac{1}{2} p-\frac{1}{2} \gamma+\Lambda\right) P_{a b} .
\end{aligned}
$$

The conclusion is that by working in this manner we will express the field equations as Lie derivatives of the dynamic variables either in the $1+3$ or in the $1+1+2$ decomposition in terms of the geometric parameters $\psi, H_{a b}$. defining the symmetry vector $\xi^{a}$.

Before we compute the Lie derivative $L_{\xi} R_{a b}$ we note that any vector field $\xi^{a}$ can be written as $\xi^{a}=$ $-\lambda u^{a}+\phi n^{a}$ (where $n^{a}|| h_{b}^{a} \xi^{b}$ ); therefore, by the linearity of the Lie derivative, we have

$$
\begin{aligned}
L_{\xi} R_{a b}= & L_{-\lambda u} R_{a b}+L_{\phi n} R_{a b} \\
= & {\left[R_{a b ; c}\left[-\lambda u^{c}\right]+R_{c b}\left[-\lambda u^{c}\right]_{; a}+R_{a c}\left[-\lambda u^{c}\right]_{; b}\right] } \\
& +\left[R_{a b ; c}\left[\phi n^{c}\right]+R_{c b}\left[\phi n^{c}\right]_{; a}+R_{a c}\left[\phi n^{c}\right]_{; b}\right] \\
= & {\left[-\lambda \dot{R}_{a b}+R_{c b}\left[-\lambda u^{c}\right]_{; a}+R_{a c}\left[-\lambda u^{c}\right]_{; b}\right] } \\
& +\left[\phi R_{a b}+R_{c b}\left[\phi n^{c}\right]_{; a}+R_{a c}\left[\phi n^{c}\right]_{; b}\right] .
\end{aligned}
$$

This implies that we should brake the calculation of $L_{\xi} R_{a b}$ in three steps. First, we calculate the Lie derivative wrt a spacelike vector $\xi^{a}=\xi n^{a}$, then wrt a timelike vector $\xi^{a}=\xi u^{a}$ and finally wrt the general vector $\xi^{a}=-\lambda u^{a}+\phi n^{a}$. 
Before we start the calculation we recall the relation:

$$
L_{\xi} R_{a b}=R_{a b ; c} \xi^{c}+R_{c b} \xi_{; a}^{c}+R_{a c} \xi_{; a}^{c}=\stackrel{\circ}{R}_{a b}+2 R_{c(a} \xi_{; b)}^{c},
$$

where $\stackrel{\circ}{R}_{a b}=R_{a b ; c} \xi^{c}$ and a o over a symbol means covariant differentiation wrt $\xi^{a}$.

$17.11+1+2$ decomposition of the terms $\stackrel{\circ}{R}_{a b}, 2 R_{c(a} \xi_{; b)}^{c}$

For the term $\stackrel{\circ}{R}_{a b}$, we have

$$
\begin{aligned}
& R_{a b}=\left(\frac{1}{2} \mu+\frac{3}{2} p-\Lambda\right) u_{a} u_{b}+v n_{a} u_{b}+v u_{a} n_{b}+\left(\frac{1}{2} \mu-\frac{1}{2} p+\gamma+\Lambda\right) n_{a} n_{b}+ \\
& +Q_{b} u_{a}+P_{b} n_{a}+Q_{a} u_{b}+P_{a} n_{b}+D_{a b}+\left(\frac{1}{2} \mu-\frac{1}{2} p-\frac{1}{2} \gamma+\Lambda\right) P_{a b} \Rightarrow \\
& \left.\stackrel{\circ}{R}_{a b}=\left(\frac{1}{2} \mu+\frac{3}{2} p-\Lambda\right)^{\circ} u_{a} u_{b}+\left(\frac{1}{2} \mu+\frac{3}{2} p-\Lambda\right) 2 \stackrel{\circ}{u}_{(a} u_{b)}+2 v\left[\stackrel{\circ}{n}_{(a} u_{b)}+n_{(a} \stackrel{\circ}{u}\right)_{b}\right] \\
& +\left(\frac{1}{2} \mu-\frac{1}{2} p+\gamma+\Lambda\right)^{\circ} n_{a} n_{b}+\left(\frac{1}{2} \mu-\frac{1}{2} p+\gamma+\Lambda\right) 2 \stackrel{\circ}{(a}_{(a} n_{b)}+\left(\frac{1}{2} \mu-\frac{1}{2} p-\frac{1}{2} \gamma+\Lambda\right)^{\circ} P_{a b} \\
& +\left(\frac{1}{2} \mu-\frac{1}{2} p-\frac{1}{2} \gamma+\Lambda\right) 2 \stackrel{\circ}{u}_{(a} u_{b)}-\left(\frac{1}{2} \mu-\frac{1}{2} p-\frac{1}{2} \gamma+\Lambda\right) 2 \stackrel{\circ}{(a}_{(a} n_{b)},
\end{aligned}
$$

finally,

$$
\begin{aligned}
& \left.\stackrel{\circ}{R}_{a b}=\frac{1}{2}(\mu+3 p)^{\circ} u_{a} u_{b}+2\left(\mu+p-\frac{1}{2} \gamma\right) \stackrel{\circ}{u}_{(a} u_{b}\right) \\
& +\left(\frac{1}{2} \mu-\frac{1}{2} p+\gamma\right)^{\circ} n_{a} n_{b}+2 \frac{3}{2} \gamma \stackrel{\circ}{(a}_{(a)} n_{b)}+2 i n_{(a} u_{b)}+ \\
& +2 v\left(\stackrel{\circ}{n}_{(a} u_{b)}+n_{(a} \stackrel{\circ}{u}_{b)}\right)+2 \stackrel{\circ}{Q}_{(b} u_{a)}+2 Q_{(b} \stackrel{\circ}{u}_{a)} \\
& +2 \stackrel{\circ}{P}_{(b} n_{a)}+2 P_{(b} \stackrel{\circ}{n}_{a)}+\stackrel{\circ}{D}_{a b}+\left(\frac{1}{2} \mu-\frac{1}{2} p-\frac{1}{2} \gamma\right)^{\circ} P_{a b} \text {. }
\end{aligned}
$$

From this we compute the quantities

$$
\begin{aligned}
& u_{a} u_{b}: \stackrel{\circ}{R}_{a b} u^{a} u^{b}=\frac{1}{2}(\mu+3 p)^{\circ}+2 v \stackrel{\circ}{u}_{c} n^{c}-2 \stackrel{\circ}{Q}_{c} u^{c} \\
& u_{a} n_{b}: \stackrel{\circ}{R}_{a b} u^{a} n^{b}=-(\mu+p+\gamma) \stackrel{\circ}{u}_{c} n^{c}-\stackrel{\circ}{v}-\stackrel{\circ}{Q}_{c} n^{c}-P^{c} \stackrel{\circ}{u}_{c} \\
& u_{a} P_{b}^{c}: \stackrel{\circ}{R}_{a c} u^{a} P_{b}^{c}=-\left(\mu+p-\frac{1}{2} \gamma\right) \stackrel{\circ}{u}_{d} P_{c}^{d}-\left(v \stackrel{\circ}{d}_{d}+\stackrel{\circ}{Q}_{d}\right) P_{c}^{d}-P_{d}\left(\stackrel{\circ}{u}_{k} n^{k}\right) P_{c}^{d}-D_{k d} \stackrel{\circ}{k}^{k} P_{c}^{d} \\
& n_{a} n_{b}: \stackrel{\circ}{R}_{a b} n^{a} n^{b}=\left(\frac{1}{2} \mu-\frac{1}{2} p+\gamma\right)^{\circ}+2 v \stackrel{\circ}{c}_{c} n^{c}-2 P_{k} \stackrel{\circ}{k}^{k} \\
& n_{a} P_{b}^{c}: \stackrel{\circ}{R}_{a c} n^{a} P_{b}^{c}=v \stackrel{\circ}{u}_{d} P_{c}^{d}+\left(\stackrel{\circ}{u}_{k} n^{k}\right) Q_{d} P_{c}^{d}+\stackrel{\circ}{P}_{d} P_{c}^{d}+\frac{3}{2} \gamma \stackrel{\circ}{n}_{c}-D_{d c} \stackrel{\circ}{n}^{d} \\
& \left.P_{a}^{c} P_{b}^{d}: \stackrel{\circ}{R}_{c d} P_{a}^{c} P_{b}^{d}=\frac{1}{2}(\mu-p-\gamma)^{\circ} P_{c d}+2 Q_{(k} \stackrel{\circ}{u}\right)_{r} P_{c}^{k} P_{d}^{r}+2 P_{(c} \stackrel{\circ}{n}_{d)}+\stackrel{\circ}{D}_{k r} P_{c}^{k} P_{d}^{r}
\end{aligned}
$$


which lead to the $1+1+2$ decomposition

$$
\begin{aligned}
& \left.\stackrel{\circ}{R}_{a b}=\left[\frac{1}{2}(\mu+3 p)^{\circ}+2 v \stackrel{\circ}{u}_{c} n^{c}-2 \stackrel{\circ}{Q}_{c} u^{c}\right] u_{a} u_{b}-2\left[-(\mu+p+\gamma) \stackrel{\circ}{u}_{c} n^{c}-\stackrel{\circ}{v}-\stackrel{\circ}{Q}_{c} n^{c}-P^{c} \stackrel{\circ}{u}_{c}\right] u_{(a} n_{b}\right) \\
& -2\left[-\left(\mu+p-\frac{1}{2} \gamma\right) \stackrel{\circ}{u}_{d} P_{c}^{d}-\left(v \stackrel{\circ}{d}_{d}+\stackrel{\circ}{d}_{d}\right) P_{c}^{d}-P_{d}\left(\stackrel{\circ}{k}_{k} n^{k}\right) P_{c}^{d}-D_{k d} \dot{\varkappa}^{k} P_{c}^{d}\right] u_{(a} P_{b)}^{c} \\
& +\left[\left(\frac{1}{2} \mu-\frac{1}{2} p+\gamma\right)^{\circ}+2 v \stackrel{\circ}{c}_{c} n^{c}-2 P_{k} \stackrel{\circ}{n}^{k}\right] n_{a} n_{b} \\
& +2\left[v{\stackrel{\circ}{u_{d}}}_{c}^{d}+\left(\stackrel{\circ}{u}_{k} n^{k}\right) Q_{d} P_{c}^{d}+\stackrel{\circ}{P}_{d} P_{c}^{d}+\frac{3}{2} \gamma \stackrel{\circ}{n}_{c}-D_{d c} \stackrel{\circ}{n}^{d}\right] n_{(a} P_{b)}^{c}+ \\
& +\frac{1}{2}(\mu-p-\gamma)^{\circ} P_{c d}+2 Q_{(k} \stackrel{\circ}{u}_{r)} P_{c}^{k} P_{d}^{r}+2 P_{(c} \stackrel{\circ}{n}_{d)}+\stackrel{\circ}{D}_{k r} P_{c}^{k} P_{d}^{r} .
\end{aligned}
$$

For the term $R_{a c} \xi_{; b}^{c}$, we have

$$
\begin{aligned}
R_{a c} \xi_{; b}^{c}= & \left(\frac{1}{2} \mu+\frac{3}{2} p-\Lambda\right) u_{a} u_{c} \xi_{; b}^{c}+v n_{a} u_{c} \xi_{; b}^{c}+v u_{a} n_{c} \xi_{; b}^{c}+\left(\frac{1}{2} \mu-\frac{1}{2} p+\gamma+\Lambda\right) n_{a} n_{c} \xi_{; b}^{c} \\
& +Q_{c} \xi_{; b}^{c} u_{a}+P_{c} \xi_{; b}^{c} n_{a}+Q_{a} u_{c} \xi_{; b}^{c}+P_{a} n_{c} \xi_{; b}^{c}+D_{a c} \xi_{; b}^{c}+\left(\frac{1}{2} \mu-\frac{1}{2} p-\frac{1}{2} \gamma+\Lambda\right) P_{a c} \xi_{; b}^{c}
\end{aligned}
$$

We compute the contractions

$$
\begin{aligned}
& u_{a} u_{b}: R_{a c} \xi_{; b}^{c}=-\frac{1}{2}(\mu+3 p-2 \Lambda)\left(\dot{\xi}_{c} u^{c}\right)-v n_{c} \dot{\xi}^{c}-Q_{c} \dot{\xi}^{c} \\
& u_{a} n_{b}: R_{a c} \xi_{; b}^{c}=-\frac{1}{2}(\mu+3 p-2 \Lambda) u_{c}{\stackrel{*^{c}}{\xi}}^{c}-v n_{c} \stackrel{*}{\xi}^{c}-Q_{c} \xi^{*^{c}} \\
& n_{a} u_{b}: R_{a c} \xi_{; b}^{c}=\frac{1}{2}(\mu-p+2 \gamma+2 \Lambda) \dot{\xi}_{c} n^{c}+v\left(\dot{\xi}^{c} u_{c}\right)+P_{c} \dot{\xi}^{c} \\
& u_{a} P_{b}^{c}: R_{a c} \xi_{; b}^{c}=\frac{1}{2}(\mu+3 p-2 \Lambda) u_{k} \xi_{; d}^{k} P_{c}^{d}-v n_{k} \xi_{; d}^{k} P_{c}^{d}+Q_{k} \xi_{; d}^{k} P_{c}^{d} \\
& P_{a}^{c} u_{b}: R_{a c} \xi_{; b}^{c}=\frac{1}{2}(\mu-p-\gamma+2 \Lambda) \dot{\xi}_{d} P_{c}^{d}+Q_{d}\left(\dot{\xi}^{k} u_{k}\right) P_{c}^{d}+P_{d}\left(n_{k} \dot{\xi}^{k}\right) P_{c}^{d}+D_{d k} \dot{\xi}^{k} P_{c}^{d} \\
& \left.n_{a} n_{b}: R_{a c} \xi_{; b}^{c}=\frac{1}{2}(\mu-p+2 \gamma+2 \Lambda) \stackrel{*}{\xi}_{c} n^{c}\right)+v\left(u^{c} \xi_{c}^{*}\right)+P^{c} \xi_{c}^{*} \\
& n_{a} P_{b}^{c}: R_{a c} \xi_{; b}^{c}=\frac{1}{2}(\mu-p+2 \gamma+2 \Lambda) n_{k} \xi_{; d}^{k} P_{c}^{d}+v u_{c} \xi_{; d}^{c} P_{e}^{d}+P_{c} \xi_{; d}^{c} P_{e}^{d} \\
& P_{a}^{c} n_{b}: R_{a c} \xi_{; b}^{c}=\frac{1}{2}(\mu-p-\gamma+2 \Lambda) \stackrel{\xi}{\xi}_{c}+Q_{c}\left(u^{k} \xi_{k}^{*}\right)+P_{d} n_{k} \stackrel{*}{\xi}^{k} P_{c}^{d}+D_{d k} \stackrel{*}{\xi}^{k} P_{c}^{d} \\
& P_{a}^{c} P_{b}^{d}: R_{a c} \xi_{; b}^{c}=\frac{1}{2}(\mu-p-\gamma+2 \Lambda) \xi_{k ; r} P_{c}^{k} P_{d}^{r}+Q_{c}\left(u_{k} \xi_{; d}^{k}\right)+P_{c}\left(n_{k} \xi_{; d}^{k}\right)+D_{c k} \xi_{; d}^{k}
\end{aligned}
$$

from which follows:

$$
\begin{aligned}
R_{a c} \xi_{; b}^{c}= & {\left[-\frac{1}{2}(\mu+3 p-2 \Lambda)\left(\dot{\xi}_{c} u^{c}\right)-v n_{c} \dot{\xi}^{c}-Q_{c} \dot{\xi}^{c}\right] u_{a} u_{b} } \\
& -\left[-\frac{1}{2}(\mu+3 p-2 \Lambda) u_{c} \stackrel{\xi}{\xi}^{c}-v n_{c} \dot{\xi}^{c}-Q_{c} \dot{*}^{c}\right] u_{a} n_{b} \\
& -\left[\frac{1}{2}(\mu-p+2 \gamma+2 \Lambda) \dot{\xi}_{c} n^{c}+v\left(\dot{\xi}^{c} u_{c}\right)+P_{c} \dot{\xi}^{c}\right] u_{b} n_{a} \\
& -\left[\frac{1}{2}(\mu+3 p-2 \Lambda) u_{k} \xi_{; d}^{k} P_{c}^{d}-v n_{k} \xi_{; d}^{k} P_{c}^{d}+Q_{k} \xi_{; d}^{k} P_{c}^{d}\right] u_{a} P_{b}^{c} \\
& -\left[\frac{1}{2}(\mu-p-\gamma+2 \Lambda) \dot{\xi}_{d} P_{c}^{d}+Q_{d}\left(\dot{\xi}^{k} u_{k}\right) P_{c}^{d}+P_{d}\left(n_{k} \dot{\xi}^{k}\right) P_{c}^{d}+D_{d k} \dot{\xi}^{k} P_{c}^{d}\right] u_{b} P_{a}^{c}
\end{aligned}
$$




$$
\begin{aligned}
& +\left[\frac{1}{2}(\mu-p+2 \gamma+2 \Lambda)\left(_{\xi_{c}}^{*} n^{c}\right)+v\left(u^{c} \xi_{c}^{*}\right)+P^{c} \xi_{c}^{*}\right] n_{a} n_{b} \\
& +\left[\frac{1}{2}(\mu-p+2 \gamma+2 \Lambda) n_{k} \xi_{; d}^{k} P_{c}^{d}+v u_{c} \xi_{; d}^{c} P_{e}^{d}+P_{c} \xi_{; d}^{c} P_{e}^{d}\right] n_{a} P_{b}^{c} \\
& +\left[\frac{1}{2}(\mu-p-\gamma+2 \Lambda) \xi_{d}^{*} P_{c}^{d}+Q_{c}\left(u^{k} \xi_{k}^{*}\right)+P_{d} n_{k} \xi^{* k} P_{c}^{d}+D_{d k} \xi^{* k} P_{c}^{d}\right] n_{b} P_{a}^{c} \\
& +\left[\frac{1}{2}(\mu-p-\gamma+2 \Lambda) \xi_{k ; r} P_{c}^{k} P_{d}^{r}+Q_{c}\left(u_{k} \xi_{; d}^{k}\right)+P_{c}\left(n_{k} \xi_{; d}^{k}\right)+D_{c k} \xi_{; d}^{k}\right] P_{a}^{c} P_{b}^{d} .
\end{aligned}
$$

Calculation of the term $R_{c b} \xi_{; a}^{c}$.

We have

$$
\begin{aligned}
R_{c b} \xi_{; a}^{c}= & {\left[-\frac{1}{2}(\mu+3 p-2 \Lambda)\left(\dot{\xi}_{c} u^{c}\right)-v n_{c} \dot{\xi}^{c}-Q_{c} \dot{\xi}^{c}\right] u_{a} u_{b} } \\
& -\left[\frac{1}{2}(\mu-p+2 \gamma+2 \Lambda) \dot{\xi}_{c} n^{c}+v\left(\dot{\xi}^{c} u_{c}\right)+P_{c} \dot{\xi}^{c}\right] u_{a} n_{b} \\
& -\left[-\frac{1}{2}(\mu+3 p-2 \Lambda) u_{c} \stackrel{*}{\xi}^{c}-v n_{c} \stackrel{*}{\xi}^{c}-Q_{c} \stackrel{*}{\xi}^{c}\right] u_{b} n_{a} \\
& -\left[\frac{1}{2}(\mu-p-\gamma+2 \Lambda) \dot{\xi}_{d} P_{c}^{d}+Q_{d}\left(\dot{\xi}^{k} u_{k}\right) P_{c}^{d}+P_{d}\left(n_{k} \dot{\xi}^{k}\right) P_{c}^{d}+D_{d k} \dot{\xi}^{k} P_{c}^{d}\right] u_{a} P_{b}^{c} \\
& -\left[\frac{1}{2}(\mu+3 p-2 \Lambda) u_{k} \xi_{; d}^{k} P_{c}^{d}-v n_{k} \xi_{; d}^{k} P_{c}^{d}+Q_{k} \xi_{; d}^{k} P_{c}^{d}\right] u_{b} P_{a}^{c} \\
& +\left[\frac{1}{2}(\mu-p+2 \gamma+2 \Lambda)\left(\xi_{c}^{*} n^{c}\right)+v\left(u^{c} \xi_{c}^{*}\right)+P^{c} \xi_{c}^{*}\right] n_{a} n_{b} \\
& +\left[\frac{1}{2}(\mu-p-\gamma+2 \Lambda) \xi_{d}^{*} P_{c}^{d}+Q_{c}\left(u^{k} \xi_{k}^{*}\right)+P_{d} n_{k}^{*} \xi^{*} P_{c}^{d}+D_{d k} \xi^{*} P_{c}^{d}\right] n_{a} P_{b}^{c} \\
& +\left[\frac{1}{2}(\mu-p+2 \gamma+2 \Lambda) n_{k} \xi_{; d}^{k} P_{c}^{d}+v u_{c} \xi_{; d}^{c} P_{e}^{d}+P_{c} \xi_{; d}^{c} P_{e}^{d}\right] n_{b} P_{a}^{c} \\
& +\left[\frac{1}{2}(\mu-p-\gamma+2 \Lambda) \xi_{k ; r} P_{c}^{k} P_{d}^{r}+Q_{c}\left(u_{k} \xi_{; d}^{k}\right)+P_{c}\left(n_{k} \xi_{; d}^{k}\right)+D_{c k} \xi_{; d}^{k}\right] P_{a}^{c} P_{b}^{d} .
\end{aligned}
$$

Adding, we find

$$
\begin{aligned}
& R_{c(a} \xi_{; b)}^{c}=\left[-(\mu+3 p-2 \Lambda)\left(\dot{\xi}_{c} u^{c}\right)-2 v n_{c} \dot{\xi}^{c}-2 Q_{c} \dot{\xi}^{c}\right] u_{a} u_{b} \\
& -2\left[\begin{array}{c}
\left(\frac{1}{2}(\mu-p+2 \gamma+2 \Lambda) \dot{\xi}_{c} n^{c}+v\left(\dot{\xi}^{c} u_{c}\right)+P_{c} \dot{\xi}^{c}\right) \\
+\left(-\frac{1}{2}(\mu+3 p-2 \Lambda) u_{c} \stackrel{*}{\xi}^{c}-v n_{c} \stackrel{*}{\xi}^{c}-Q_{c} \stackrel{*}{\xi}^{c}\right)
\end{array}\right] u_{(a} n_{b} \\
& -2\left[\begin{array}{c}
\left(\frac{1}{2}(\mu+3 p-2 \Lambda) u_{k} \xi_{; d}^{k} P_{c}^{d}-v n_{k} \xi_{; d}^{k} P_{c}^{d}+Q_{k} \xi_{; d}^{k} P_{c}^{d}\right) \\
+\left(\begin{array}{c}
\frac{1}{2}(\mu-p-\gamma+2 \Lambda) \dot{\xi}_{d} P_{c}^{d}+Q_{d}\left(\dot{\xi}^{k} u_{k}\right) P_{c}^{d}+ \\
+P_{d}\left(n_{k} \dot{\xi}^{k}\right) P_{c}^{d}+D_{d k} \dot{\xi}^{k} P_{c}^{d}
\end{array}\right)
\end{array}\right] u_{(a} P_{b)}^{c}+ \\
& +\left[(\mu-p+2 \gamma+2 \Lambda)\left(\xi_{c}^{*} n^{c}\right)+2 v\left(u^{c} \xi_{c}^{*}\right)+2 P^{c} \xi_{c}^{*}\right] n_{a} n_{b}
\end{aligned}
$$




$$
\begin{aligned}
& +2\left[\begin{array}{c}
\left(\frac{1}{2}(\mu-p+\gamma+2 \Lambda) n_{k} \xi_{; d}^{k} P_{c}^{d}+v u_{c} \xi_{; d}^{c} P_{e}^{d}+P_{c} \xi_{; d}^{c} P_{e}^{d}\right) \\
+\left(\begin{array}{c}
\frac{1}{2}(\mu-p-\gamma+2 \Lambda) \stackrel{*}{\xi}_{d} P_{c}^{d} \\
+Q_{c}\left(u^{k} \xi_{k}^{*}\right)+P_{d} n_{k} \xi^{* k} P_{c}^{d}+D_{d k} \xi^{*^{k}} P_{c}^{d}
\end{array}\right)
\end{array}\right] n_{(a} P_{b)}^{c} \\
& +\left[\begin{array}{c}
(\mu-p-\gamma+2 \Lambda) \xi_{k ; r} P_{c}^{k} P_{d}^{r} \\
+2 Q_{c}\left(u_{k} \xi_{; d}^{k}\right)+2 P_{c}\left(n_{k} \xi_{; d}^{k}\right)+2 D_{c k} \xi_{; d}^{k}
\end{array}\right] P_{a}^{c} P_{b}^{d} .
\end{aligned}
$$

Finally, we write for the quantity $L_{\xi} R_{a b}$ in the $1+1+2$ decomposition:

$$
\begin{aligned}
& L_{\xi} R_{a b}=\left[\begin{array}{c}
\frac{1}{2}(\mu+3 p)^{\circ}+2 v \stackrel{\circ}{c}_{c} n^{c}-2 \stackrel{\circ}{Q}_{c} u^{c} \\
-(\mu+3 p-2 \Lambda)\left(\dot{\xi}_{c} u^{c}\right)-2 v n_{c} \dot{\xi}^{c}-2 Q_{c} \dot{\xi}^{c}
\end{array}\right] u_{a} u_{b} \\
& -2\left[\begin{array}{c}
-(\mu+p+\gamma) \dot{\leftrightarrow}_{c} n^{c}-\dot{v}-\grave{Q}_{c} n^{c}-P^{c} \stackrel{u}{c} \\
+\left(\frac{1}{2}(\mu-p+2 \gamma+2 \Lambda) \dot{\xi}_{c} n^{c}+v\left(\dot{\xi}^{c} u_{c}\right)+P^{c} \dot{\xi}_{c}\right) \\
+\left(-\frac{1}{2}(\mu+3 p-2 \Lambda) u_{c} \stackrel{*}{\xi}^{c}-v n_{c} \stackrel{*}{\xi}^{c}-Q_{c} \stackrel{*}{\xi}^{c}\right)
\end{array}\right] u_{(a} n_{b)} \\
& -2\left[\begin{array}{c}
-\left(\mu+p-\frac{1}{2} \gamma\right) \stackrel{\circ}{u}_{d} P_{c}^{d}-\left(v \stackrel{\circ}{d}_{d}+\stackrel{\circ}{Q}_{d}\right) P_{c}^{d}-P_{d}\left(\stackrel{\circ}{u}_{k} n^{k}\right) P_{c}^{d}-D_{k d} \stackrel{\circ}{k}^{k} P_{c}^{d} \\
+\left(\frac{1}{2}(\mu+3 p-2 \Lambda) u_{k} \xi_{; d}^{k} P_{c}^{d}-v n_{k} \xi_{; d}^{k} P_{c}^{d}+Q_{k} \xi_{; d}^{k} P_{c}^{d}\right) \\
+\left(\frac{1}{2}(\mu-p-\gamma+2 \Lambda) \dot{\xi}_{d} P_{c}^{d}+Q_{d}\left(\dot{\xi}^{k} u_{k}\right) P_{c}^{d}+P_{d}\left(n_{k} \dot{\xi}^{k}\right) P_{c}^{d}+D_{d k} \dot{\xi}^{k} P_{c}^{d}\right)
\end{array}\right] u_{(a} P_{b)}^{c} \\
& +\left[\begin{array}{c}
\left(\frac{1}{2} \mu-\frac{1}{2} p+\gamma\right)^{\circ}+2 v \stackrel{\circ}{c}_{c} n^{c}-2 P_{k} \grave{n}^{k} \\
+(\mu-p+2 \gamma+2 \Lambda)\left(\stackrel{\xi}{\xi}_{c}^{*} n^{c}\right)+2 v\left(u^{c} \xi_{c}^{*}\right)+2 P^{c} \stackrel{\xi}{\xi}_{c}^{*}
\end{array}\right] n_{a} n_{b}+ \\
& +2\left[\begin{array}{c}
v \stackrel{\circ}{u}_{d} P_{c}^{d}+\left(\stackrel{\circ}{u}_{k} n^{k}\right) Q_{d} P_{c}^{d}+\stackrel{\circ}{P}_{d} P_{c}^{d}+\frac{3}{2} \gamma \stackrel{\circ}{n}_{c}-D_{d c} \stackrel{\circ}{n}^{d} \\
+\left(\frac{1}{2}(\mu-p+2 \gamma+2 \Lambda) n_{k} \xi_{; d}^{k} P_{c}^{d}+v u_{c} \xi_{; d}^{c} P_{e}^{d}+P_{c} \xi_{; d}^{c} P_{e}^{d}\right) \\
+\left(\frac{1}{2}(\mu-p-\gamma+2 \Lambda) \stackrel{*}{\xi}_{d} P_{c}^{d}+Q_{c}\left(u^{k}{ }^{*} \xi_{k}\right)+P_{d} n_{k} \stackrel{*}{\xi}^{k} P_{c}^{d}+D_{d k} \stackrel{*}{\xi}^{k} P_{c}^{d}\right)
\end{array}\right] n_{(a} P_{b)}^{c} \\
& +\left[\begin{array}{c}
\left.\frac{1}{2}(\mu-p-\gamma)^{\circ} P_{c d}+2 Q_{(k} \stackrel{\circ}{u}\right)_{r} P_{c}^{k} P_{d}^{r}+2 P_{(c} \stackrel{\circ}{n}_{d)}+\stackrel{\circ}{D}_{k r} P_{c}^{k} P_{d}^{r} \\
(\mu-p-\gamma+2 \Lambda) \xi_{k ; r} P_{c}^{k} P_{d}^{r}+2 Q_{c}\left(u_{k} \xi_{; d}^{k}\right)+2 P_{c}\left(n_{k} \xi_{; d}^{k}\right)+2 D_{c k} \xi_{; d}^{k}
\end{array}\right] P_{a}^{c} P_{b}^{d} .
\end{aligned}
$$

If we replace $\xi^{a}=-\lambda u^{a}+\phi n^{a}$, we will find the complete answer in the final $1+1+2$ form. In the following, we work with the particular cases $\xi^{a}=-\lambda u^{a}$ and $\xi^{a}=\phi n^{a}$.

17.1.1 The case $\xi^{a}=-\lambda u^{a}$

When $\xi^{a}=-\lambda u^{a}$ we find

$$
\begin{aligned}
L_{\left(-\lambda u^{a}\right)} R_{a b}= & -\lambda\left[\frac{1}{2}(\mu+3 p)^{\cdot}+(\mu+3 p-2 \Lambda)(\ln \lambda)^{\cdot}\right] u_{a} u_{b} \\
& +\lambda\left[\begin{array}{c}
-(\mu+3 p-2 \Lambda)\left(\dot{u}_{c} n^{c}-(\ln \lambda)^{*}\right)-2 v(\ln \lambda)^{\cdot} \\
-2 \dot{v}+2 Q^{c} N_{c}-2 v\left(\sigma_{d c} n^{c} n^{d}+\frac{1}{3} \theta\right)
\end{array}\right] u_{(a} n_{b)}
\end{aligned}
$$




$$
\begin{aligned}
& +\lambda\left[\begin{array}{c}
-(\mu+3 p-2 \Lambda)\left[\dot{u}_{c}-(\ln \lambda)_{, c}\right]-2 Q_{c}(\ln \lambda)^{\cdot} \\
-2 v N_{c}-4 v \sigma_{d c} n^{d}-2 \dot{Q}_{c}-2 Q_{d}\left(\omega_{. c}^{d}+\sigma_{. c}^{d}+\frac{1}{3} \theta h_{c}^{d}\right)
\end{array}\right] u_{(a} P_{b)}^{c} \\
& -\lambda\left[\begin{array}{c}
\frac{1}{2}(\mu-p+2 \gamma)^{\cdot}+(\mu-p+2 \gamma+2 \Lambda)\left(\sigma_{c d} n^{c} n^{d}+\frac{1}{3} \theta\right) \\
-2 P_{c} N^{c}+2 v\left[\dot{u}_{d} n^{d}-(\ln \lambda)^{*}\right]
\end{array}\right] n_{a} n_{b} \\
& -\lambda\left[\begin{array}{c}
2(\mu-p+2 \gamma+2 \Lambda) \sigma_{c d} n^{d}+\frac{6}{2} \gamma N_{c}+2 \dot{P}_{c}-2 D_{c d} N^{d}+\frac{4}{3} \theta P_{c} \\
+2 Q_{c}\left[\dot{u}_{d} n^{d}-(\ln \lambda)^{*}\right]+2 v\left[\dot{u}_{c}-(\ln \lambda)_{, c}\right] \\
+2 P_{c}\left(\sigma_{d e} n^{d} n^{e}\right)+2 P_{d}\left(\sigma_{. c}^{d}+\omega_{. c}^{d}\right)
\end{array}\right] n_{(a} P_{b)}^{d}+ \\
& -\lambda\left[\begin{array}{c}
\frac{1}{2}(\mu-p-\gamma)^{\cdot} P_{c d}+(\mu-p-\gamma+2 \Lambda)\left(\sigma_{c d}-\frac{1}{3} \theta P_{c d}\right) \\
+2 P_{(c} P_{d)}^{e}\left[N_{e}+\left(\sigma_{e f}+\omega_{e f}\right) n^{f}\right]+\dot{D}_{c d}+\frac{2}{3} \theta D_{c d} \\
+2 Q_{(c}\left[\dot{u}_{d)}-(\ln \lambda)_{; d)}\right]+2 P_{(c}\left(\omega_{. d)}^{e}+\sigma_{. d)}^{e}\right) n_{e}+2 D_{e(c}\left(\omega_{. d)}^{e}+\sigma_{. d)}^{e}\right)
\end{array}\right] P_{a}^{c} P_{b}^{d} .
\end{aligned}
$$
part:

The last term can be decomposed into trace and a trace-free part. Contracting with $P^{a b}$, we have the trace

$$
I=-\frac{\lambda}{2}\left[\begin{array}{c}
(\mu-p-\gamma)^{\cdot}-(\mu-p-\gamma+2 \Lambda)\left(\sigma_{c d} n^{c} n^{d}-\frac{2}{3} \theta\right) \\
+2 P^{e}\left[N_{e}+\sigma_{e f} n^{f}\right]+2 Q^{e}\left[\dot{u}_{e}-(\ln \lambda)_{; e)}\right]+2 D_{e c} \sigma^{e c}
\end{array}\right]
$$

and the trace free part:

$$
H_{a b}=-\lambda\left[\begin{array}{c}
(\mu-p-\gamma+2 \Lambda) \sigma_{c d}+2 P_{(c} P_{d)}^{e}\left[N_{e}+\left(\sigma_{e f}+\omega_{e f}\right) n^{f}\right]-P^{e}\left[N_{e}+\sigma_{e f} n^{f}\right] P_{c d}+ \\
+\dot{D}_{c d}+\frac{2}{3} \theta D_{c d}+2 Q_{(c}\left[\dot{u}_{d)}-(\ln \lambda)_{; d)}\right]-Q^{e}\left[\dot{u}_{e}-(\ln \lambda)_{e)}\right] P_{c d}+ \\
+2 P_{(c}\left(\omega_{. d)}^{e}+\sigma_{. d)}^{e}\right) n_{e}+2 D_{e(c}\left(\omega_{. d)}^{e}+\sigma_{. d)}^{e}\right)-\left(D_{e c} \sigma^{e c}\right) P_{c d}
\end{array}\right]
$$

17.1.2 The case $\xi^{a}=\phi n^{a}$

When $\xi^{a}=\phi n^{a}$, we calculate

$$
\begin{aligned}
L_{\left(\phi n^{a}\right)} R_{a b}= & \phi\left[\begin{array}{c}
\frac{1}{2}(\mu+3 p)^{*}+(\mu+3 p-2 \Lambda)\left(\dot{u}^{c} n_{c}\right) \\
-2 v\left[(\ln \phi)^{\cdot}-\sigma_{c d} n^{c} n^{d}+\frac{1}{3} \theta\right]-2 Q_{c} N^{c}
\end{array}\right] u_{a} u_{b} \\
& -2 \phi\left[\begin{array}{c}
\frac{1}{2}(\mu-p+2 \Lambda+2 \gamma)\left[(\ln \phi)^{\cdot}-\left(\sigma_{c d} n^{c} n^{d}+\frac{1}{3} \theta\right)\right] \\
+P^{c} N_{c}-\stackrel{*}{v}-v\left[(\ln \phi)^{*}+\dot{u}^{c} n_{c}\right]
\end{array}\right] u_{(a} n_{b)} \\
& -2 \phi\left[\begin{array}{c}
\frac{1}{2}(\mu-p-\gamma+2 \Lambda) N_{c}+(\mu+3 p-2 \Lambda) \omega_{d c} n^{d} \\
+P_{c}\left[(\ln \phi)^{\cdot}-\left(\sigma_{e f} n^{e} n^{f}+\frac{1}{3} \theta\right)\right]+D_{d c} N^{d}-v\left(\stackrel{*}{n}_{c}+(\ln \phi)_{; c}\right) \\
-Q_{c}\left(\dot{u}^{d} n_{d}\right)-\stackrel{*}{Q}_{d} P_{c}^{d}-Q_{r}\left(R_{. d}^{r}+S_{d d}^{r}+\frac{1}{2} E P_{d}^{r}\right)
\end{array}\right] u_{(a} P_{b)}^{c}
\end{aligned}
$$




$$
\begin{gathered}
+\phi\left[\frac{1}{2}(\mu-p+2 \gamma)^{*}+(\mu-p+2 \Lambda+2 \gamma)(\ln \phi)^{*}\right] n_{a} n_{b} \\
+2 \phi\left[\begin{array}{c}
*_{c}+\frac{1}{2}(\mu-p+2 \gamma+2 \Lambda)\left({ }^{*} n_{c}+(\ln \phi)_{; c}\right)-2 v \omega_{d c} n^{d}+ \\
+P_{r}\left[\left(R_{. c)}^{r}+S_{. c)}^{r}+\frac{1}{2} E P_{c)}^{r}\right)+(\ln \phi)^{*} P_{c}^{r}\right]
\end{array}\right] n_{(a} P_{b)}^{c} \\
+\phi\left[\begin{array}{c}
\frac{1}{2}(\mu-p-\gamma)^{*} P_{c d}+(\mu-p-\gamma+2 \Lambda)\left(S_{c d}+\frac{1}{2} P_{c d} E\right)+ \\
+{ }^{*} D_{c d}+2 D_{r(c}\left(R_{. d)}^{r}+S_{. d)}^{r}+\frac{1}{2} E P_{d)}^{r}\right) \\
+4 Q_{(c} \omega_{d) r} n^{r}+2 P_{(c} P_{d)}^{e}\left({ }^{*}{ }_{e}+(\ln \phi)_{; e}\right)
\end{array}\right] P_{a}^{c} P_{b}^{d} .
\end{gathered}
$$

The trace and the trace free parts of the last term are

$$
\begin{gathered}
I=\frac{\phi}{2}\left[\begin{array}{c}
(\mu-p-\gamma)^{*}+(\mu-p-\gamma+2 \Lambda) E+2 D_{r c} S^{r c} \\
+4 Q^{d} \omega_{d r} n^{r}+2 P^{e}\left({\stackrel{*}{(}{ }_{e}}^{*}(\ln \phi)_{; e}\right)
\end{array}\right] \\
H_{a b}=+\phi\left[\begin{array}{c}
(\mu-p-\gamma+2 \Lambda) S_{c d}+\stackrel{*}{D}_{c d}+2 D_{r(c}\left(R_{. d)}^{r}+S_{. d)}^{r}+\frac{1}{2} E P_{d)}^{r}\right) \\
-\left(2 D_{r c} J^{r c}\right) P_{c d}+4 Q_{(c} \omega_{d) r} n^{r}-2\left(Q^{d} \omega_{d r} n^{r}\right) P_{c d} \\
+2 P_{(c} P_{d)}^{e}\left(n_{e}^{*}+(\ln \phi)_{; e}\right)-P^{e}\left(n_{e}+(\ln \phi)_{; e}\right) P_{c d}
\end{array}\right] .
\end{gathered}
$$

These general results can be used to study the kinematics and he dynamics of all spacetime models for all types of observers, all types of symmetries and for general matter fields.

Acknowledgements AP acknowledges the financial support of FONDECYT Grant no. 3160121.

Open Access This article is distributed under the terms of the Creative Commons Attribution 4.0 International License (http:// creativecommons.org/licenses/by/4.0/), which permits unrestricted use, distribution, and reproduction in any medium, provided you give appropriate credit to the original author(s) and the source, provide a link to the Creative Commons license, and indicate if changes were made.

\section{References}

1. Anderson, J.L.: Principles of Relativity Physics. Academic Press, New York (1973)

2. Baysal, H.; Yilmaz, I.: Spacelike Ricci inheritance vectors in a model of string cloud and string fluid stress tensor. Class. Quantum Grav. 19, 6435 (2002)

3. Baysal, H.; Camci, U.; Tarhan, I.; Yilmaz, I.: Ricci collineations of the Bianchi types I and III, and Kantowski-Sachs spacetimes. IJMPD 11, 463 (2002)

4. Camci, U.: Conformal collineations and Ricci inheritance symmetry in string cloud and string fluids. IJMPD 11, 353 (2002)

5. Dirac, P.: General Theory of Relativity. Princeton University Press, Wiley, New York (1975)

6. Einstein, A.: Grundgedanken der allgemeinen Relativitätstheorie und Anwendung dieser Theorie in der Astronomie, 315th edn. Preussische Akademie der Wissenschaften, Sitzungsberichte (1915)

7. Einstein, A.: Zur allgemeinen Relativitätstheorie (On the General Theory of Relativity), 778th edn. Preussische Akademie der Wissenschaften, Sitzungsberichte (1915)

8. Einstein, A.: Erklärung der Perihelbewegung des Merkur aus der allgemeinen Relativitätstheorie, 831st edn. Preussische Akademie der Wissenschaften, Sitzungsberichte (1915)

9. Einstein, A.: Feldgleichungen der Gravitation, 844th edn. Preussische Akademie der Wissenschaften, Sitzungsberichte (1915)

10. Ellis, G.F.R.: Dynamics of pressure-free matter in general relativity. J. Math. Phys. 8, 1171 (1967)

11. Ellis, G.F.R.; Elst, H.V.: Cosmological models. In: Cargèse Lectures. arXiv:gr-qc/9812046

12. Herrera, L.; Jimenez, J.; Leal, L.; Ponce de Leon, J.; Esculpi, M.; Galina, V.: Anisotropic fluids and conformal motions in general relativity. J. Math. Phys. 25, 3274 (1984)

13. Katzin, G.H.; Levine, J.; Davis, W.R.: Curvature collineations: a fundamental symmetry property of the space-times of general relativity defined by the vanishing Lie derivative of the Riemann curvature tensor. J. Math. Phys. 10, 617 (1969)

14. Krasiński, A.: Inhomogeneous Cosmological Models. Cambridge University Press, New York (2006)

15. Letelier, P.: Anisotropic fluids with two-perfect-fluid components. Phys. Rev. D 22, 807 (1980)

16. Letelier, P.: Inheriting conformal and special conformal Killing vectors in string cosmology. Nuovo Cim. B63, 519 (1981)

17. Letelier, P.: String cosmologies. Phys. Rev. D 28, 2414 (1983) 
18. Lie, S.: Theorie der Transformationsgruppen I. B. G. Teubner, Leipzig (1888)

19. Lund, F.; Regge, T.: Unified approach to strings and vortices with soliton solutions. Phys. Rev. D 14, 1524 (1976)

20. Maartens, R.; Mason, D.P.; Tsamparlis, M.: Kinematic and dynamic properties of conformal Killing vectors in anisotropic fluids. J. Math. Phys. 27, 2987 (1986)

21. Nakamura, M.: Geometry, Topology and Physics. Taylor \& Francis Group, Florida (2003)

22. Penrose, R.: A spinor approach to general relativity. Ann. Phys. 10, 171 (1960)

23. Ray, D.: Solutions of coupled Einstein-SO(3) gauge field equation. Phys. Rev. D 18, 3879 (1978)

24. Saridakis, E.; Tsamparlis, M.: Symmetry inheritance of conformal Killing vectors. J. Math. Phys. 32, 1541 (1991)

25. Sharif, M.; Sheikh, U.: Timelike and spacelike matter inheritance vectors in specific forms of energy-momentum tensor. IJMPA 21, 3213 (2006)

26. Stephani, H.; Kramer, D.; MacCallum, M.; Hoenselaers, C.; Herlt, E.: Exact Solutions of Einstein's Field Equations. Cambridge University Press, Cambridge (2003)

27. Stewart, J.M.; Ellis, G.F.R.: Solutions of Einstein's equations for a fluid which exhibit local rotational symmetry. J. Math. Phys. 9, 1072 (1968)

28. Tsamparlis, M.: Geometrization of a general collineation. J. Math. Phys. 33, 1472 (1992)

29. Tsamparlis, M.: Geometrization of a general collineation. J. Math. Phys. 33, 1472-1479 (1992)

30. Tsamparlis, M.: General symmetries of a string fluid space-time. Gen. Relat. Grav. 38, 311 (2006)

31. Tsamparlis, M.; Mitsopoulos, A.; Paliathanasis, A.: Symmetries of spacetimes embedded with an electromagnetic string fluid. Gen. Relat. Grav. 51, 6 (2019)

32. Yano, K.: The Theory of Lie Derivatives and Its Applications. North Holland, Amsterdam (1956)

33. Yavuz, I.; Yilmaz, I.: Topological defect solutions in the spherically symmetric space-time admitting conformal motion. Gen. Relat. Grav. 9, 1295 (1997)

34. Yilmaz, I.: Timelike and spacelike Ricci collineation vectors in string cosmology. IJMPD 10, 681 (2001)

Publisher's Note Springer Nature remains neutral with regard to jurisdictional claims in published maps and institutional affiliations. 